\title{
Tank Farm Surveillance and Waste Status Summary Report for December 1992
}

B. M. Hanion

Date Published

February 1993

Prepared for the U.S. Department of Energy Office of Environmental Restoration and Waste Management

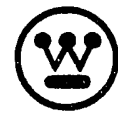

Westinghouse

Hanford Company Richland, Washington 99352

Hanford Operations and Engineering Contractor for the

U.S. Department of Energy under Contract DE-AC06-87RL10930

DISTRIBUTION OF THIS DOCUMENT IS UNLIMITED

Approved for Public Release 
WHC-EP-0182-57

APPROVAL

Prepared by: $\frac{\text { P.Yh A Anlow }}{\substack{\text { B.M.Hanlon } \\ \text { Engineer }}} \frac{1-26-93}{\text { Date }}$

Approved by: $\frac{1 \text { tes }}{\begin{array}{l}\text { G.T. Frater } \\ \text { Manager, Surveillance } \\ \text { and Data Acquisition }\end{array}} \frac{1-29-93}{\text { Date }}$ 


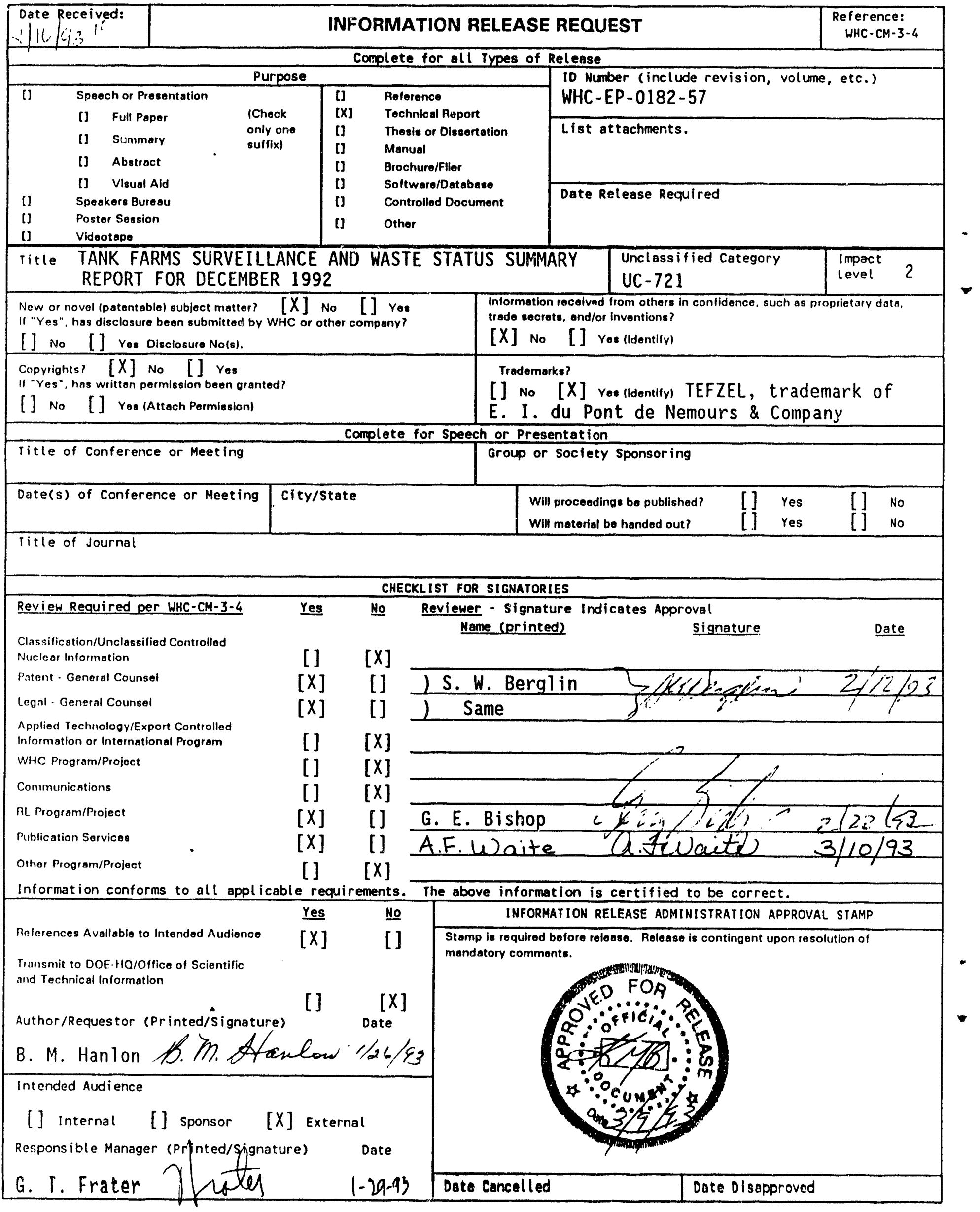


WHC,-EP-0182-57

\title{
TANK FARM SURVEILLANCE AND WASTE STATUS SUMMARY REPORT FOR DECEMBER 1992
}

\author{
B. M. Hanlon
}

\section{ABSTRACT}

This report is the official inventory for radioactive waste stored in underground tanks in the 200 Areas at the Hanford Site. Data that depict the status of stored radioactive waste and tank vessel integrity are contained within the report. This report provides data on each of the existing 177 large underground waste storage tanks and 49 smaller catch tanks and special surveillance facilities, and supplemental information regarding tank surveillance anomalies and ongoing investigations. This report is intended to meet the requirement of U.S. Department of Energy-Richland Operations office Order 5820.2A, Chapter I, Section 3.e. (3) (DOE-RL, 1990, Radioactive Waste Management, U. S. Department of Energy-Richland Operation Office, Richland, Washington) requiring the reporting of waste inventories and space utilization for Hanford Tank Farm Tanks. 
WHC-EP-0182-57

This page intentionally left blank. 


\section{CONTENTS}

SUMMARY

TANK STATUS

TANK INVESTIGATIONS .......................... 1

HIGHLIGHTS . . . . . . . . . . . . . . . . . . . . . . . 3

Appendixes:

A. TANK AND EQUIPMENT CODE AND STATUS DEFINITIONS . . . . . . . . . . . A-1

Tank and Equipment Code/Status Definitions . . . . . . . . . . A-3

B. TANK FARM CONFIGURATION, STATUS AND FACILITY CHARTS . . . . . . . . . . B-1

1 High-Level Waste Tank Configuration . . . . . . . . . . . . . . B-3

2 Double-Shell Tank Instrumentation Configuration . . . . . . . B-4

3 Single-Shell Tank Instrumentation Configuration . . . . . . . B-5

4 Double-Shell Tank Status . . . . . . . . . . . . . . B-6

5200 E Single-Shell Tank Status .............. . B-7

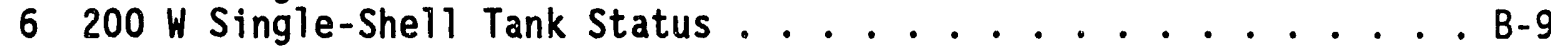

7 Tank Farm Facilities Quick Reference . . . . . . . . . . . . . B-11

8 Hanford Tank Farms Facilities Chart . . . . . . . . . . . . B-12

C. MONTHLY SUMMARY ........................... C-1

1 Monthly Summary .....................C-3

2 Tank Use Summary . . . . . . . . . . . . . . . . . C-4

3 Inventory Summary by Tank Farm . . . . . . . . . . . . . C -5

4 Inventory and Status by Tank - Double-Shell Tanks . . . . . . . C-6

5 Inventory and Status by Tank - Single-She11 Tanks . . . . . . . . C-9

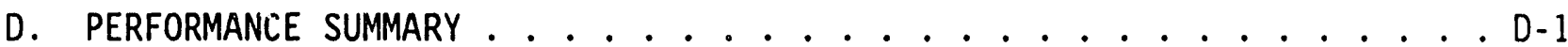

1 Performance Summary . . . . . . . . . . . . . . . D-3

E. LIQUID STATUS AND PUMPABLE LIQUID REMAINING IN TANKS . . . . . . . . . E-1

1 Liquid Status and Pumpable Liquid Remaining in Tanks . . . . . . . E-3

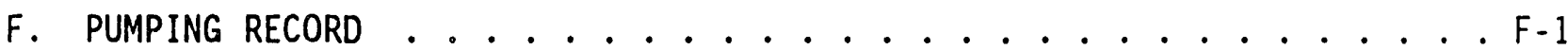

1 Pumping Record ..................... . F-3

G. CATCH TANKS AND SPECIAL SURVEILLANCE FACILITIES . . . . . . . . . . . . G-1

1 East and West Area Catch Tanks and Special

Surveillance Facilities (Active) . . . . . . . . . . . . . G-3

2 East Area Catch Tanks and Special Surveillance

Facilities (Inactive) .................. . G-4

3 West Area Catch Tanks and Special Surveiliance

Facilities (Inactive) ................. G-5

H. LEAK VOLUME ESTIMATES ....................... H-1

1 Single-Shell Tank Leak Volume Estimates . . . . . . . . . . . . H-3 
WHC-EP-0182-57

This page intentionally left blank.

viii 


\section{LIST OF FIGURES}

1 Current Status and Contingency Space for the 242-A

Evaporator Restart ............... . 25

2 Master Core Sampling Schedule .............. 26

\section{LIST OF TABLES}

1 Watch List Tanks ................. . . . 7

2 Tanks Containing $>1,000$ gram mole of Ferrocyanide (Watch List Tanks) . . . . . . . . . 8

3 Tanks with Potential for Hydrogen or Flammable Gas Accumulation Above the Flammability Limit (Watch List Tanks) . . . . . . . 13

4 Tanks Containing Concentrations of Organic Salts $>3 \%$ Weight TOC (Watch List Tanks) . . . . . . . 14

5 Single-Shell Tanks with High Heat Loads $(>40,000 \mathrm{Btu} / \mathrm{hr}) \ldots 15$

6 Double-Shell Tank Waste Type and Space Allocation . . . . . . 18

7 Automatic Food Instrument Company (FIC) Gauges Out of Service . . 19

8 Single-Shell Tanks Monitoring Compliance Status .......... 20

9 Double-Shell Tanks Monitoring Compliance Status . . . . . . . . . . 24

\begin{tabular}{|c|c|c|}
\hline \multicolumn{3}{|c|}{ METRIC CONVERSION CHART } \\
\hline 1 inch & $=$ & 2.54 centimeters \\
\hline 1 foot & $=$ & 30.48 centimeters \\
\hline 1 gallon & $=$ & 3.80 liters \\
\hline 1 ton & $=$ & 0.90 metric tons \\
\hline \multicolumn{3}{|c|}{${ }^{\circ} \mathrm{F}=\left(\frac{9}{5}{ }^{\circ} \mathrm{C}\right)+32$} \\
\hline \multicolumn{3}{|c|}{$\begin{array}{c}1 \mathrm{Btu} / \mathrm{h}=2.930711 \mathrm{E}-01 \text { watts } \\
\text { (International Table) }\end{array}$} \\
\hline
\end{tabular}


WHC-EP-0182-57

This page intentionally left blank 
WHC-EP-0182-57

\section{TANK FARM SURVEILLANCE AND WASTE STATUS SUMMARY REPORT FOR DECEMBER 1992 \\ SUMMARY}

Note: Changes from the previous month are in bold print.

\section{TANK STATUS}

\begin{tabular}{|l|r|c|}
\hline \multicolumn{1}{|c|}{ Category } & Quantity & Date of Last Change \\
\hline In-Service Tanks & & $10 / 86$ \\
\hline Out-of-Service Tanks & 28 double-shell & $07 / 88$ \\
\hline Assumed-Leaker Tanks & 149 single-shell & $10 / 92$ \\
\hline Interim-Stabilized Tanks & 67 single-shell & $09 / 90$ \\
\hline Interim-Isolated Tanks & 105 single-shell & $09 / 91$ \\
\hline
\end{tabular}

a All 149 single-shell tanks were removed from service (i.e., no longer authorized to receive waste) as of November 21, 1980.

b the 105 tanks classified as interim stabilized, 58 are listed as assumed leakers. The total of 105 interim stabilized tanks includes eight tanks that do not meet current established supernatant and interstitial liquid stabilization criteria: 104-B, 107-B, 110-B, 111-B, 110-BX, 102-T, 112-T, and 110-U.

c Six double-shell tanks listed as "in service" are currently not receiving waste because of inclusion on the Hydrogen Watch List and are thus prohibited from receiving waste in accordance with "Safety Measures for Waste Tanks at Hanford Nuclear Reservation," Section 3137 of the National Defense Authorization Act for Fiscal Year 1991, November 5, 1990, Public Law 101-510.

' of the 48 single-shell tanks on Watch Lists, 21 have been Interim Stabilized.

e of the 48 single-shell tanks on Watch Lists, 20 have been Interim Isolated.

of the 67 assumed ieaker tanks, nine have not yet been interim stabilized. See Appendix $\mathrm{H}$ for more details.

\section{TANK INVESTIGATIONS}

Tank 241-SY-101. The surface level within this tank continues to fluctuate. The surface level increase/decrease phenomena has been observed since 1981, and is attributed to the slow buildup and relatively fast release of gas within the waste. An investigation into solutions to this slurry growth problem is ongoing. Multiple Event Fact Sheets, a Critique Report, Occurrence Reports, Discrepancy Reports, and Unusual Occurrence Reports have been issued. The Food Instrument Company (FIC) surface level monitor showed an increase from 406.20 inches on December 1 to 408.70 inches by December 31,1992 . The manual tape increased from 410.25 to 414.75 inches by December 26 , and decreased to 409.00 inches by December 31, 1992 (a new manual tape was installed on October 5, 1992, which is 10-7/8 inches longer than the previous manual tape). The radar gauge reading was 409.23 inches December 1 and 410.93 inches on December 31,1992 . The surface level measurements for the radar gauge, FIC, and manual tape are different due to the location of the 
WHC-EP-0182-57

measurement devices, irregular surface, and the different method by which the level is obtained.

Tank 241-SX-103. The level showed erratic increases/decreases from 1981 to March 1983. The level showed a decreasing trend from 247.90 inches in March 1983 to 242.10 inches on February 3, 1992. The FIC was out of service from February 10 to September 21, 1992. Following FIC repair on September 21, the level was reading 240.00 inches, increased to 241.00 inches on November 28 , and was out of service November 29 and 30, 1992. The surface level reading was 241.60 inches after FIC repair on December 1,1992 , and 241.50 inches on December 31 , 1992. The Interstitial Liquid Level (ILL) has remained stable at 20.5 feet $(-0.1$ feet from the established baseline since March 1, 1991), and was last scanned November 27, 1992. An evaporation study was completed by Waste Characterization Analysis, and concluded that the observed rate of liquid level decrease is within the range expected from evaporation. A small leak could easily be masked by modeling uncertainties. It has been determined by Surveillance and Data Acquisition that the observed decrease in the surface level measurement is attributed to the FIC plummet contacting a dry irregular surface in a depression (based on in-tank photographs taken December 17 , 1987). The FIC will be placed from the automatic to the intrusion mode of operation. Liquid observation well (LOW) scan data will be the primary means of leak detection. The LOW monitoring frequency has been increased from quarterly to monthly. Drywell scan data remain stable. This tank will not appear on future reports unless new anomalies are found in the surveillance data.

Tank 241-SX-105. An Interstitial Liquid Level (ILL) baseline of 21.2 feet was established for Tank 105-SX Liquid Observation Well on August 27, 1986. The current scan of December 30, 1992, shows a 0.3 -foot decrease below baseline which is at the decrease criteria. An evaporation study was to have been performed by Waste Characterization Analysis to determine if the decrease could be attributed to evaporation. An evaporation study cannot be performed for tank 105-SX because of the complete solid surface of the tank. At the present time, there is no computer model available for determining evaporation rates for tanks with a $100 \%$ solid surface. LOW monitoring has been increased from quarterly to every two weeks. In-tank photographs taken June 15, 1988, show a dry cracked, uneven surface. The FIC is in the intrusion mode of operation, and the FIC plummet is suspended over solids. Drywell scan data remain stable. Work is in progress to repair the radiation detection laterals underneath this tank to provide improved leak detection. A tank leak assessment team is being formed to review this tank in January 1993.

Tank 241-SX-106. The surface level measurement baseline was adjusted on April 3, 1991, for an increase attributed to a steam coil leak that occurred March 13, 1991 (reference Occurrence Report WHC-91-0206-TFARM). The level increased an additional 1.20 inches by May 17, 1991, after baseline change. There has been a decreasing trend in the surface level measurement from 209.90 inches on May 17, 1991, to 206.00 inches by December 31, 1992. The decrease criteria is 5.00 inches from reference baseline of 208.70 inches. The FIC plummet is contacting solids. In-tank photographs taken June 1, 1989, show what appears to be a floating solid (foam-like) surface. The LOW ILL increased from 16.3 feet (from reference basel ine) to 16.6 feet by March 19 , 1991, and remained at 16.6 feet to october 24, 1991. The ILL decreased to 16.3 feet $(-0.3$ feet) by 0 ctober 8,1992 , and has remained at 16.3 feet during December 1992. An evaporation study has been completed by Waste 
Characterization Analysis. The analysis indicated that observed liquid level decreases could be attributed to evaporation and are within the range expected from evaporation. A small leak could easily be lasked by modeling uncertainties. LOW monitoring has been increased to very two weeks. Drywells associated with 106-SX are stable. This tank continues to be under investigation.

\section{Potential or Assumed Leaks:}

Tank 241-T-101. A surface level decrease of 2.60 inches from the previously established liquid level of 44.20 inches was noted on September 18, 1992 , following maintenance on the 101-T Level Indicator (FIC). Because the FIC had been operating only sporadically since December 1991, and no reliable surface level data was obtained between December 1991 and July 1992, the decrease was assumed to be due to the faulty FIC, and no occurrence reporting action was taken during that time. Occurrence Report RL-WHC-TANKFARM-1992-0073 was issued October 1, 1992, and Tank 101-T was declared an assumed leaker on october 4, 1992. The FIC surface level measurement was 41.50 inches on October 31,1992 , and increased to 43.80 inches after a reference elevation check was performed by instrument technicians on November 14, 1992. The FIC surface level measurement is reading 43.55 inches on December 31,1992 . A manual tape surface level measurement device was installed on December 18 , 1992. The measurement from this device has been steady at 40.5 inches. Drywell scan data was reviewed and is stable. Drywell monitoring (gross gamma) frequencies have been increased to weekly. Plans are underway to begin pumping this tank by March 15, 1993.

\section{Potential or Assumed Intrusions:}

The following tanks have been reported for potential in-leakage (intrusions) from known/unknown sources.

Tank 241-B-202. A steady increase in the surface level measurement has been observed since December 1984. The manual tape pencil plummet is contacting liquid. When the quarterly reading was obtained on October 6, 1992, the level was recorded as 144.75 inches, thus exceeding the 2.00 -inch increase criteria from the established baseline of 142.50 inches The surface level measurement was rechecked on October 9, 1992, (145.50 inches) and October 13 (145.00 inches), verifying the increase and that the criteria had been exceeded. The monitoring frequency has been increased from quarterly to monthly. The reading for December 29, 1992, was 144.75 inches. Tank 202-B has been added to the Alert List and will be reported in accordance with applicable reporting requirements.

Tank 241-S-107. A slow increase in the surface level has been observed since May 1987. The surface level measurement increased 1.10 inches in September 1991, when water was added to the tank in order to install a saltwell screen. The reference baseline was adjusted to reflect this water addition. On August 3 , 1992, the surface level measurement was 144.50 inches, thus exceeding the 2.00 -inch increase criteria from the established baseline of 142.40 inches. The FIC was reported out of service on August 17, 1992. After the FIC was repaired on September 21, the surface level measurement was 144.20 inches, which is within criteria limits. The reading remained at the 144.30-inch level to December 28, 1992. This tank will remain under close surveillance for additional unexplained surface level increases. 
Tank 241-TX-115. The LOW scans revealed an ILL increase over baseline in excess of the established 0.4-foot increase criteria in May 1987. Comparison of past and present in-tank photographs show no significant change in surface conditions or obvious evidence of intrusion. Environmental Protection Deviation Report \#88-08 was issued on May 18, 1988. Event Fact Sheet \#TF-EFS80-140 was issued January 9, 1990. The ILL showed an additional increase of 0.4 feet on March 1, 1991. The increase was verified on March 11, 1991 . These LOWs are monitored quarterly, alternating every six weeks with the neutron and gamma robes. No further increase of the ILL has been observed since March 1, 199\%. The LOW was last scanned with the neutron probe on October 23, 1992.

Tank 241-TY-102. On November 8, 1992, the automatic FIC surface level measurement of 32.10 inches exceeded the 0.50 -inch increase criteria from the reference baseline of 31.40 inches. Discrepancy Report S\&DA-92-489 was issued November 9, 1992. The tank has a history of icicle buildup on the FIC plummet and iritrusions. A request was made on November 16 for Tank Farm Operations to flush the FIC plummet. Work Order 2W-92-01155 was written to perform the flush, scheduled for December 3, 1992. The reference elevation of the FIC was verified on December 17, 1992. The FIC plummet was clean.

\section{HIGHLIGHTS}

\section{Criticality Safety Issues}

On April 30, 1992, an Unreviewed Safety Question (USQ) concerning criticality safety issues in the Tank Farms was declared to be a reportable event. Unusual Occurrence report RL-WHC-TANKFARM-1992-0037 was issued. A prohibition was placed on all waste transfers into and between the tank farm facilities which is negatively affecting various Hanford programs. On September 1, 1992, the approved Justification For Continued Operations (JCO) was received. This establishes the limitations for all tanks receiving transfers and also excludes any interim stabilization of single-shell tanks until further evaluations are completed and approved by DOE-HQ. On December 15, 1992, the approved JCO was issued as WHC-SD-WM-JCO-001, "Justification for Continued Operations of Hanford High Level Waste Tanks Resulting From the Criticality USQ, 492-CRITSAS." An amendment request to this JCO to pump and stabilize Tank 241-T-101 has been sent to DOE-HQ for approval. The resolution of the USQ is scheduled for summer 1993.

\section{Saltwell Pumping}

Interim stabilization has been delayed because of the criticality safety issues which suspended waste transfers into and between tank farm facilities. The approved Justification For Continued Operations (JCO) excludes any stabilization of single-shell tanks until further evaluations are completed and approved by DOE-HQ. The resolution of the USQ is scheduled for summer 1993. 


\section{Occurrence Reports}

RL-WHC-TANKFARM-1992-0090 (OFF-NORMAL) - 241-AN-107 LEAK DETECTION ANOMALY -- NONE OF THE OTHER LEAK DETECTION METHODS GIVE ANY INDICATION OF A LEAK (10 Day Report)

The annulus continuous air monitor (CAM) for the 107-AN has given periodic higher than normal readings since 1985. The most recent notable radiation spike occurred on July 21, 1992, when the level was recorded at 120,000 disintegrations per minute $(\mathrm{dpm})$. The laboratory analysis of the filter paper determined that the radionuclides were from the Radon daughter product chain.

The possibility of a primary tank leak has been historically evaluated and ruled out due to the lack of long-lived radionuclides on the CAM filters. Additionally, none of the other leak detection methods give any indication of a leak. There are other tanks that demonstrate similar trends to a lesser magnitude/frequency. An investigation is continuing.

RL-WHC-TANKFARM-1992-0091 (OFF-NORMAL) - MERCURY SPILL (UP TO APPROXIMATELY 3 FLUID OUNCES) DISCOVERED IN THE CONDENSER ROOM OF THE 242-A EVAPORATOR; DATE AND SOURCE OF SPILL UNDETERMINED (10 Day Report)

On December 11, 1992, while performing housekeeping activities in the 242-A Condenser Room, a small amount of mercury was discovered on the second, third, and fourth floor gratings. The liquid was visually identified as liquid metallic mercury. The spill may have been from a manometer previously used for instrument calibrations although the exact source of the mercury is unknown. The manometer was removed from the condenser room by operations personnel qualified to work in a hazardous waste site prior to the discovery of the spill. The condenser room is a five-story surface contamination area with a steel grating floor on the top four levels.

The spill apparently occurred on the fourth level. The mercury then cascaded through four levels of grating which resulted in the liquid breaking up into tiny droplets and bouncing throughout the room. Although the total amount was small, the mercury was dispersed over a very wide area, The amount contained in the manometer tube, identified as the most likely source, is three fluid ounces. The mercury escaped early detection because it was widely dispersed in small droplets. In many cases, the droplets were covered with dust and did not exhibit the characteristic silver color. Other droplets were difficult to detect because the floor grating was either galvanized or painted silver.

The data and source of the spill have not been determined but the spill is likely to be the result of human error. The failure to report the spill violated the requirements of the spill response procedure contained in the building emergency plan, resulting in unnecessary personnel exposures.

There was no measurable release to the environment. The room is a surface contamination area, and the potential for hazardous waste spills was previously recognized. All liquid waste spilled to the floor drains 
to a double-shell underground storage tank (241-AW-102). Personnel who were potentially exposed to the mercury are currently being evaluated to determine if mercury was absorbed in hazardous amount.

The spill will impact on the 242-A evaporator start-up schedule. The training run, which was in progress when the spill was discovered, has been interrupted and will be delayed until the clean-up is completed.

Corrective actions include completion and issuance of safety plans and emergency response plans pertaining to spills, as well as additional training drills and the review of spill response equipment.

Changes to the Report:

1. Summary section

The Integrated Core Sample Schedule, formerly Figure 1, will no longer appear in this report until the new schedule has been revised and approved.

Figure 2, Master Core Sampling Schedule, is included only in this issue to show individual tank core sampling and scheduling information for $F Y$ 1992 through 1998.

2. Section A, Tank Equipment Code and Status Definitions

A subsection category, Waste Tanks - General, has been added to item number 3, Definitions. 

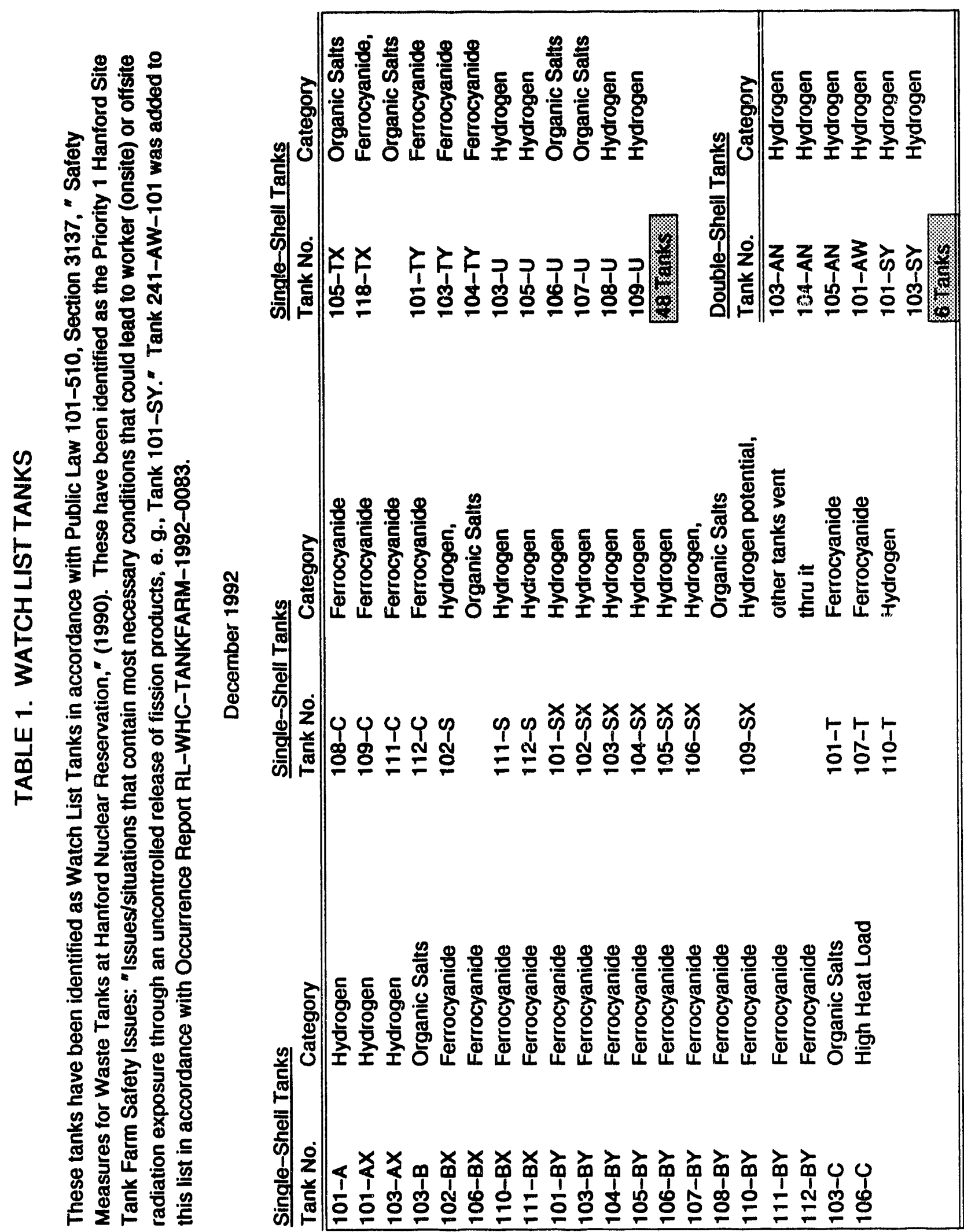


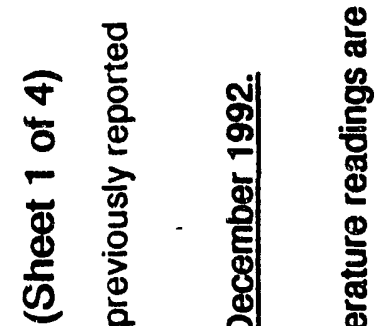

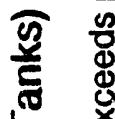

흐

릉

3

$\sum$

늘

$<\frac{1}{9}$

$(6)$

8

正

4 ठิ

岁?

핑응

$\Sigma$

2

$\frac{5}{10}$

0 ஸ

용 홍

人 离

ऽ

这

( )

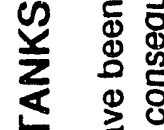

$\leftarrow \sum_{0}^{0}$

ก

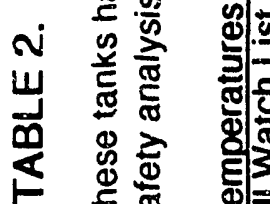

WHC-EP-0182-57

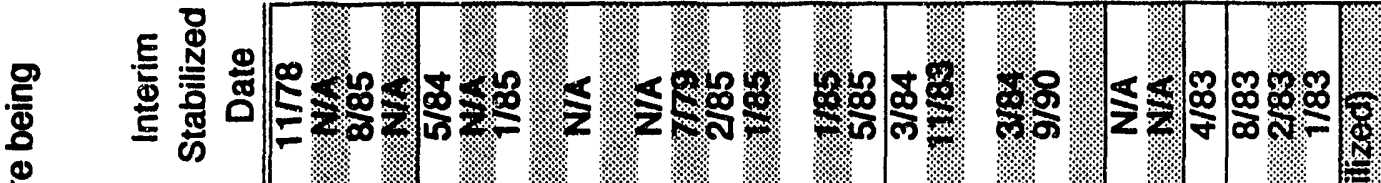

E

을

का

들 원

क्षे

융

응

วิ

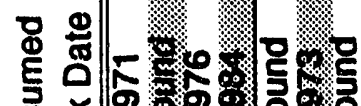

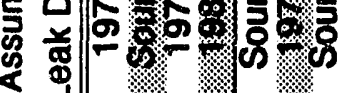

\$:

8.

:

พ

ö의.

⿹勹巳

of

한

莺

8. $8.80 \%$

\%

$\frac{58}{80}$

$8.08 .8 \%$

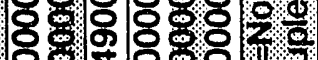

${ }^{2}$

ลิํํำ

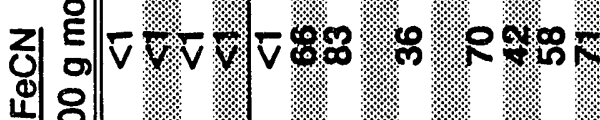

Cov

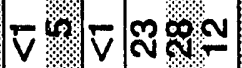

敢

.

สั

कุ

흉 논

$\frac{0}{x}$

$\sum$

트름

क्ष

क

ㅎํㅇ

0

오음

든 응

을.

올

동

म

造

웋

\&

등 들

을

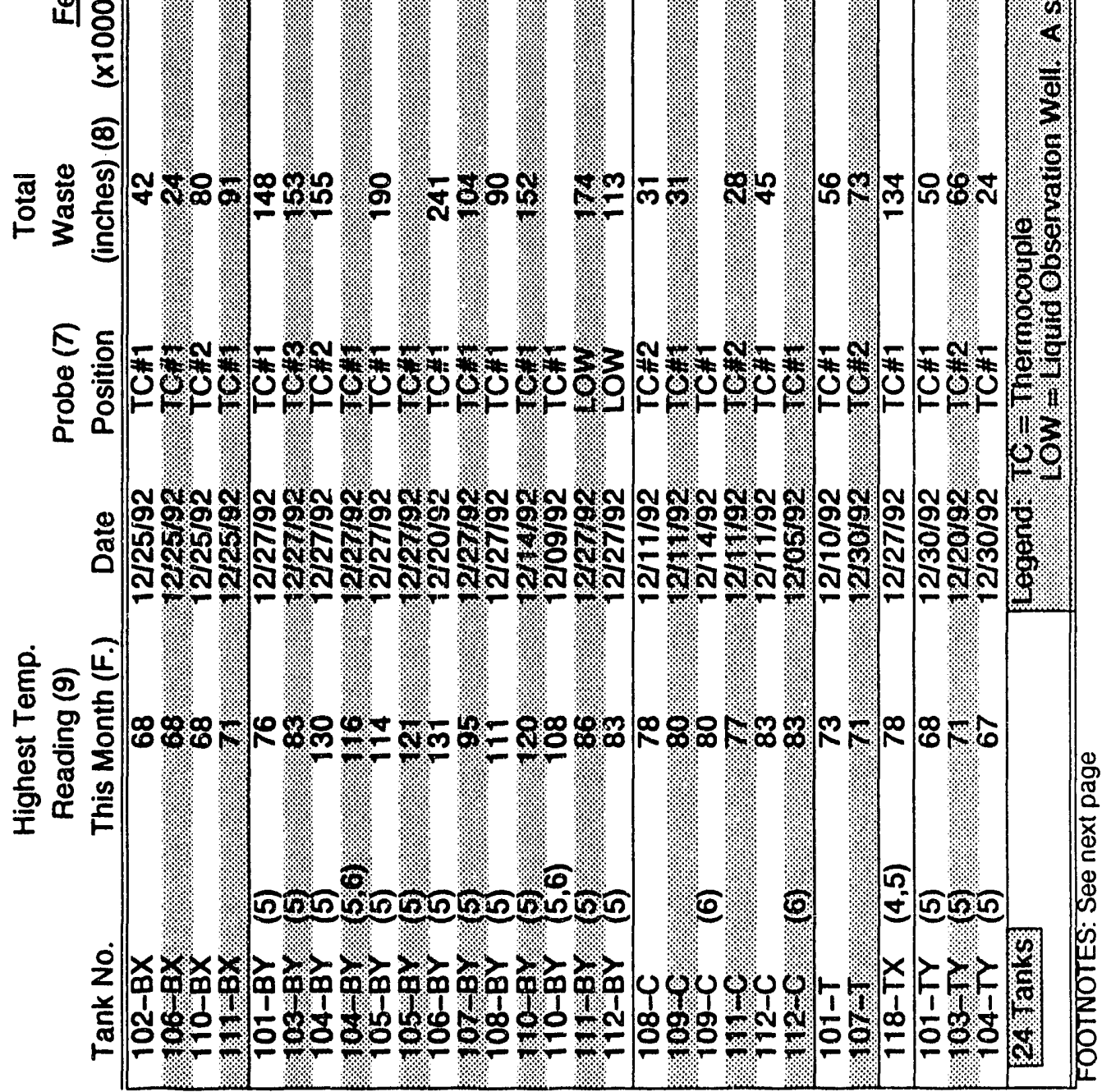




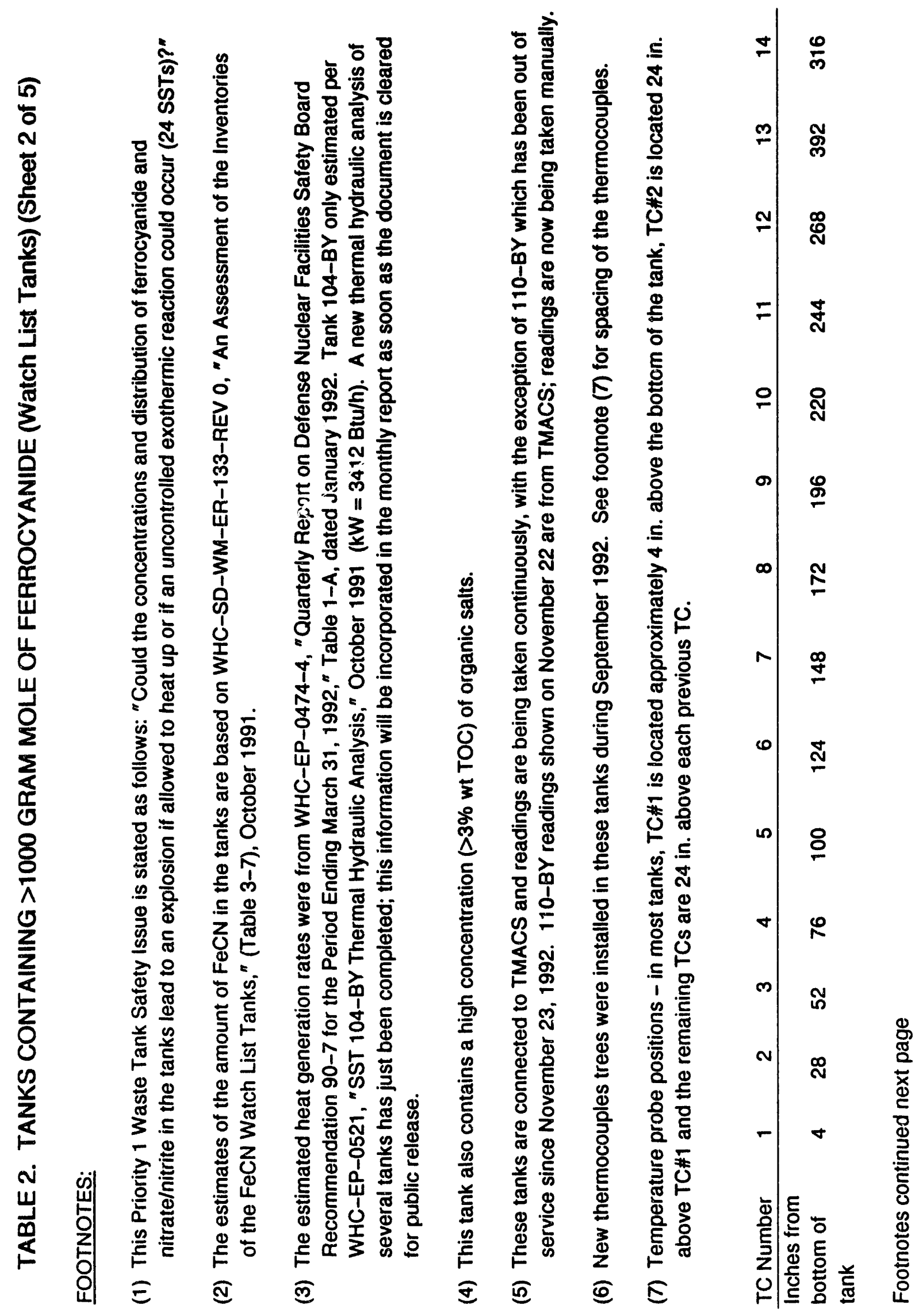



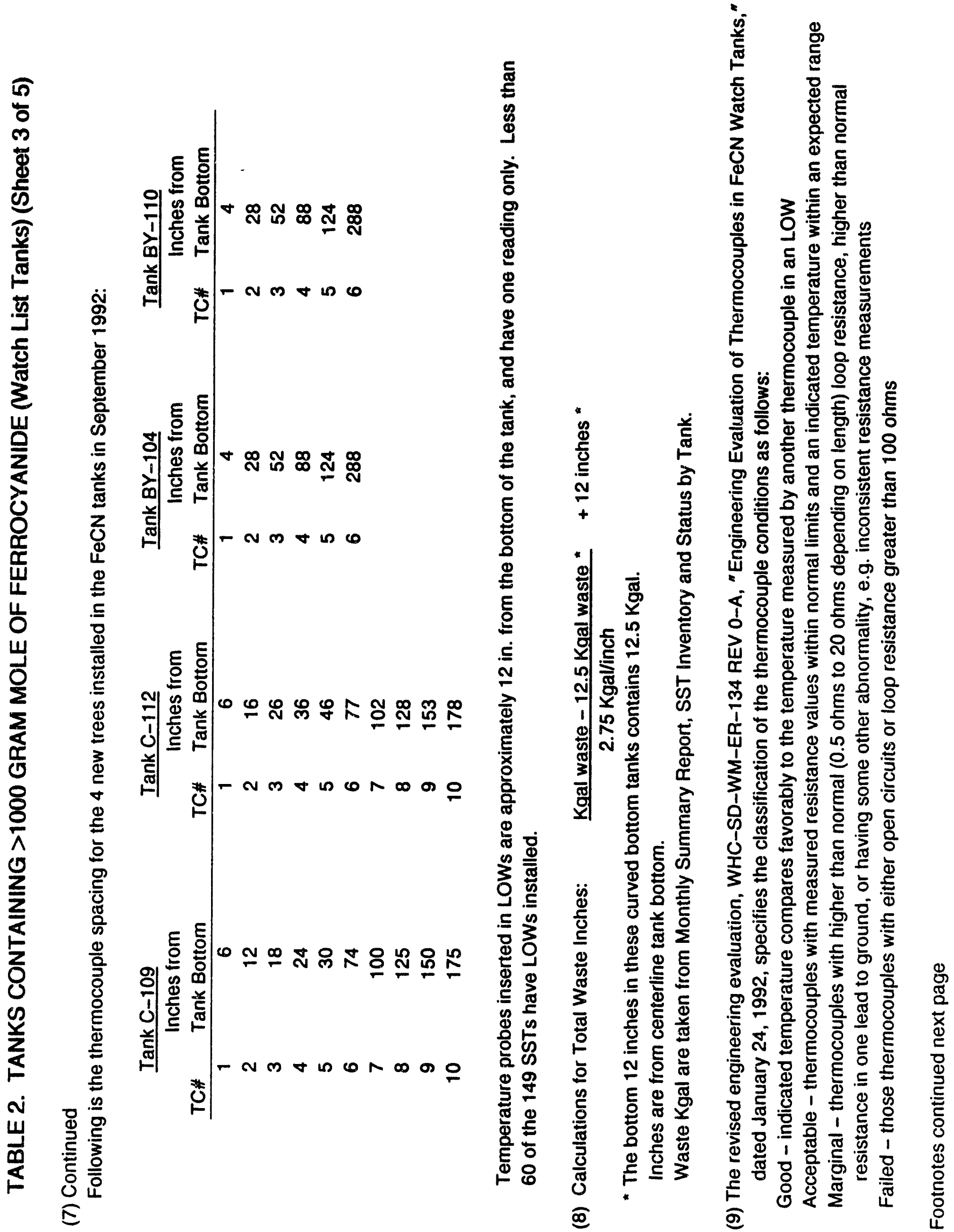


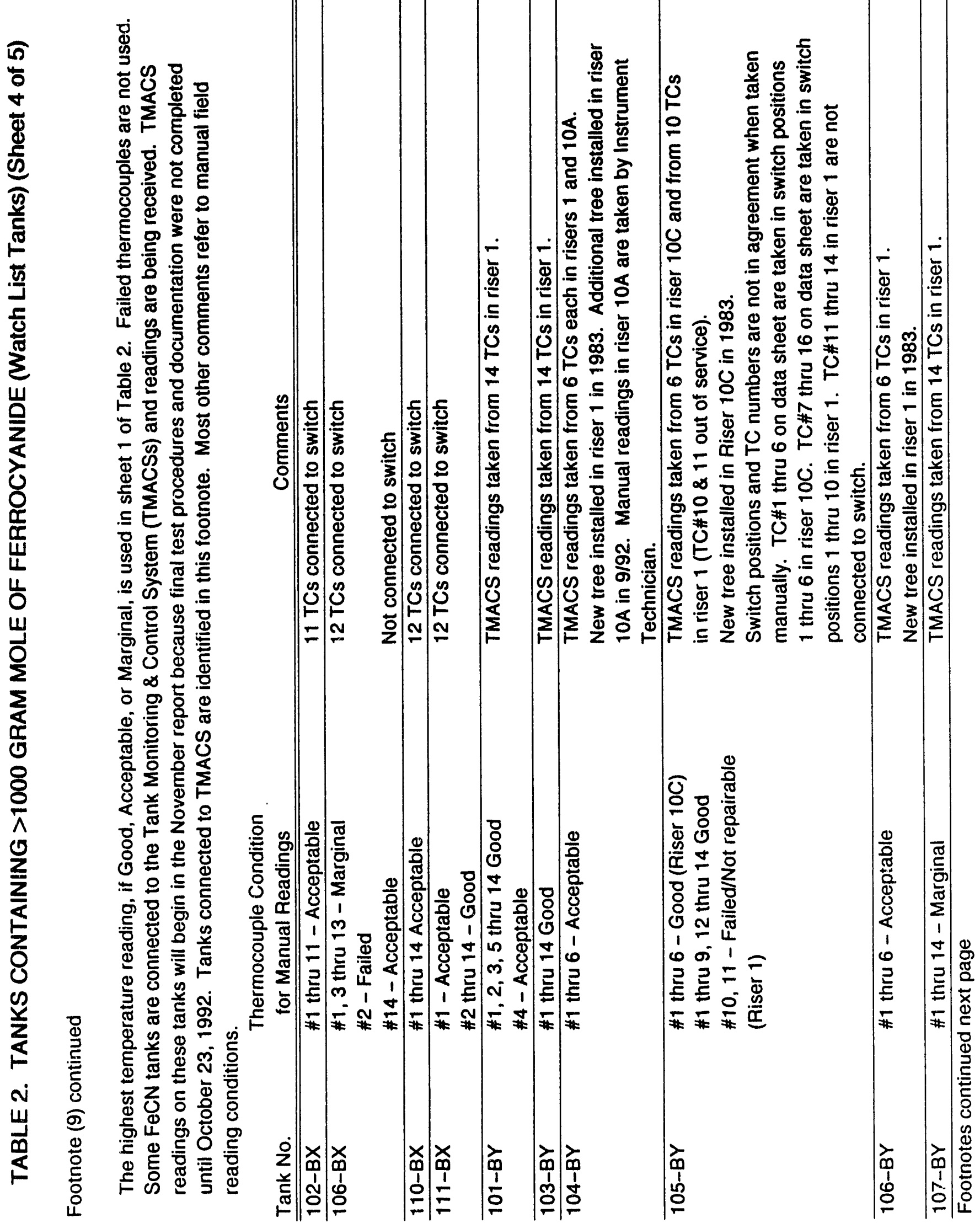




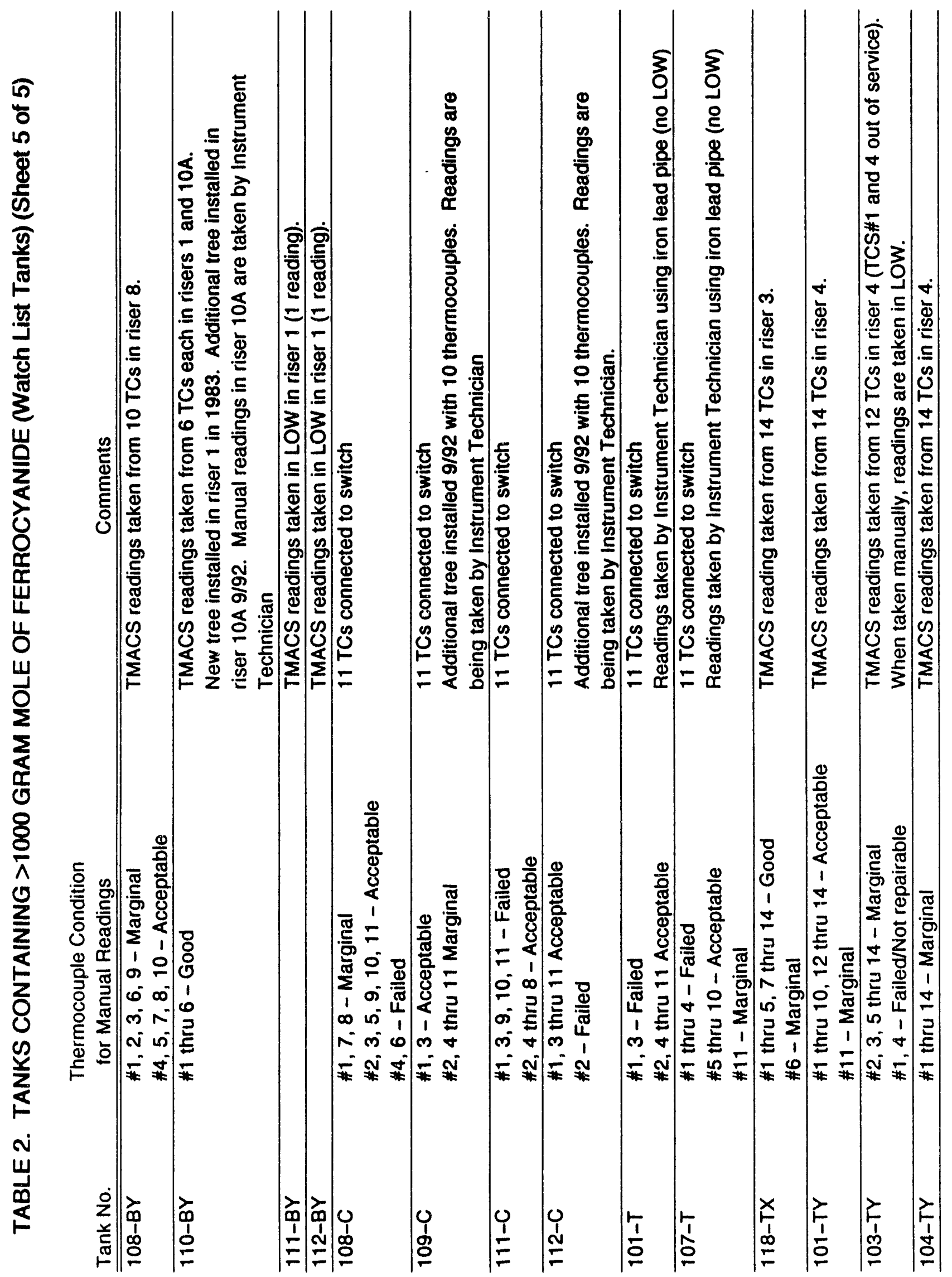



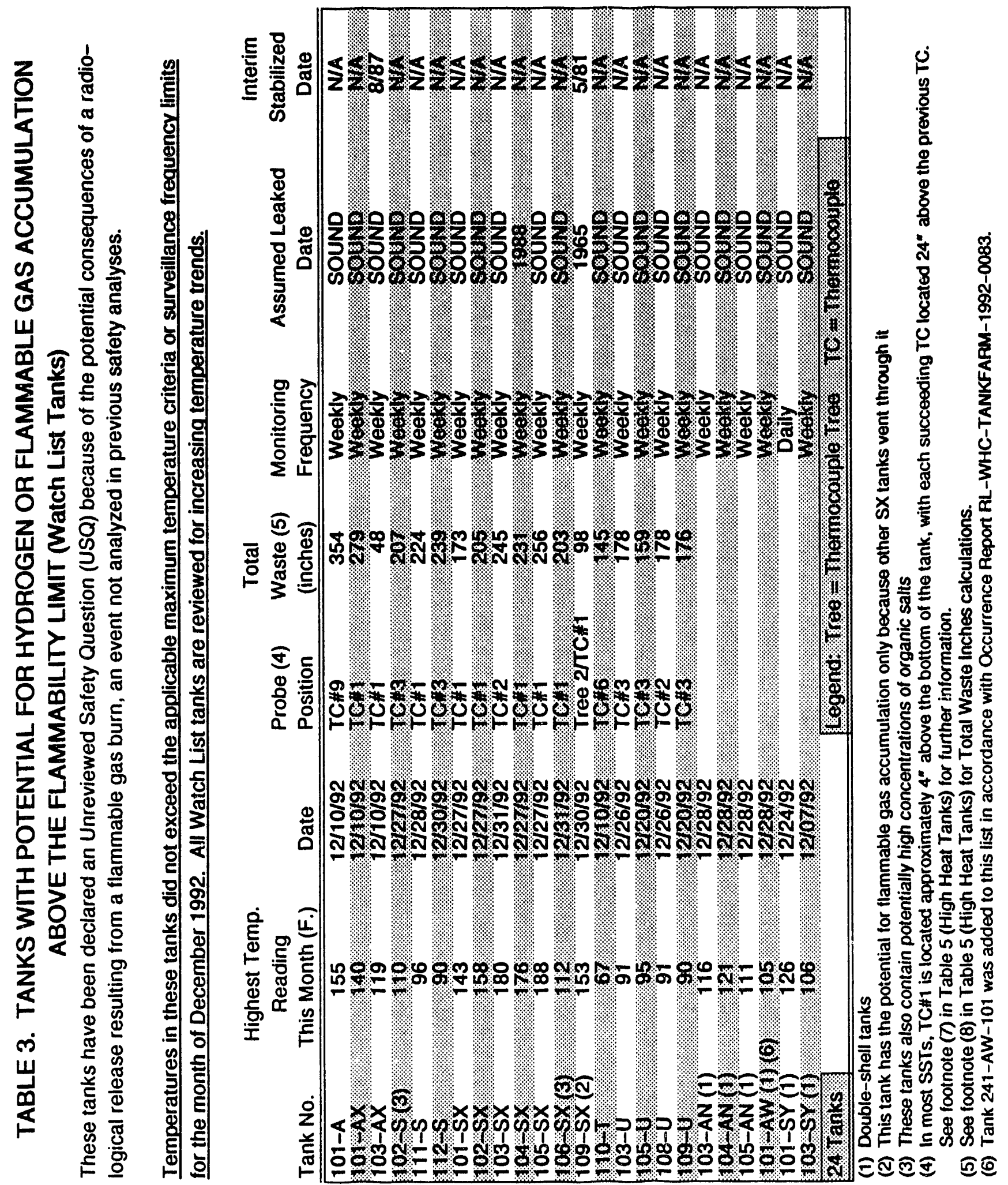

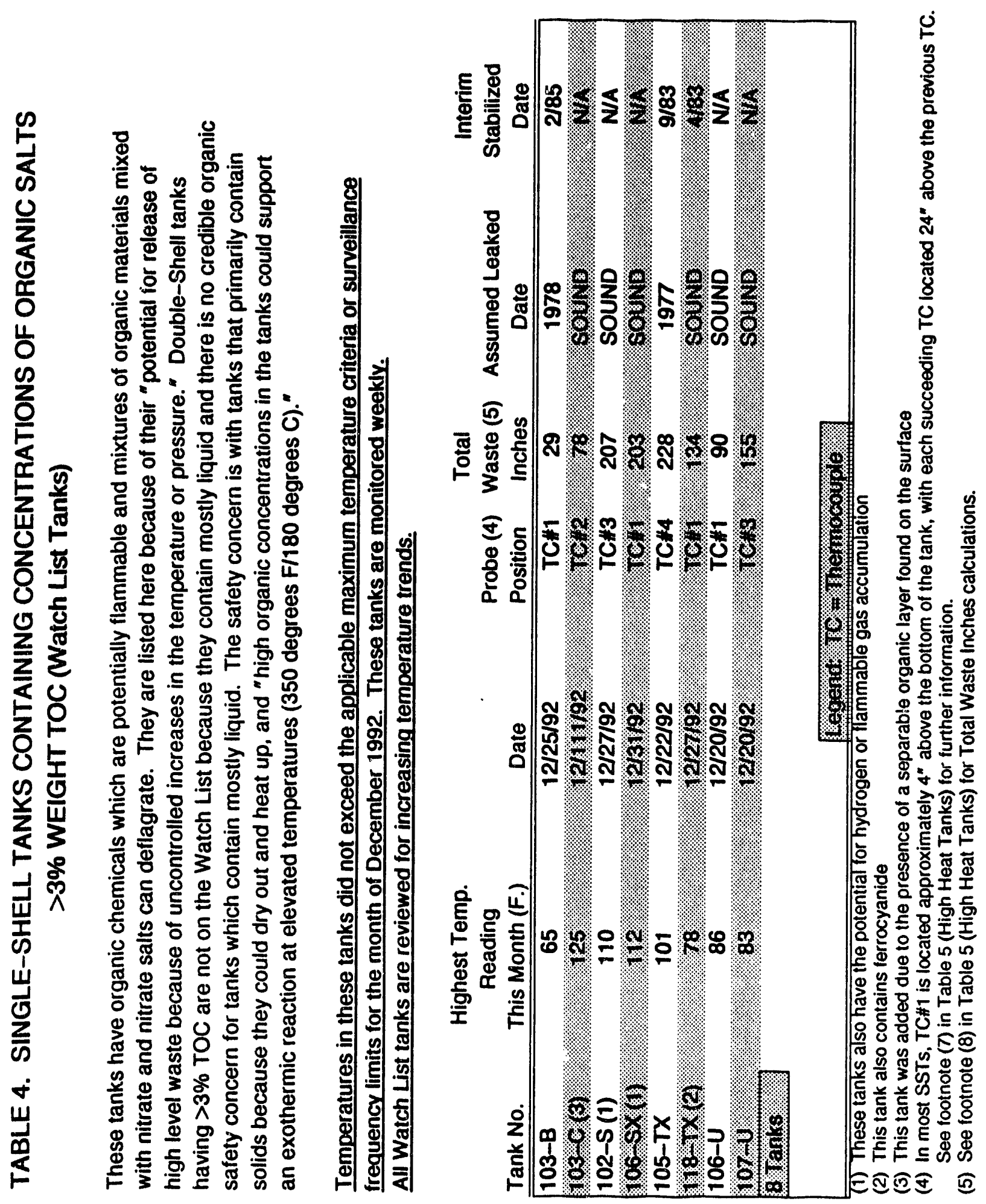


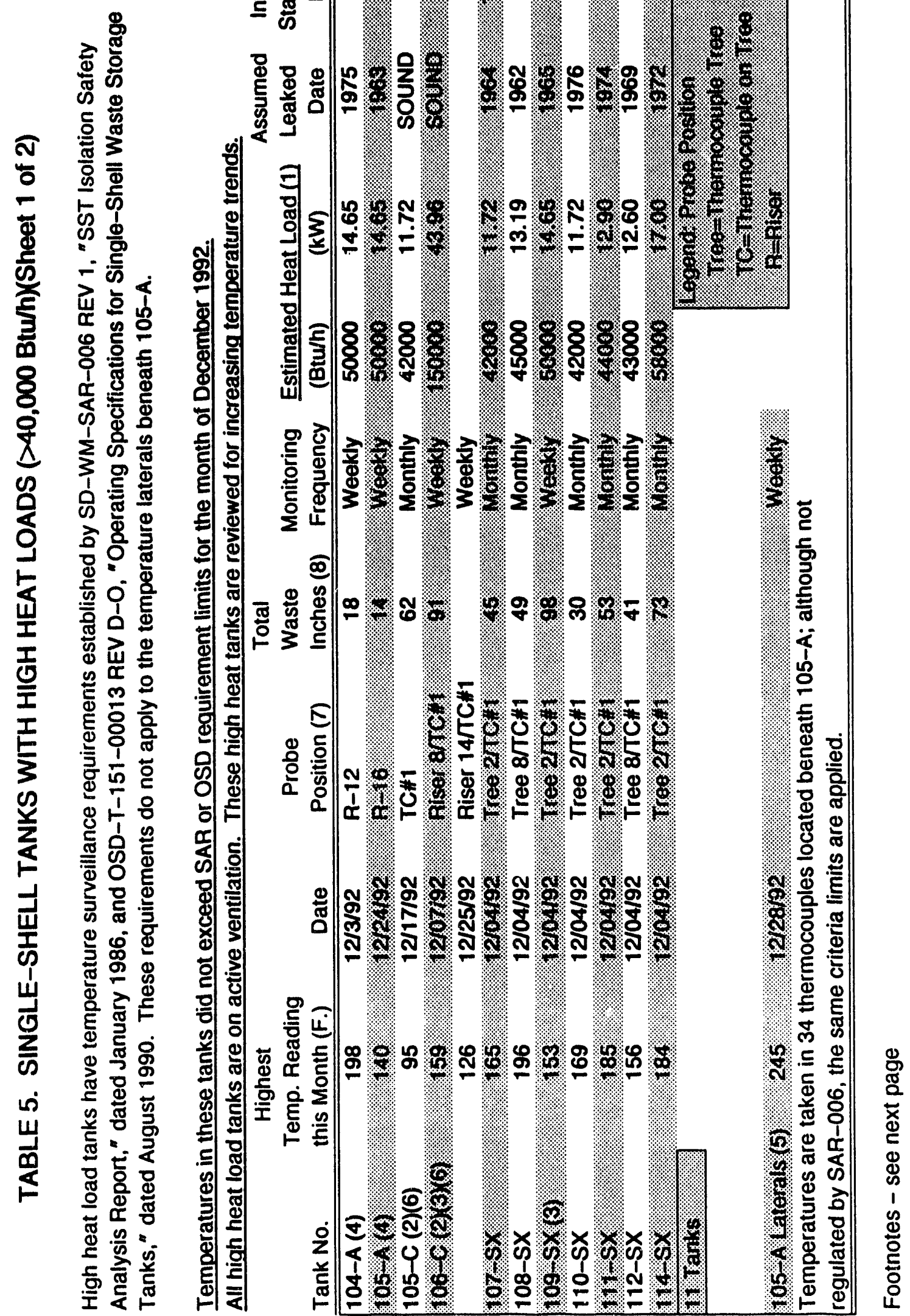




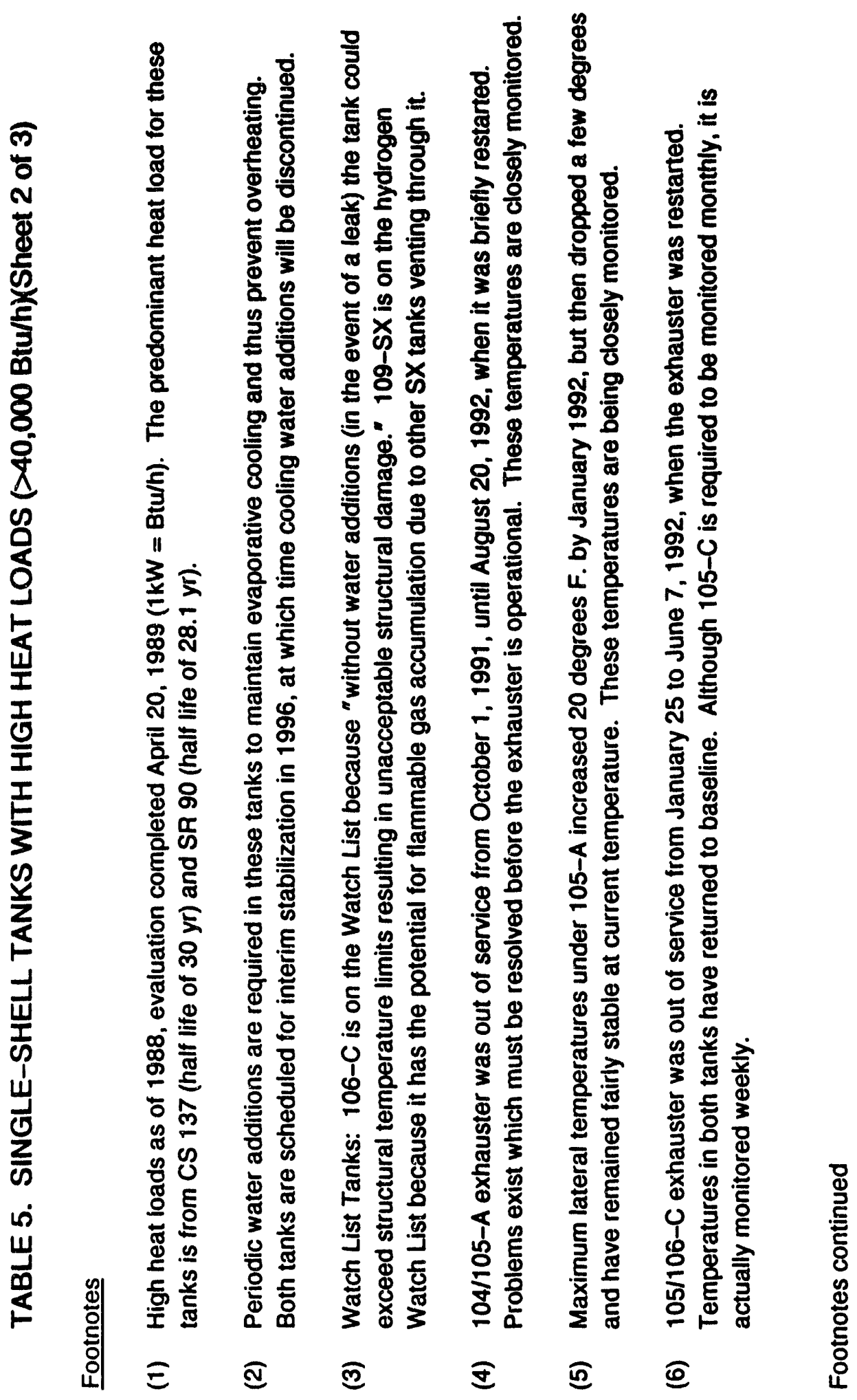




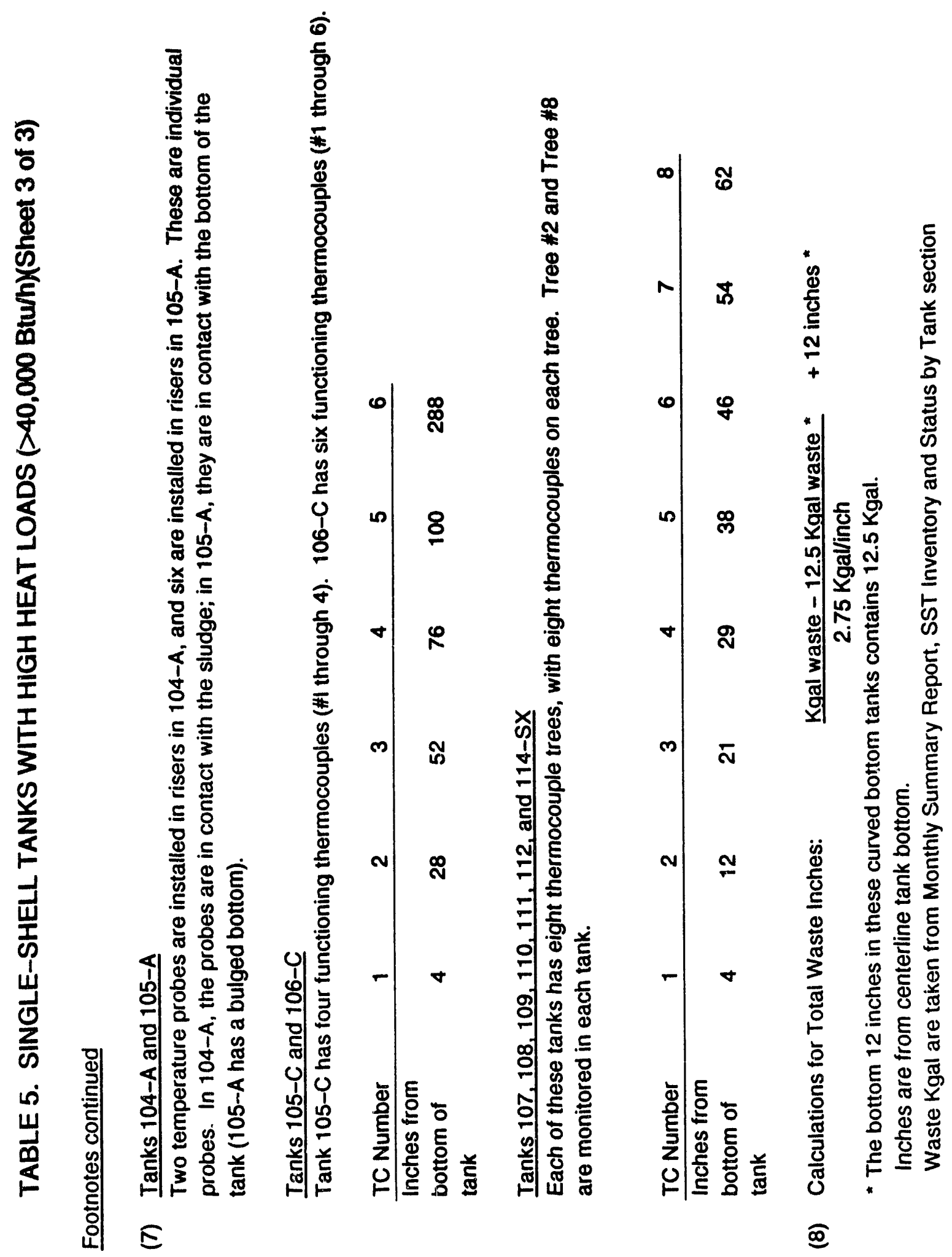




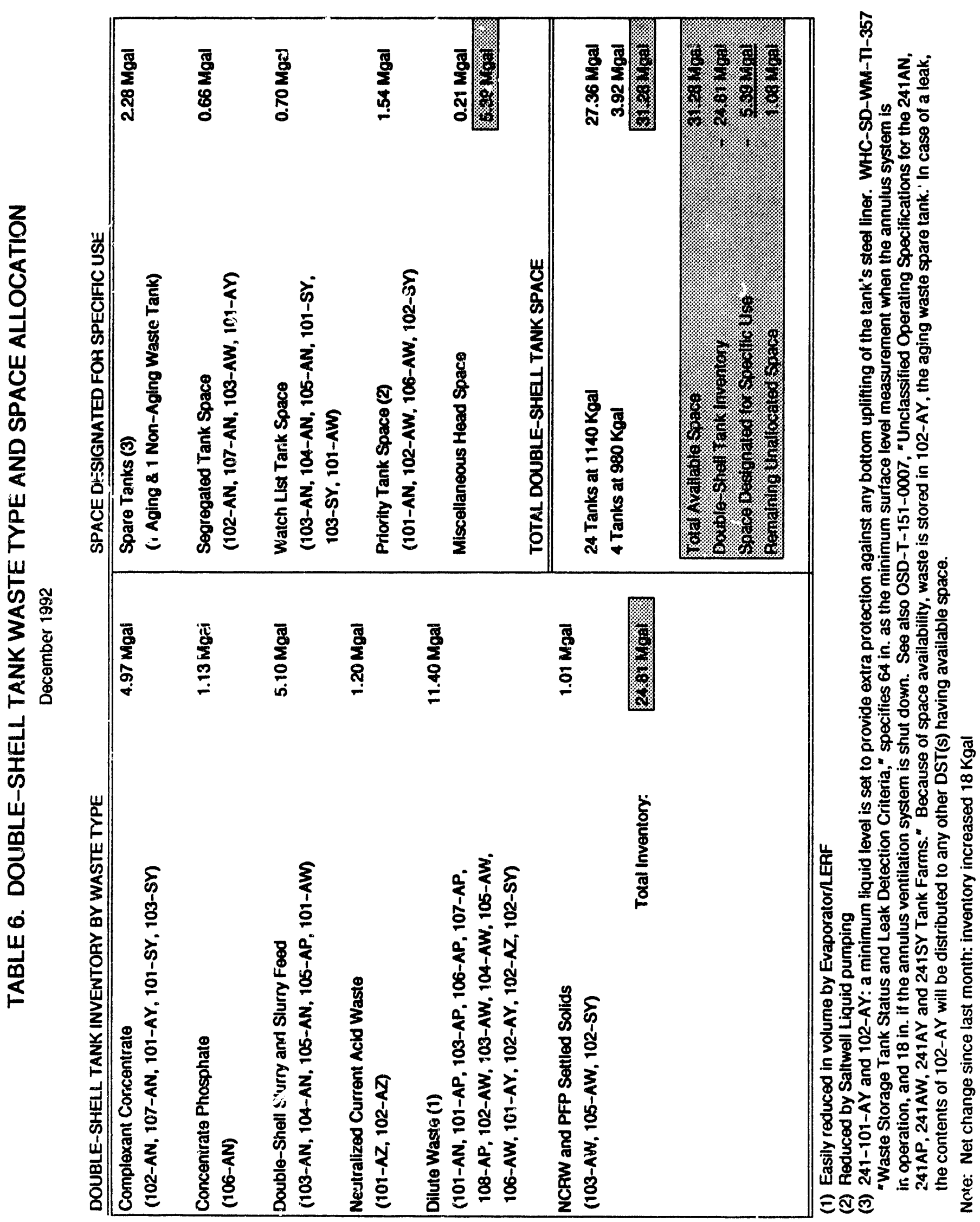




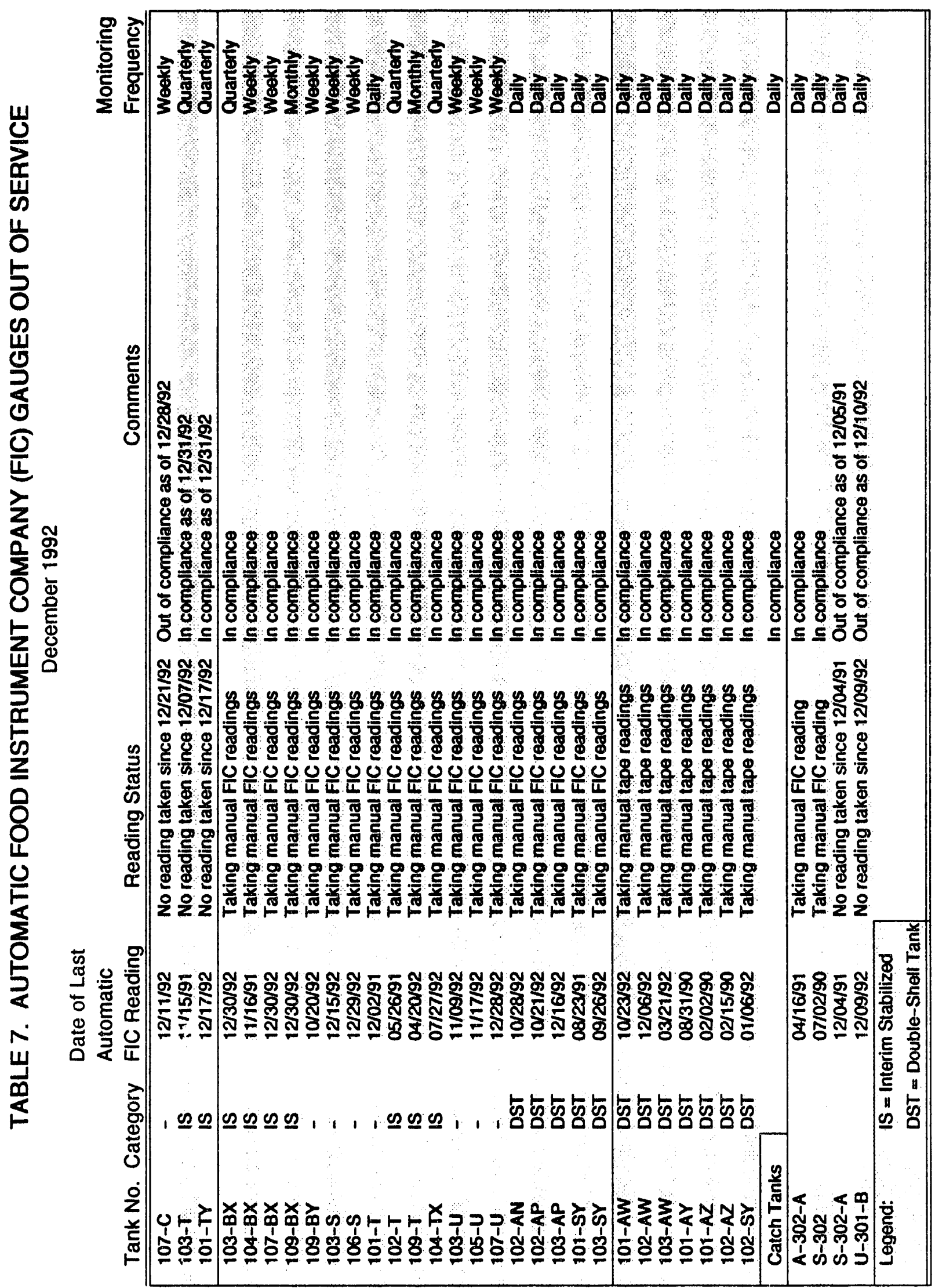




\section{TABLE 8. SINGLE-SHELL TANKS MONITORING COMPLIANCE STATUS 149 TANKS (Sheet 1 of 4)}

The following table indicates whether Single-Shell tank monitoring is in compliance with the requirements as specified in the applicable documents:

NOTE:

All Watch List and High Heat tank temperature monitoring is in compliance.

All Dome Elevation Survey monitoring is in compliance.

All Drywell monitoring is in compliance.

Psychrometrics (2)

In-tank Photographs (3)

\begin{tabular}{|c|c|}
\hline \multicolumn{2}{|l|}{ LEGEND: } \\
\hline 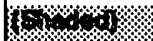 & = In compliance with all applicable documentation \\
\hline OE & $=$ Out of compliance with documentation \\
\hline-357 & $\begin{array}{l}=\text { WHC-SD-WM-TI-357, "Waste Storage Tank Status and Leak } \\
\text { Detection Criteria" }\end{array}$ \\
\hline POP & $\begin{array}{l}\text { - Plant Operating Procedure TO-040-650, "Obtain/Record SST } \\
\text { Temperatures" }\end{array}$ \\
\hline M.T. & = Manual Tape \\
\hline FIC & = Food Instrument Company \\
\hline OSRISAR & $\begin{array}{l}\text { - Operations Safety Requirements/Safety Analysis Report } \\
\text { Requirements }\end{array}$ \\
\hline OSD & - Operating Specifications Document \\
\hline N/A & $=$ Not Applicable (i.e., no LOW, M.T, FIC inctalled) \\
\hline o/s & - Out of Service \\
\hline Gamma & = LOW readings taken by Gamma probe \\
\hline Neutron & - LOW readings taken by Neutron probe \\
\hline
\end{tabular}

Information as of $12 / 31 / 92$

\begin{tabular}{|c|c|c|c|c|c|c|c|c|c|c|}
\hline \multirow{4}{*}{$\begin{array}{l}\text { Tank } \\
\text { Number }\end{array}$} & \multirow{2}{*}{\multicolumn{2}{|c|}{ Category }} & \multirow{4}{*}{$\begin{array}{l}\text { Temperature } \\
\text { Readings (5) } \\
\end{array}$} & \multirow{3}{*}{\multicolumn{2}{|c|}{$\begin{array}{c}\text { Surface Level } \\
\text { Readings (1) } \\
(-357)\end{array}$}} & \multirow{3}{*}{\multicolumn{2}{|c|}{$\begin{array}{c}\text { LOW Readings } \\
(-357)\end{array}$}} & \multicolumn{2}{|c|}{ Radiation Readings } & \multirow{4}{*}{$\begin{array}{l}\text { Dome } \\
\text { Elevation } \\
\text { Surveys } \\
\text { (OSR SAR) } \\
\end{array}$} \\
\hline & & & & & & & & \multirow{3}{*}{$\begin{array}{c}\text { Lateral } \\
\text { Readings } \\
(-357) \\
\end{array}$} & \multirow{3}{*}{$\begin{array}{c}\text { Drywell } \\
\text { Readings } \\
\text { (OSR/SAR) } \\
\end{array}$} & \\
\hline & \multirow{2}{*}{$\begin{array}{c}\text { Watch } \\
\text { List }\end{array}$} & \multirow{2}{*}{$\begin{array}{l}\text { High } \\
\text { Heat }\end{array}$} & & & & & & & & \\
\hline & & & & M.T. & FC & Gamma & Neutron & & & \\
\hline $101-A$ & $x$ & & & & 11 & & & O/C & & \\
\hline $102-A$ & & & & $11 \%$ & & 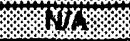 & 10 & O/C & & \\
\hline $103-A$ & & & & Kro & & & & O/C & & \\
\hline $104-A$ & & $\bar{x}$ & & & $1 \%$ & $2 \%$ & 10 & O/C & & \\
\hline $105-A$ & & $\bar{x}$ & & & \%ra & 21. & 10 & $\mathrm{O} / \mathrm{C}$ & & \\
\hline $106-A$ & & & & 140 & & 10 & 30 & O/C & & \\
\hline $101-A X$ & $\bar{x}$ & & & $871 \%$ & & & & & & \\
\hline 102-AX & & & & & w1 & $4=$ & N- & & & \\
\hline $103-A X$ & $\bar{x}$ & & & $80 \%$ & & $10 \%$ & ore & & & \\
\hline 104-AX & & & & & 14 & $x+1$ & 10 & & & \\
\hline $101-B$ & & & & morn & & 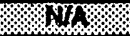 & 19 & & & \\
\hline $102-B$ & & & & 4 & & 4 & $4 \%$ & & & \\
\hline $103-B$ & $\bar{x}$ & & & $2 \%$ & & $\mathrm{Nu}$ & 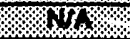 & & & \\
\hline 104-B & & & & & 44 & & 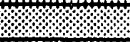 & & & \\
\hline $105-B$ & & & & & Nos. & & & & & \\
\hline $106-B$ & & & & Ut & & 141 & Ny & & & \\
\hline $107-B$ & & & & & WII: & N1\% & Nirs & & & \\
\hline $108-8$ & & & & $14 \%$ & & 10 & No: & & & \\
\hline $109-B$ & & & & & 100 & XNA & NNA & & & \\
\hline $110-B$ & & & & & 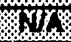 & $\mathrm{ND}$ & N/ & & & \\
\hline $111-B$ & & & & sin & & No & NUK & & & \\
\hline $112-B$ & & & & 111 & & 10 & 101 & & & \\
\hline $201-B$ & & & & & 61 & $\mathrm{No}$ & $\mathrm{NR}$ & & & \\
\hline $202-3$ & & & & & 40 & N11 & $14 \mathrm{~s}$ & & & \\
\hline $203-B$ & & & & & 102 & 80 & $\mathrm{NA}$ & & & \\
\hline $204-B$ & & & & & 47 & W1: & 10 & & & \\
\hline $\mid 101-B X$ & & & & & 611 & 111\% & $\mathrm{SWM}$ & & & \\
\hline $\mid$ & $\bar{x}$ & & & & wor & 118 & 14. & & & \\
\hline $103-B X$ & & & OIC & $N /$ & & Wu & ofr & & & \\
\hline 104-BX & & & O/S-O/C & $\mathrm{NH}$ & & 40 & 10 & & $\%$ & \\
\hline 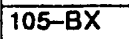 & & & & NK & & WNA & $\mathrm{NTA}$ & & & \\
\hline $106-B X$ & $\bar{x}$ & & & No & & NA & NHA & & $1 \%$ & \\
\hline $107-\mathrm{BX}$ & & & O/C & NII & & $\mathrm{Nu}$ & N/A & & & \\
\hline
\end{tabular}


TABLE 8. SINGLE-SHELL TANKS MONITORING COMPLIANCE STATUS 149 TANKS (Sheet 2 of 4 )

Information as of $12 / 31 / 92$

\begin{tabular}{|c|c|c|c|c|c|c|c|c|c|c|}
\hline \multirow{4}{*}{$\begin{array}{l}\text { Tank } \\
\text { Number }\end{array}$} & \multirow{2}{*}{\multicolumn{2}{|c|}{ Category }} & \multirow{4}{*}{$\begin{array}{l}\text { Temperature } \\
\text { Readings (5) }\end{array}$} & \multirow{3}{*}{\multicolumn{2}{|c|}{$\begin{array}{c}\text { Surface Level } \\
\text { Readings (1) } \\
\text { (-357) }\end{array}$}} & \multirow{3}{*}{\multicolumn{2}{|c|}{$\begin{array}{c}\text { LOW Readings } \\
(-357)\end{array}$}} & \multicolumn{2}{|c|}{ Radiation Readings } & \multirow{4}{*}{$\begin{array}{c}\text { Dome } \\
\text { Elevation } \\
\text { Surveys } \\
\text { (OSR/SAR) }\end{array}$} \\
\hline & & & & & & & & \multirow{3}{*}{\begin{tabular}{|c|} 
Lateral \\
Readings \\
$(-357)$
\end{tabular}} & \multirow{3}{*}{$\begin{array}{c}\text { Drywell } \\
\text { Readings } \\
\text { (OSR/SAR) }\end{array}$} & \\
\hline & \multirow{2}{*}{$\begin{array}{c}\text { Watch } \\
\text { List }\end{array}$} & \multirow{2}{*}{$\begin{array}{l}\text { High } \\
\text { Heat }\end{array}$} & & & & & & & & \\
\hline & & & & M.T. & FIC & Gamma & Neutron & & & \\
\hline $108-\mathrm{BX}$ & & & $\overline{\mathrm{OK}}$ & & $2 \%$ & 8 & $8 x$ & $4 \%$ & & \\
\hline 108-BX & & & OK & $8 \%$ & & $81 \%$ & $8 \%$ & 1018 & & \\
\hline $110-B X$ & $\bar{x}$ & & & & 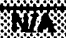 & 43 & 42 & 40 & & \\
\hline $111-\mathrm{BX}$ & $\bar{x}$ & & & & 1018 & & & 414 & & \\
\hline $112-B X$ & & & OKC & $y$ & & $8 \%$ & 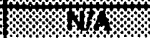 & 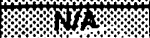 & & \\
\hline 101-BY & $\bar{x}$ & & & & 168 & & & 648 & & \\
\hline $102-B Y$ & & & O/S-O/C & & $4 \%$ & & & 14 & & \\
\hline 103-BY & $\bar{x}$ & & & & $x$ & & & $82 \%$ & & \\
\hline 104-BY & $\bar{x}$ & & & & 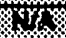 & & & $4 \%$ & & \\
\hline 105-BY & $\bar{x}$ & & & & 16 & & & $1 \%$ & & \\
\hline 108-BY & $\bar{X}$ & & & & $\%$ & & & 4 & & \\
\hline $107-B Y$ & $\bar{x}$ & & & & $10 \%$ & & & NH & & \\
\hline $108-B Y$ & $\bar{x}$ & & & & 8 & $8 \%$ & $8 \%$ & 4 & & \\
\hline 109-BY & & & $\mathrm{O} / \mathrm{S}-\mathrm{O} / \mathrm{C}$ & $8 M$ & & & & NO & & \\
\hline $110-B Y$ & $\bar{x}$ & & & & 10 & & & 14 & & \\
\hline $111-B Y$ & $\bar{x}$ & & & & 80 & & & $\mathrm{~N} / \mathrm{A}$ & & \\
\hline 112-BY & $\bar{x}$ & & & & 4 & O/C & & N4 & & \\
\hline $101-C$ & & & & & 10 & 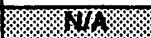 & 114 & $N \mathrm{~N}$ & & \\
\hline $102-C$ & & & & 4 & & 4 & 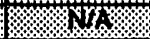 & W1 & $N A$ & \\
\hline $103-C$ & $\bar{x}$ & & & $14 \pi$ & & 10 & 10 & $\mathrm{NH}$ & & \\
\hline $104-C$ & & & & 84 & & 41 & 81 & $\mathrm{N1}$ & & \\
\hline $105-C$ & & $\bar{x}$ & & $8 \mathrm{ST}^{\circ}$ & & $1 / 1$ & $\mathrm{NIA}$ & $\mathrm{NtA}$ & & \\
\hline $106-C(4)$ & $\bar{x}$ & $\bar{x}$ & & 14 & & $4 \%$ & 28 & 34 & & \\
\hline $107-C$ & & & & 101 & O/C & 142 & 818 & $\mathrm{~N} / \mathrm{A}$ & & \\
\hline $108-C$ & $\bar{x}$ & & & & $8 \times$ & 84 & $42 \%$ & NH & & \\
\hline $100-\mathrm{C}$ & $\bar{x}$ & & & & 10 & $11 / 2$ & $8 \mathrm{NIA}$ & $8 \mathrm{NA}$ & & \\
\hline $110-C$ & & & & & 0 & $\mathrm{NHA}$ & $N 4$ & $N A$ & & \\
\hline $111-C$ & $\bar{x}$ & & & & $\mathrm{~N} \mathrm{NA}$ & 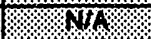 & 10 & $\mathrm{NA}$ & & \\
\hline $112-C$ & $\bar{x}$ & & & & 14 & $11 \%$ & 40 & $\mathrm{NH}$ & & \\
\hline $201-C$ & & & & & 14 & $\mathrm{ma}$ & 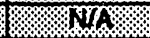 & $\mathrm{NH}$ & & \\
\hline $202-C$ & & & & & 4 & 418 & 14 & 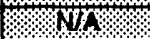 & & \\
\hline $203-C$ & & & & & sur & 621 & 1018 & $N \mathrm{NA}$ & & \\
\hline $204-C$ & & & $\mathrm{O} / \mathrm{C}$ & & $4 \%$ & 47 & 84 & $N A$ & & \\
\hline $101-5$ & & & & $1 \%$ & & & & 80 & & \\
\hline $102-5$ & $\bar{x}$ & & & 41 & & & & 40 & & \\
\hline $103-5$ & & & & $\mathrm{NT}$ & & & & $\mathrm{NI}$ & & \\
\hline $104-S$ & & & & & $4 \%$ & 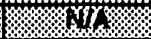 & 40 & 14 & & \\
\hline $105-S$ & & & & $87 \%$ & & & & NTH & & \\
\hline $108-5$ & & & & 14 & & & & 104 & & \\
\hline $107-5$ & & & & 11 & & 10 & 14 & $N I A$ & & \\
\hline $108-5$ & & & & & & & & NHA & & \\
\hline $109-5$ & & & & $N I A$ & & & & NTA & & \\
\hline $110-5$ & & & & 4 & & & & NA & & \\
\hline $111-S$ & $x$ & & & $14 \%$ & & & & $\mathrm{NA}$ & & \\
\hline 112-S & $x$ & & & $N T$ & & & & NA & & \\
\hline $101-S X$ & $\bar{x}$ & & & $\mathrm{NH}$ & & & & NA & & \\
\hline $102-5 X$ & $x$ & & & $4 t$ & & & & $N A$ & & \\
\hline $103-5 x$ & $\bar{x}$ & & & 41 & & & & $\mathrm{~N} / \mathrm{A}$ & & \\
\hline 104-SX & $\bar{x}$ & & & 41 & & O/S-O/C & O/S-O/C & $\mathrm{NHA}$ & & \\
\hline $105-5 x$ & $\bar{x}$ & & & $N M$ & & & & O/C & & \\
\hline $106-5 X$ & $\bar{x}$ & & & 40 & & & & $\mathrm{NAP}$ & & \\
\hline $107-5 X$ & & $\bar{x}$ & & & WNI & NI & $\mathrm{Nt}$ & $\mathrm{O} / \mathrm{C}$ & & \\
\hline $108-5 x$ & & $\bar{x}$ & & & 44 & 10 & 10 & $\mathrm{OIC}$ & & \\
\hline
\end{tabular}


TABLE 8. SING: E-SHELL TANKS MONITORING COMPLIANCE STATUS 149 TANKS (Sheet 3 of 4 )

Information as of $12 / 31 / 92$

\begin{tabular}{|c|c|c|c|c|c|c|c|c|c|c|}
\hline \multirow{4}{*}{$\begin{array}{l}\text { Tank } \\
\text { Number }\end{array}$} & \multirow{2}{*}{\multicolumn{2}{|c|}{ Category }} & \multirow{4}{*}{$\begin{array}{l}\text { Temperature } \\
\text { Readings (5) }\end{array}$} & \multirow{3}{*}{\multicolumn{2}{|c|}{$\begin{array}{c}\text { Surface Level } \\
\text { Peadings (1) } \\
\text { (-357) }\end{array}$}} & \multirow{3}{*}{\multicolumn{2}{|c|}{$\begin{array}{c}\text { LOW Readings } \\
(-357)\end{array}$}} & \multicolumn{2}{|c|}{ Radiation Readings } & \multirow{4}{*}{$\begin{array}{l}\text { Dome } \\
\text { Elevation } \\
\text { Surveys } \\
\text { (OSR/SAR) }\end{array}$} \\
\hline & & & & & & & & \multirow{3}{*}{$\begin{array}{c}\text { Lateral } \\
\text { Readings } \\
(-357)\end{array}$} & \multirow{3}{*}{$\begin{array}{c}\text { Drywell } \\
\text { Readings } \\
\text { (OSR/SAR) }\end{array}$} & \\
\hline & \multirow{2}{*}{\begin{tabular}{|c|} 
Watch \\
List \\
\end{tabular}} & \multirow{2}{*}{$\begin{array}{l}\text { High } \\
\text { Heat }\end{array}$} & & & & & & & & \\
\hline & & & & M.T. & FTC & Gamma & Neutron & & & \\
\hline $100-5 \times(4)$ & $\overline{\bar{x}}$ & $\bar{x}$ & & & WYY & $8 y \%$ & $\overline{X Y X X}$ & $\overline{O / C}$ & & \\
\hline $110-S x$ & & $\bar{x}$ & & & 2 & $m$ & $8 \%$ & O/C & & \\
\hline $111-s x$ & & $\bar{x}$ & & & 14 & $1 \%$ & $\%$ & O/C & & \\
\hline $112-S X$ & & $\bar{x}$ & & & If & 8 & $8 \%$ & O/C & & \\
\hline $113-S X$ & & & & & X & 16 & $14 \%$ & 143 & & \\
\hline 114-SX & & $\bar{x}$ & & & m & $1 \mathrm{Mr}$ & $801 \%$ & O/C & & \\
\hline $115-S X$ & & & O/S - OKC & & 14 & 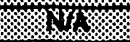 & 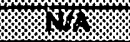 & O/C & & \\
\hline $101-\mathrm{T}$ & $\bar{x}$ & & & $3 \%$ & & $m$ & m & $10 \%$ & & \\
\hline $102-T$ & & & OIS-O/C & 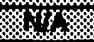 & & $1 \%$ & 14 & 4 & & \\
\hline 103-T & & & & $107 \%$ & & $8 \%$ & \% & NTS & & \\
\hline 104-T & & & & & 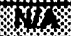 & & & 40 & & \\
\hline $105-T$ & & & $0 / \mathrm{S}-0 / \mathrm{C}$ & 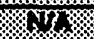 & & 192 & $8 \pi$ & 10 & & \\
\hline $108-T$ & & & & 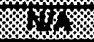 & & 16 & 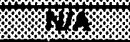 & 4 & & \\
\hline $107-T$ & $\bar{x}$ & & & IIIIX & & ora & $8 \pi$ & NTS & & \\
\hline $108-T$ & & & OIC & & 10 & $4 \times$ & $4+2$ & $4 \%$ & & \\
\hline $108-T$ & & & O/C & 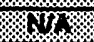 & & 101 & NT & WrA & & \\
\hline $110-T$ & $\bar{x}$ & & & $x$ & $\%$ & 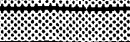 & & $1 \mathrm{H}$ & & \\
\hline $111-\mathrm{T}$ & & & & MIIF & & & & $\mathrm{NT}$ & & \\
\hline $112-T$ & & & & 46 & 4 & $4 \%$ & 14 & 14 & & \\
\hline $201-T$ & & & & & TIIS & $10 \%$ & 610 & $10 \pi$ & & \\
\hline $202-T$ & & & & & 14 & 84 & $4 \%$ & 4 & & \\
\hline $203-T$ & & & & & $1 \%$ & $1 \mathrm{m \alpha}$ & $\alpha \%$ & NT & & \\
\hline $204-T$ & & & & & 14 & $4 \alpha$ & 4 & 10 & & \\
\hline $101-T X$ & & & OIS - O/C & N2 & & 40 & NIS & NOA & & \\
\hline 102-TX & & & O/C & & Wy & & & NH & & \\
\hline $103-T X$ & & & O/C & MNO & & 8078 & $8 \pi$ & $\mathrm{NT}$ & & \\
\hline 104-TX & & & O/C & $r y$ & & 4 & $4 \alpha$ & 4 & & \\
\hline 105-TX & $\bar{x}$ & & & & 144 & O/S-O/C & O/SOIC & $\mathrm{Nr}$ & & \\
\hline 106-TX & & & $\mathrm{OKC}$ & & 14 & & & $1+1$ & & \\
\hline 107-TX & & & OIC & INI & & $8 \mathrm{NI}$ & $8 \mathrm{Nr}$ & NTr & & \\
\hline 108-TX & & & O/C & 4 & & & & 14 & & \\
\hline $10 \theta-\mathrm{TX}$ & & & O/C & 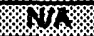 & & & & $\mathrm{NrS}$ & & \\
\hline $110-\mathrm{TX}$ & & & O/C & & 14 & & & $N 4$ & & \\
\hline $111-T X$ & & & O/C & & 121 & & & $\mathrm{~N} / 8$ & & \\
\hline $112-T X$ & & & $\overline{O K C}$ & & 10 & & & $14 \%$ & & \\
\hline $113-T X$ & & & O/C & & $1 \%$ & & & $\mathrm{NrA}$ & & \\
\hline 114-TX & & & O/C & & 14 & & & $\mathrm{~N} / \mathrm{H}$ & & \\
\hline 115-TX & & & O/C & & 14 & & & NTS & & \\
\hline 116-TX & & & O/S O/C & & 10 & 44 & N14 & 10 & & \\
\hline $117-\mathrm{TX}$ & & & O/C & & $1 \%$ & $\mathrm{O} / \mathrm{C}$ & & $\mathrm{NIA}$ & & \\
\hline 118-TX & $\bar{x}$ & & & $\mathrm{NH}$ & & O/C & & 4 & & \\
\hline 101-TY & $\bar{x}$ & & & $1 N 2 \%$ & & $\mathrm{NM}$ & WIA & NTS & & \\
\hline 102-TY & & & & $4 \%$ & & 42 & $4 \mathrm{~A}$ & 14 & & \\
\hline 103-TY & $\bar{x}$ & & & 10 & & O/C & & 1NA & & \\
\hline 104-TY & $\bar{x}$ & & & 110 & & ONT & 101 & NA & & \\
\hline $105-T Y$ & & & & & NT1\% & NTY & 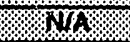 & Nr/ & & \\
\hline $106-\mathrm{TY}$ & & & & & 14 & 41 & 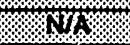 & 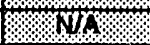 & & \\
\hline $101-U$ & & & & & 19 & 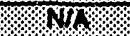 & ont & $\mathrm{NIA}$ & & \\
\hline $102-\mathrm{U}$ & & & & 140 & & & & NHA & & \\
\hline $103-U$ & $\bar{x}$ & & & $112 \%$ & & & & $\mathrm{NA}$ & & \\
\hline 104-U & & & O/S - O/C & & 14 & $4 y$ & 14 & 14 & & \\
\hline $105-U$ & $\bar{x}$ & & & 44 & & & & $\mathrm{NA}$ & & \\
\hline 106-U & $\bar{x}$ & & & 110 & & & & $\mathrm{NA}$ & & \\
\hline
\end{tabular}




\section{TABLE 8. SINGLE-SHELL TANKS MONITORING COMPLIANCE STATUS 149 TANKS (Sheet 4 of 4 )}

Information as of 12/31/92

\begin{tabular}{|c|c|c|c|c|c|c|c|c|c|c|}
\hline \multirow{4}{*}{$\begin{array}{l}\text { Tank } \\
\text { Number }\end{array}$} & \multirow{2}{*}{\multicolumn{2}{|c|}{ Category }} & \multirow{4}{*}{$\begin{array}{l}\text { Temperature } \\
\text { Readings (5) }\end{array}$} & \multirow{3}{*}{\multicolumn{2}{|c|}{$\begin{array}{c}\text { Surface Level } \\
\text { Readings (1) } \\
\text { (-357) }\end{array}$}} & \multirow{3}{*}{\multicolumn{2}{|c|}{$\begin{array}{l}\text { LOW Roadings } \\
(-357)\end{array}$}} & \multicolumn{2}{|c|}{ Radiation Readings } & \multirow{4}{*}{$\begin{array}{c}\text { Dome } \\
\text { Elevation } \\
\text { Surveys } \\
\text { (OSR/SAR) }\end{array}$} \\
\hline & & & & & & & & \multirow{3}{*}{$\begin{array}{l}\text { Lateral } \\
\text { Readings } \\
(-357)\end{array}$} & \multirow{3}{*}{$\begin{array}{c}\text { Drywell } \\
\text { Readings } \\
\text { (OSR/SAR) }\end{array}$} & \\
\hline & \multirow{2}{*}{$\begin{array}{l}\text { Watch } \\
\text { List }\end{array}$} & \multirow{2}{*}{$\begin{array}{l}\text { High } \\
\text { Heat }\end{array}$} & & & & & & & & \\
\hline & & & & M.T. & FIC & Gamma & Neutron & & & \\
\hline $107-U$ & $\overline{\bar{x}}$ & & & m & & & & 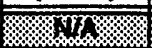 & & \\
\hline $108-U$ & $\bar{x}$ & & & 4 & & & & 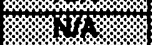 & & \\
\hline $100-U$ & $\bar{x}$ & & & $51 \%$ & & & & 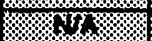 & & \\
\hline $110-U$ & & & & 14 & & 4 & 4 & 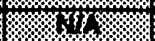 & & \\
\hline $111-U$ & & & & 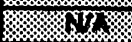 & & & & 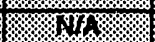 & & \\
\hline $112-U$ & & & & & 4 & $4 \%$ & $1 \%$ & 4 & & \\
\hline $201-U$ & & & & & 10 & 10 & r.s. & m & & \\
\hline $202-U$ & & & & & 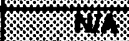 & 4 & 1 & 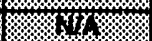 & & \\
\hline $203-U$ & & & OrC & & 8 & 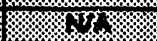 & 8178 & \%1\% & & \\
\hline $204-U$ & & & O/C & & $\%$ & 13 & 46 & 4 & & \\
\hline \multicolumn{11}{|c|}{ Catch Tanks } \\
\hline$A-302-A$ & N/A & N/A & 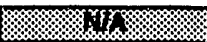 & res & & 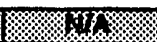 & rar & Xr. & Wro & Mrf \\
\hline$A-302-B$ & N/A & N/A & $8 \mathrm{H}$ & & $8 \%$ & $\%$ & Wto & $4 \pi$ & $\alpha$ & Nor \\
\hline 311-ER & N/A & N/A & or & Yr. & & 13 & 10 & oro & Not7 & Nor \\
\hline Vent Sta. & NA & N/A & 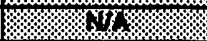 & & 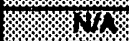 & $\%$ & 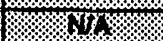 & Nith & $\pi$ & Sur \\
\hline $151-A Z$ & N/A & N/A & SWr & 10 & & mo & $r r$ & gir & Nors & sto \\
\hline 154-AZ & N/A & N/A & $14 \%$ & & $10 \%$ & $4 x$ & 4 Wh & Nut & ovis & ont \\
\hline S-302 & NA & N/A & $\%$ & YY & & rys & rer & Wro & Nor & $8 \times$ \\
\hline$S-302-A$ & N/A & N/A & 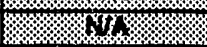 & $11 \%$ & $\mathrm{O} / \mathrm{C}$ & 21 & 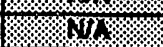 & $14 \%$ & $10 \%$ & 14 \\
\hline$T X-302-B$ & N/A & NA & 10 & OKC & YY\% & 192 & 10 & sots & 1018 & $8 \%$ \\
\hline$T X-302-C$ & NA & N/A & NOK & 82 & & 10 & 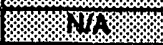 & 40 & NII & $0 \%$ \\
\hline$U-301-B$ & NA & N/A & 8 & $8 \%$ & O/C & sy & Nor & X19. & rer & \$NA \\
\hline$U X-302-A$ & N/A & NA & $\mathrm{N} /$ & 114 & & N1\% & surs & $\mathrm{NTS}$ & Nus & $\mathrm{NrK}$ \\
\hline 141-S & N/A & $\mathrm{N} / \mathrm{A}$ & mrs & & YT. & ror. & nirs & in & NorA & $\mathrm{NTS}$ \\
\hline $142-S$ & N/A & N/A & 41 & & 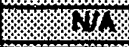 & 4 & $8 \%$ & 0 & $\mathbb{N I A}$ & Nu \\
\hline \multirow{3}{*}{$\begin{array}{l}\text { Totals: } \\
148 \text { tank8 }\end{array}$} & \multirow{3}{*}{$\begin{array}{c}48 \\
\text { Watch } \\
\text { Liet } \\
\text { Tanke } \\
\text { (4) }\end{array}$} & \multirow{3}{*}{$\begin{array}{c}11 \\
\text { High } \\
\text { Heat } \\
\text { Tanks } \\
\text { (4) }\end{array}$} & \multirow{3}{*}{$\begin{array}{l}\text { O/C: } \\
33 \text { tanks - } \\
\text { (eemiannual } \\
\text { monitoring } \\
\text { frequency) (5) }\end{array}$} & \multirow{3}{*}{$\begin{array}{l}\text { OiC: } \\
1 \text { tank }\end{array}$} & \multirow{3}{*}{$\begin{array}{l}\text { O/C: } \\
3 \text { tanks }\end{array}$} & O/C: & O/C: & \multirow{3}{*}{$\begin{array}{l}\text { O/C: } \\
15 \text { tanke }\end{array}$} & \multirow[t]{3}{*}{0} & \multirow[t]{3}{*}{0} \\
\hline & & & & & & 6 tanke & 2 tanks & & & \\
\hline & & & & & & \multicolumn{2}{|c|}{$\begin{array}{l}58 \text { tanks have LOW: } \\
\text { (2 are O/S) }\end{array}$} & & & \\
\hline
\end{tabular}

(1) All SSTs have either manual tape or FIC, with the exception of 108-S which has both. All SST FICs are connected to CASS, (with the exception of 106-BX); however, the connection for many tanks is broken. For such cases, manual field readings are taken. Manual surface level readings include readings taken by manual tape, manual FIC (not connected to Computer Automated Surveillance System, 106-BX), manual readings of automatic FIC (if CASS is printing " $0^{n}$ ), or automatic FIC. In some cases, the surface level readings are taken using a zip cord. While less accurate, such readings are acceptable for meeting the surface level reading requirements.

(2) Psychrometric readings are taken on tanks with active exhausters (High heat tanks 104/105-A, 105/106-C, 107, 108, 109, 110, 111, 112, and 114-SX). Psychrometrics were not taken from 9/91 to 9/92. Exhauster down on 104/105-A since 10/1/91. 105/106-C have resumed psychrometric readings on a monthly frequency. Psychrometric frequency not specified in -357 or operating procedures.

(3) In-tank Photographs are not required by -357. Last in-tank photographs in SSTs were taken September 1990.

(4) Two tanks are on both category lists (106-C, 109-SX).

(5) Temperature readings may be regulated by OSD, -357, or POP. Additionally, high heat load tanks are regulated by OSP/SAR. Thermcouples in the 9 tanks designated O/S-O/C are out of service; there are either no thermocouple probes in these tanks, or probes have been cut off, covered over, or are otherwise not functioning. The OSD does not require readings or repair of out-of-service thermocouples for non-heat load $(>40,000 \mathrm{Btu} / \mathrm{hr})$ tanks. However, the operating procedure requires that attempts are to be made semiannually in January and July to obtain readings from these tanks. The remaining 24 tanks are O/C because they had readings at the semiannual date of January 1992 but no readings since that time. 


\section{WHC-EP-0182-57 \\ TABLE 9. DOUBLE-SHELL TANKS MONITORING COMPLIANCE STATUS 28 TANKS}

The following table indicates whether Double-Shell tank monitoring is in compliance with the requirements as specified in the applicable documents:

NOTE:

All DST tank temperature tree monitoring is in compliance.

Dome Elevation Surveys are not required for DSTs.

Psychrometrics (2)

In-tank Photographs (3)

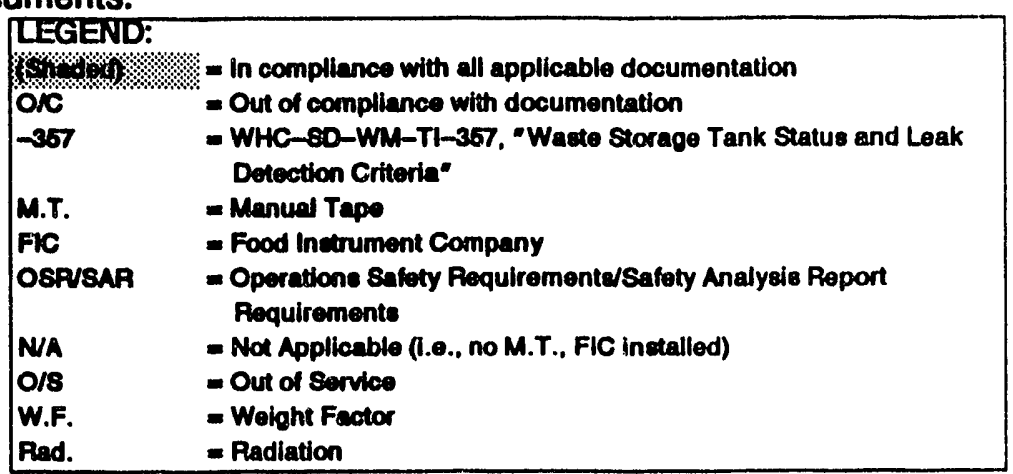

Information as of $12 / 31 / 92$

\begin{tabular}{|c|c|c|c|c|c|c|c|}
\hline \multirow{3}{*}{$\begin{array}{l}\text { Tank } \\
\text { Number }\end{array}$} & \multirow[b]{3}{*}{ Watch List } & \multirow{3}{*}{$\begin{array}{l}\text { Temperature } \\
\text { Readings } \\
\text { (4) } \\
\text { (OSASAR) }\end{array}$} & \multirow{2}{*}{\multicolumn{2}{|c|}{$\begin{array}{l}\text { Surface Level } \\
\text { Readings (1) } \\
\text { (-357, OSR/SAR) }\end{array}$}} & \multicolumn{3}{|c|}{ Radiation Readings } \\
\hline & & & & & \multicolumn{2}{|c|}{$\begin{array}{c}\text { Leak Detection } \\
\text { Pits (5) } \\
\text { (-357, OSR/SAR) }\end{array}$} & \multirow{2}{*}{$\begin{array}{c}\text { Annulus } \\
(-357)\end{array}$} \\
\hline & & & M.T. & $F I C$ & W.F. & Rad. & \\
\hline $101-A N$ & & & $1 \%$ & & & & \\
\hline 102-AN & & & 10\% & & & OIC & \\
\hline 103-AN & $\bar{x}$ & & YW & & & & \\
\hline $104-A N$ & $\bar{x}$ & & m1 & & & & \\
\hline $105-A N$ & $\bar{x}$ & & 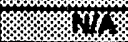 & & & & \\
\hline $106-A N$ & & & $120 \%$ & & & & \\
\hline $107-A N$ & & & $1 \times 1 \%$ & & & & \\
\hline 101-AP & & & & & & O/S & \\
\hline 102-AP & & & & & & O/S & \\
\hline 103-AP & & & & & & O/S & \\
\hline 104-AP & & & & & & O/S & \\
\hline 105-AP & & & & $\%$ & & O/S & \\
\hline 106-AP & & & & & 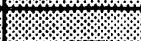 & O/S & \\
\hline 107-AP & & & & & & O/S & \\
\hline 108-AP & & & & $\$$ & $\%$ & O/S & \\
\hline $101-A W$ & $\bar{x}$ & & & & & & \\
\hline $102-A W$ & & & & o/s & & & \\
\hline 103-AW & & & & o/s & & & \\
\hline \multicolumn{8}{|l|}{ 104-AW } \\
\hline \multicolumn{8}{|l|}{ 105-AW } \\
\hline \multicolumn{8}{|l|}{$106-A W$} \\
\hline $101-A Y$ & & & & $0 / 5$ & & & $0 / C$ \\
\hline 102-AY & & & & & & & O/C \\
\hline $101-A Z$ & & & & o/s & m & & O/C \\
\hline $102-A Z$ & & & & o/s & & & O/C \\
\hline 101-SY & $\bar{x}$ & & & & 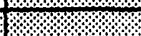 & O/C & \%क \\
\hline $102-5 Y$ & & & & O/S & & & \\
\hline $103-S Y$ & $\bar{x}$ & & & & & OIC & \% \\
\hline $\begin{array}{l}\text { Totals: } \\
28 \text { tanks }\end{array}$ & $\begin{array}{c} \\
\text { Watch Liat Tanke }\end{array}$ & $\begin{array}{l}\text { O/C: } \\
0 \text { (4) }\end{array}$ & $\begin{array}{l}\text { ONe: } \\
0\end{array}$ & $\begin{array}{l}\text { OK: } \\
0\end{array}$ & $\begin{array}{l}\text { O/C: } \\
0\end{array}$ & $\begin{array}{l}\text { O/C: } \\
3 \text { tanks }\end{array}$ & $\begin{array}{l}\text { O/C: } \\
4 \text { tanks }\end{array}$ \\
\hline
\end{tabular}

(1) All DSTs have both manual tape and FIC, with the exception of the AN Tank Farm which only has FICs. The manual tape is used when the FIC is out of service. O/C will be shown when no readings are obtained.

(2) Psychrometric readings are taken on DSTs. All DSTs are equipped with active exhausters. Psychrometrics were not taken from 9/91 to 9/92; readings on some tanks were resumed beginning October 1992. Frequency of psychrometric monitoring is not specified in -357 or operating procedures.

(3) In-tank photographs are not required by -357. Last in-tank photographs in DSTs were taken April 1989.

(4) DST temperature tree readings are taken weekly, with the exception of 101-SY which are obtained shiftwise with increased readings taken prior to and following gas venting.

(5) Failure of both leak detection systems requires repair of at least one system within 5 working days. Failure of one system only, repair must be within 10 working days. Per -357. If repair of out-of-service systems exceeds these timeframes, systems are O/C. Out-of-service systems which have not exceeded these timeframes will be shown as O/S. 
WHC-EP-0182-57

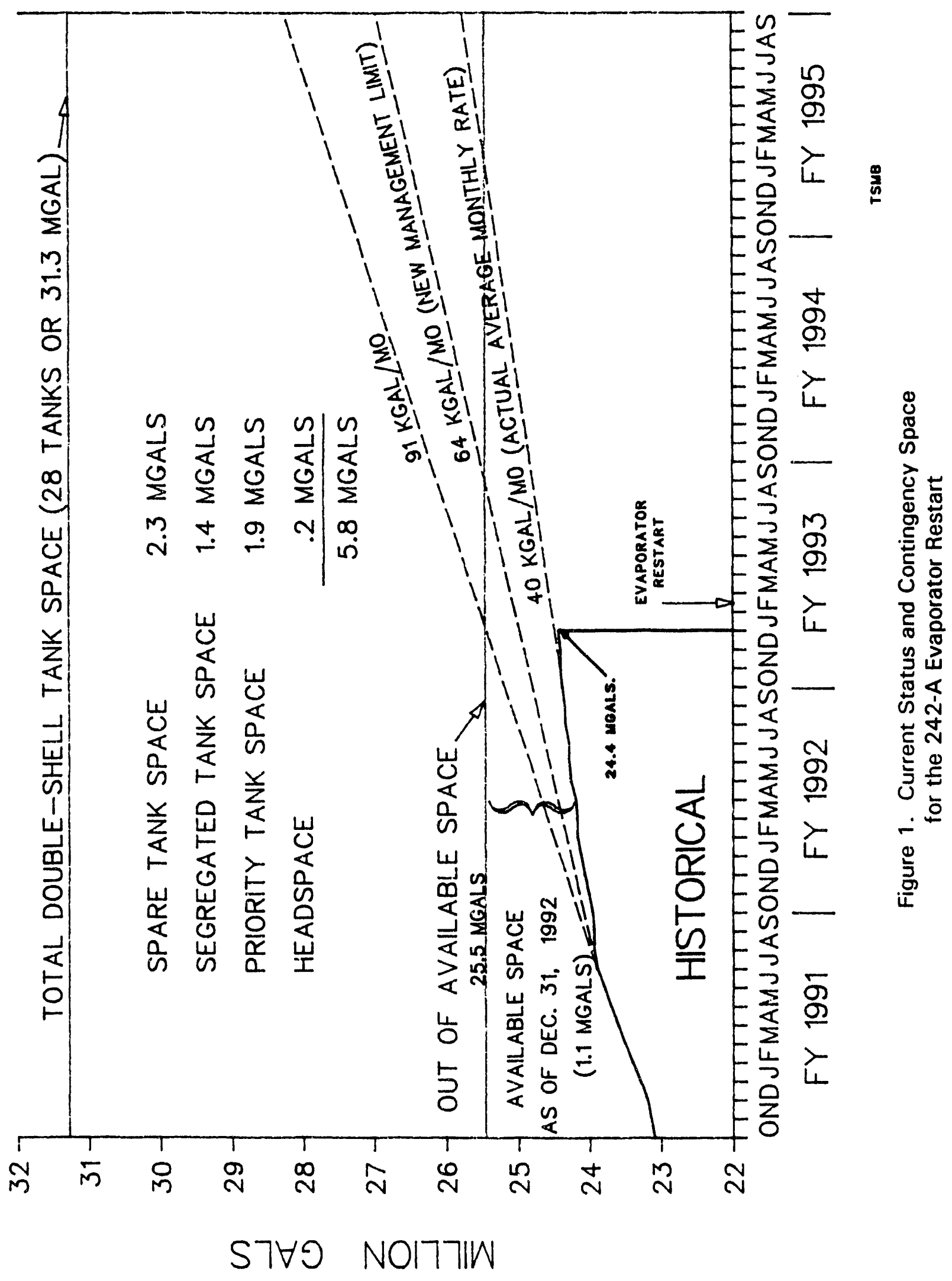


WHC-EP-0182-57

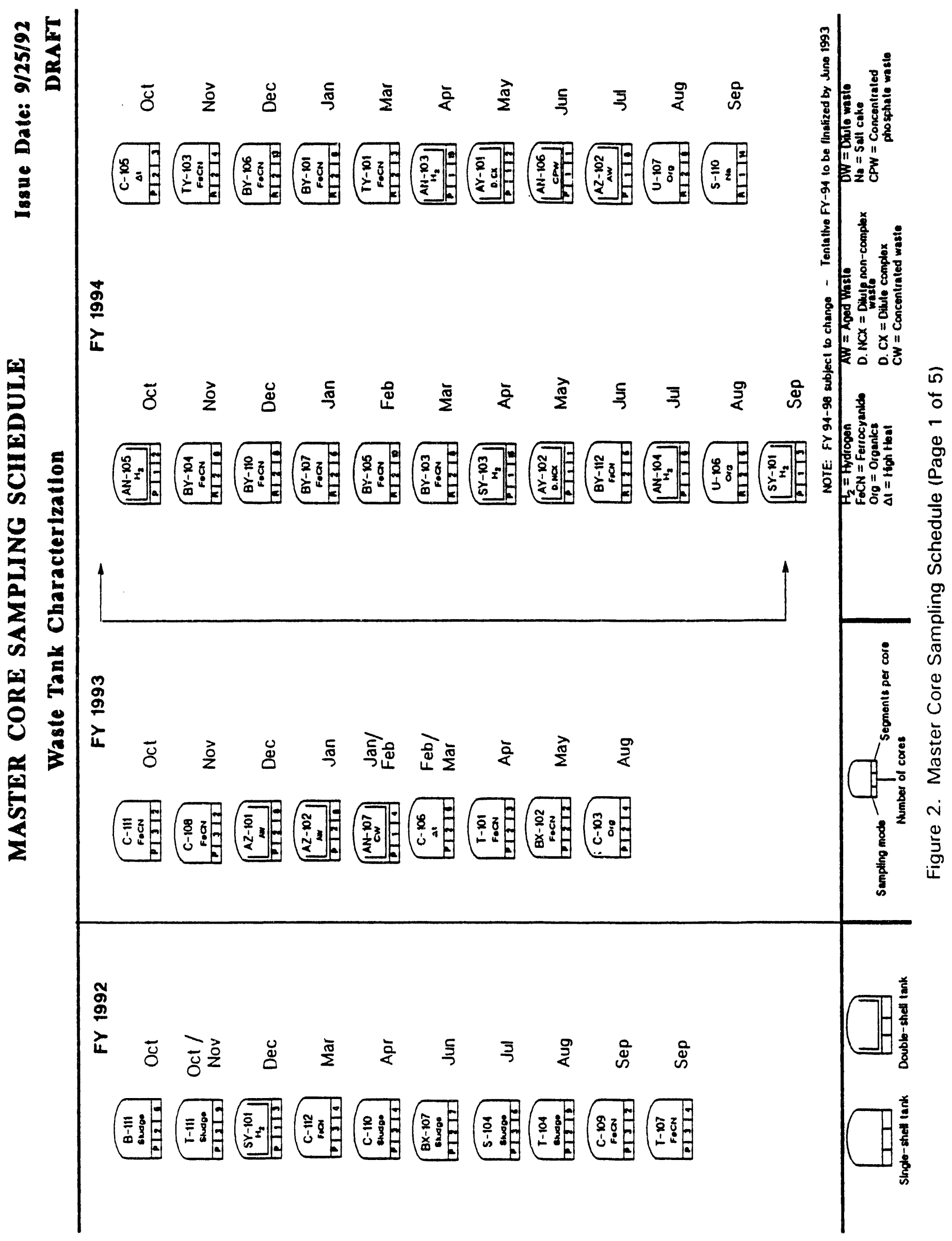


WHC-EP-0182-57
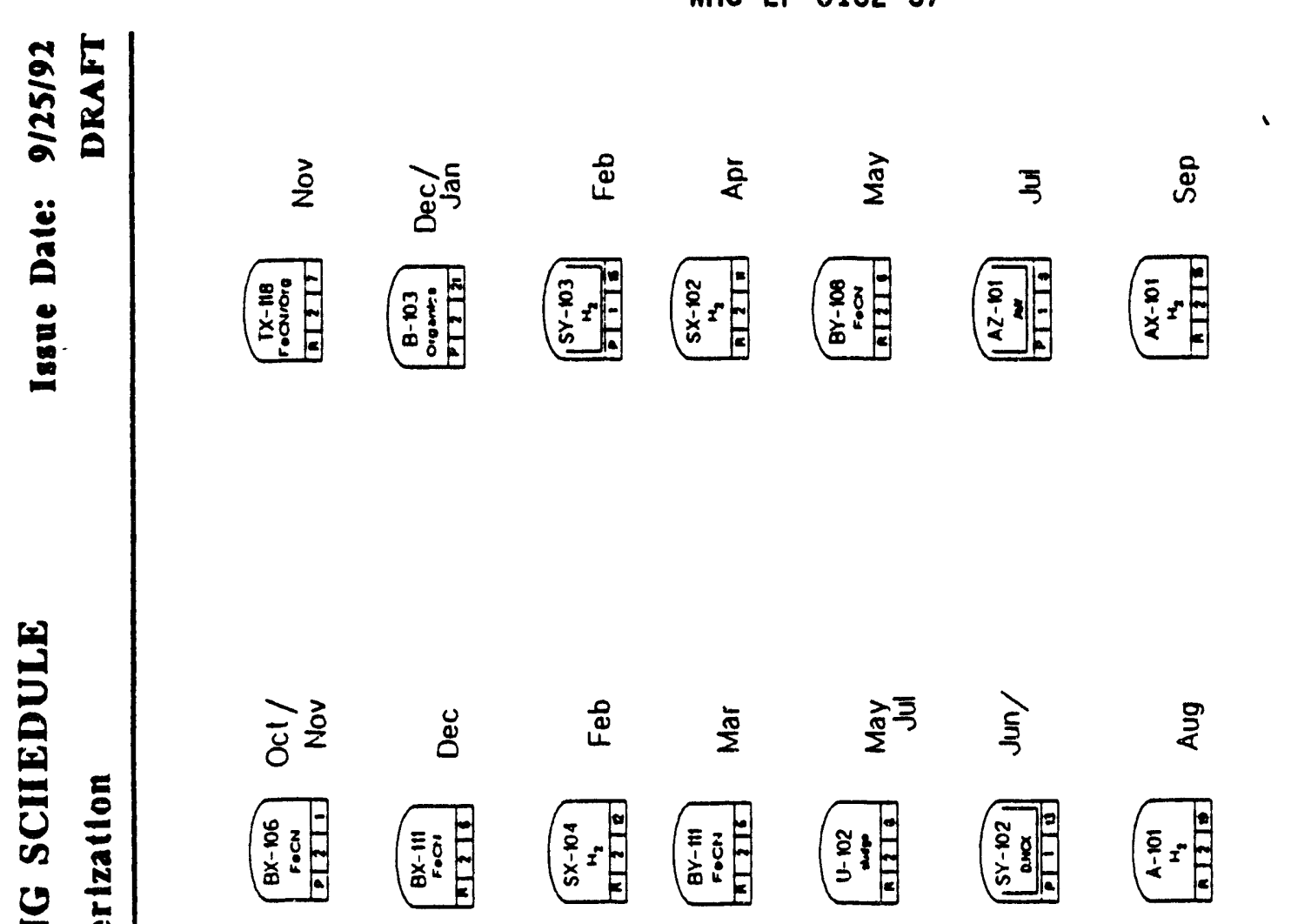
WHC-EP-0182-57

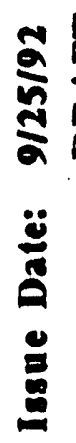

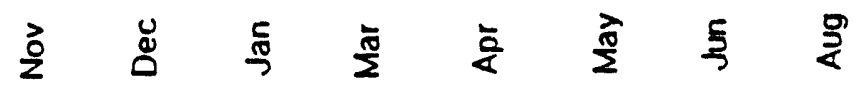

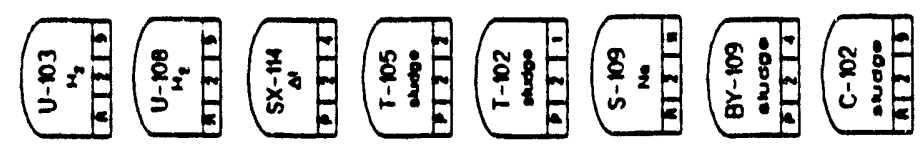

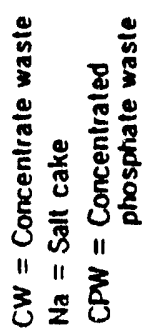

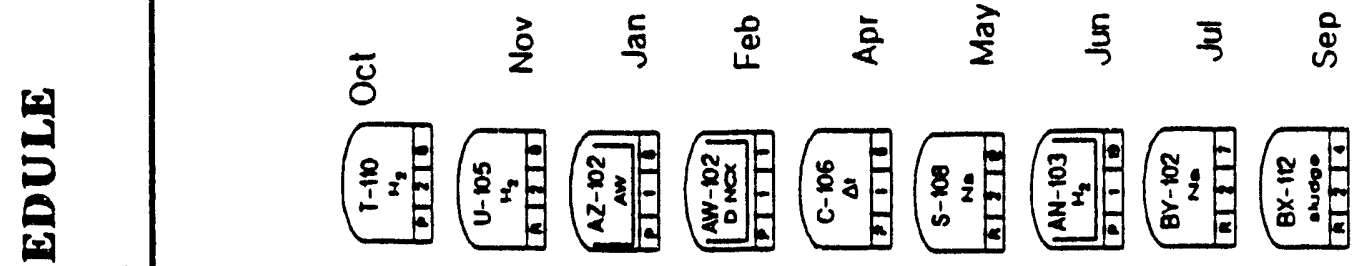

\%

으

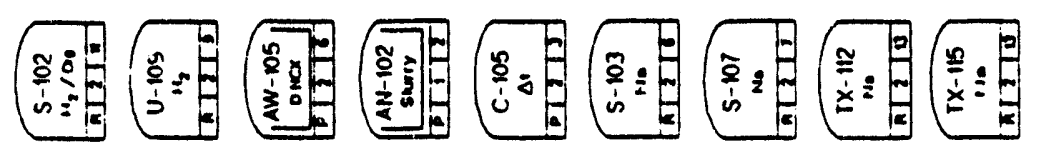

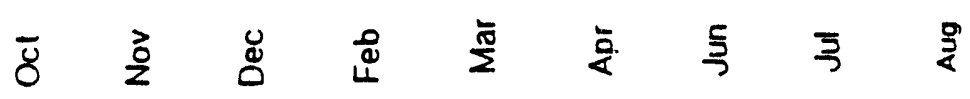

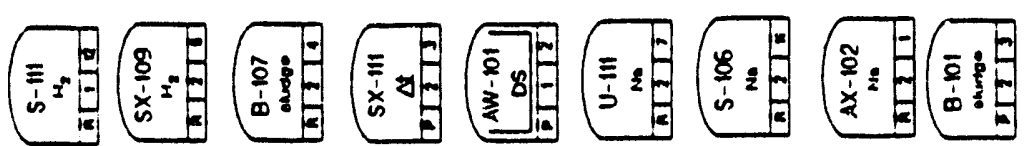

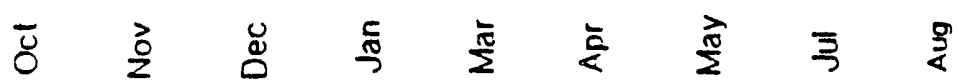

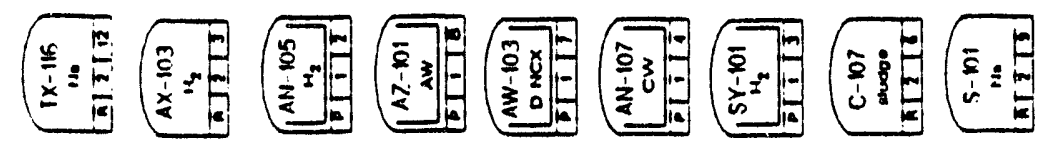

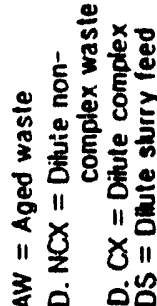

5

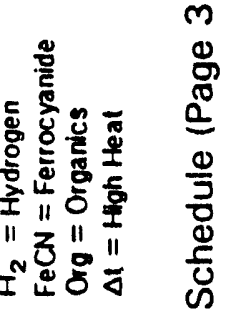

它 产

षे है

产

ॐ

㟧

$\sim 2$

$\div$

人

总

总

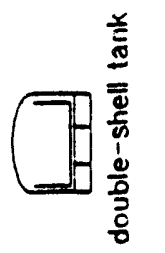

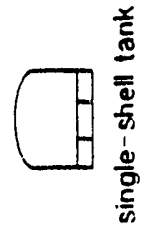


WHC-EP-0182-57

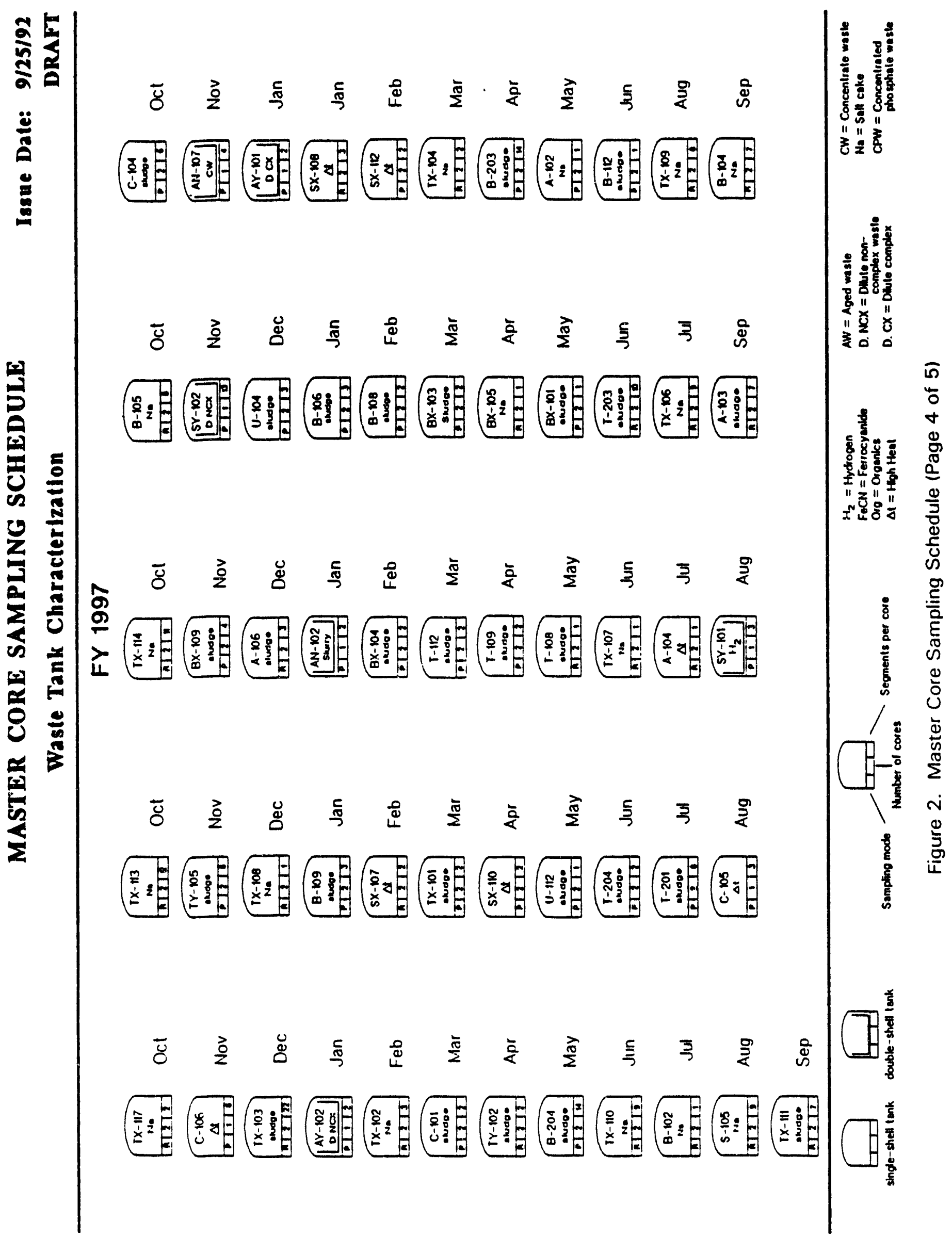


WHC-EP-0182-57

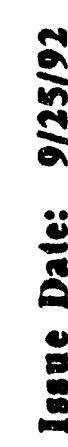

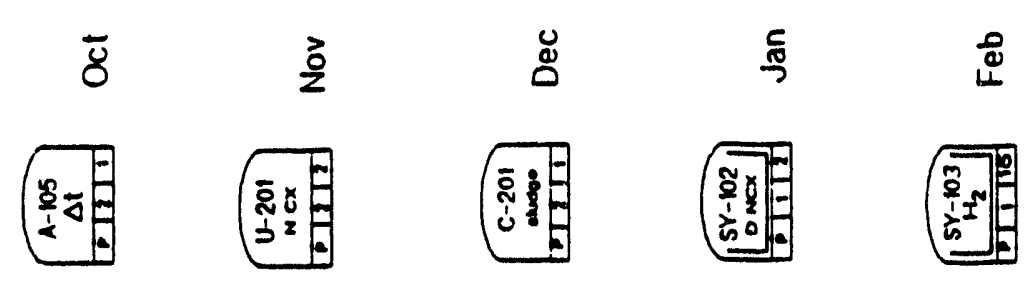

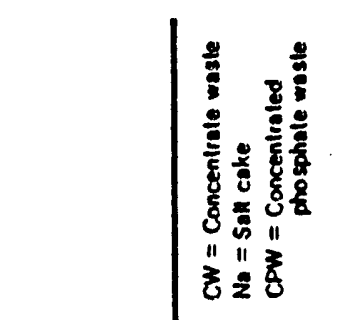

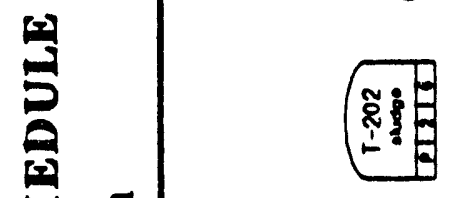

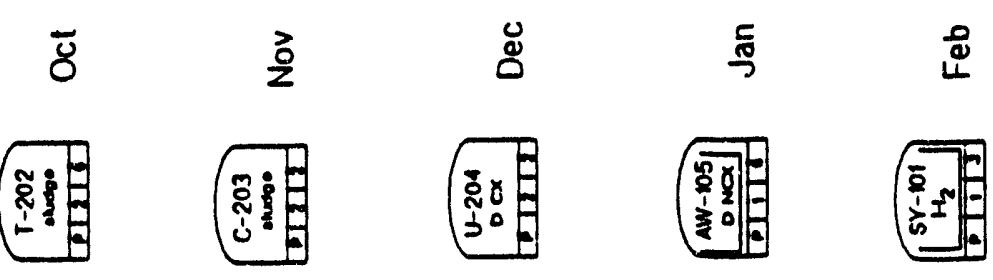

플

恼

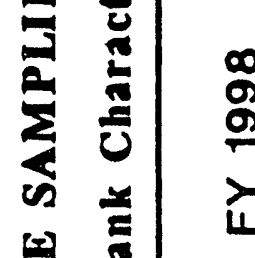

\begin{tabular}{lr}
$\stackrel{\infty}{8}$ & $\overline{8}$ \\
$\stackrel{8}{5}$ & \\
\hline &
\end{tabular}

$\frac{3}{2}$

尊

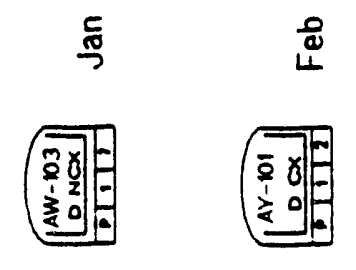

ठำ

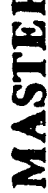

$\overline{8}$

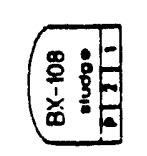

$\stackrel{8}{8} \stackrel{8}{8}$

妾影

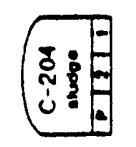

总是

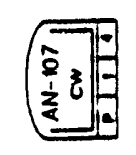

尊是是

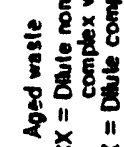

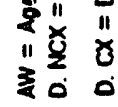

पू

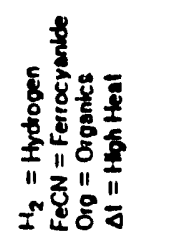

5
5
5
0
8
8
$\frac{0}{0}$
0
3
0
0
0
0

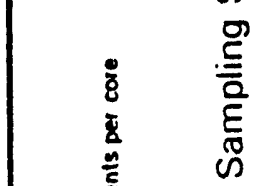

¿ัँ

1

$\frac{5}{2}$

$>\frac{1}{2}$

$\overline{8}$

¿্口

ơ

孚

尊苛

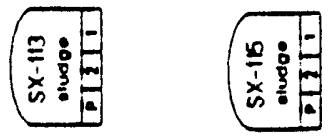

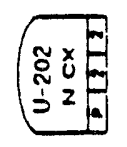

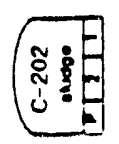

包

家是昌

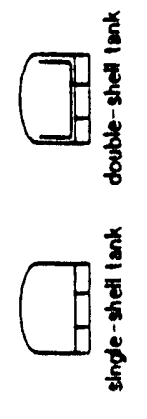


WHC-EP-0182-57

\section{APPENDIX A}

TANK AND EQUIPMENT CODE AND STATUS DEFINITIONS

A-1 
WHC-EP-0182-57

This page intentionally lift biank

A-2 
TANK AND EQUIPMENT CODE/STATUS DEFINITIONS

December 1992

\section{TANK STATUS CODES}

\section{WASTE TYPE}

$\begin{array}{ll}\text { AGING } & \text { Aging Waste (Neutralized Current Acid Waste [NCAW]) } \\ \text { CC } & \text { Complexant Concentrate Waste } \\ \text { CP } & \text { Concentrated Phosphate Waste } \\ \text { DC } & \text { Dilute Complexed Waste } \\ \text { DN } & \text { Dilute Non-Complexed Waste } \\ \text { DSS } & \text { Double-Shell Slurry } \\ \text { DSSF } & \text { Double-Shell Slurry Feed } \\ \text { NCPLX } & \text { Non-Complexed Waste } \\ \text { PD/PN } & \text { Plutonium-Uranium Extraction (PUREX) Neutralized Cladding } \\ & \text { Removal Waste (NCRW) transuranic Waste (TRU) } \\ \text { PT } & \text { Plutonium Finishing Plant (PFP) TRU Solids }\end{array}$

\section{TANK USE (DOUBLE-SHELL TANKS ONLY)}

$\begin{array}{ll}\text { CWHT } & \text { Concentrated Waste Holding Tank } \\ \text { DRCVR } & \text { Dilute Receiver Tank } \\ \text { EVFD } & \text { Evaporate Feed Tank } \\ \text { GRTFD } & \text { Grout Feed Tank } \\ \text { SRCVR } & \text { Slurry Receiver Tank }\end{array}$

2. SOLID AND LIOUID VOLUME DETERMINATION METHODS
$\mathrm{F}$
M
Food Instrument Company (FIC) Automatic Surface Level Gauge
P
Manual Tape Surface Level Gauge
Photo Evaluation
S Sludge Level Measurement Device

\section{DEFINITIONS}

WASTE TANKS - GENERAL
Waste Tank
Safety Issue
A potentially unsafe condition in the handling of waste material in underground storage tanks that requires corrective action to reduce or eliminate the unsafe condition
Watch List Tank
An underground storage tank containing waste that requires special safety precautions because it may have a serious potential for release of high level radioactive was because of uncontrolled increases in temperature or pressure. Special restrictions have been placed on these tanks by "Safety Measures for Waste Tanks at Hanford Nuclear Reservation, Section 3137 of the National Defense Authorization Act for Fiscal Year 1991, November 5, 1990, Public Law 101- 510, (also known as the Wyden Amendment). 
WASTE TYPES

Aging Waste

(AGING)

Concentrated

Complexant (CC)

Concentrated

Phosphate Waste

(CP)

Dilute Complexed Waste (DC)
High level, first cycle solvent extraction waste from the PUREX plant (NCAW)

Concentrated product from the evaporation of dilute complexed waste.

Waste originating from the decontamination of $100 \mathrm{~N}$ Area reactor. Concentration of this waste produces concentrated phosphate waste.

Characterized by a high content of organic carbon including organic complexants:

ethylenediaminetetra-acetic acid (EDTA), citric acid, hydroxyethyl-ethylenediaminetriacetic acid (HEDTA), and iminodiacetate (IDA) being the major complexants used. Main sources of DC waste in the DST system are saltwe 11 1 iquid inventory.

Dilute Non-Complexed Waste (DN)

Low activity liquid waste originating from $T$ and $S$ Plants, the 300 and 400 Areas, PUREX facility (decladding supernatant and miscellaneous wastes), $100 \mathrm{~N}$ Area (sulfate waste), B Plant, saltwells, and PFP (supernate).

Double-Shell

Siurry (DSS)

Double-Shel1

Slurry Feed (DSSF)

Non-complexed

(NCPLX)

PUREX Decladding $(\mathrm{PD} / \mathrm{PN})$

PFP TRU Solids (PT)

Drainable

Interstitial

Liquid (DIL)

Supernate
Waste that exceeds the sodium aluminate saturation boundary in the evaporator without exceeding receiver tank composition limits. For reporting purposes, DSS is considered a solid.

Waste concentrated just before reaching the sodium aluminate saturation boundary in the evaporator without exceeding receiver tank composition limits. This form is not as concentrated as DSS.

General waste term applied to all Hanford site liquors not identified as complexed.

PUREX Neutralized Cladding Removal Waste (NCRW) is the solids portion of the PUREX plant neutralized cladding removal waste stream; received in Tank Farms as a slurry. NCRW solids are classified as transuranic (TRU) waste.

TRU solids fraction from PFP Plant operations.

Interstitial liquid that is not held in place by capillary forces, and will therefore migrate or move by gravity.

The liquid above the solids in waste storage tanks. 
Ferrocyanide

WASTE STATUS

In-Service Tank

Out-of-Service

Tank
A compound of iron and cyanide commonly expressed as FeCN. The actual formula for the ferrocyanide anion is $\left[\mathrm{Fe}(\mathrm{CN})_{6}\right]^{-4}$.

The waste classification of a tank being used, or planned for use, for the storage of liquid (in excess of a minimum supernatant liquid heel) in conjunction with production and/or waste processing.

A tank which does not meet the definition of an inservice tank. Before September 1988, these tanks were defined as inactive in this report. [Note: All single-shell tanks (SST) are out of service.]

STABILIZATION (Single-Shell Tanks only)

Interim

Stabilized

(IS)
A tank which contains less than 50,000 gal of drainable interstitial liquid and less than 5,000 gal of supernatant liquid. If the tank was jet pumped to achieve interim stabilization, then the jet pump flow must also have been at or below $0.05 \mathrm{gpm}$ before interim stabilization criteria is met.

\section{ISOLATION (Single-Shell Tanks only)}

Partially

Interim Isolated

(PI)

Interim Isolated (II)

\section{TANK INTEGRITY}

Sound

Assumed Leaker

\section{TANK INVESTIGATION}

Intrusion
The administrative designation reflecting the completion of the physical effort required for Interim Isolation except for isolation of risers and piping that is required for jet pumping or for other methods of stabilization.

The administrative designation reflecting the completion of the physical effort required to minimize the addition of liquids into an inactive storage tank, process vault, sump, catch tank, or diversion box.

The integrity classification of a waste storage tank for which surveillance data indicate no loss of liquid attributed to a breach of integrity.

The integrity classification of a waste storage tank for which surveillance data indicate a loss of liquid attributed to a breach of integrity.

A term used to describe the infiltration of liquid into a waste tank. 


\section{SURVEILLANCE INSTRUMENTATION}

Drywells

Laterals

Surface Levels

Automatic FIC

Annulus
Drywells are vertical boreholes with 6 -in. (internal diameter) carbon steel casings positioned radially around SSTs. Periodic monitoring is done by gamma radiation or neutron sensors to obtain scan profiles of radiation or moisture in the soil as a function of well depth, which could be indicative of tank leakage. These wells range between 50 and $250 \mathrm{ft}$ in depth, and are monitored between the range of 50 to $150 \mathrm{ft}$. The wells are sealed when not in use. They are called drywells because they do not penetrate to the water table and are therefore usually "dry." The drywell frequency monitoring schedule calls for 105 drywells weekly, 91 biweekly, 41 monthly, 151 quarterly, and 371 annually.

Laterals are horizontal drywells positioned under single-shell waste storage tanks to detect radionuclides in the soil which could be indicative of tank leakage. These drywells are monitored by radiation detection probes. Laterals are 4-in. inside diameter steel pipes located 8 to $10 \mathrm{ft}$ below the tank's concrete base. There are three laterals per tank. Laterals are located only in $A$ and $S X$ farms.

The surface level measurements in all waste storage tanks are monitored by manual or automatic conductivity probes, and recorded and transmitted or inputted to the Computer Automated Surveillance System (CASS).

An automatic waste surface level measurement device is manufactured by the Food Instrument Company (FIC). The instrument consists of a conductivity electrode (plummet) connected to a calibrated steel tape, a steel tape reel housing and a controller that automatically raises and lowers the plummet to obtain a waste surface level reading. The controller can provide a digital display of the data and also transmit the reading to the CASS. Some tanks have gauges connected to CASS and others are read manualiy.

The annulus is the space between the inner and outer shells on DSTs. Drain channels in the insulating and/or supporting concrete carry any leakage to the annulus space where conductivity probes are installed. Alarms from the annunciators are received by CASS. Continuous Air Monitoring (CAM) alarms are also located in the annulus. The annulus conductivity probes and radiation detectors are the primary means of leak detection for all DSTs. 
Liquid Observation Well (LOW)

Thermocouple (TC)

In-tank

Photography
In-tank liquid observation wells are used for monitoring the interstitial liquid level (ILL) in single-shell waste storage tanks. The wells are constructed of fiberglass, tefzel-reinforced epoxypolyester resin, sized to extend to within 1 in. of the bottom of the tank steel liner. They are sealed at their bottom ends and have a nominal outside diameter of 3.5 in. Three probes are used to monitor changes in the ILL: acoustic; gamma; and neutron, which can indicate intrusions or leakage by increases or decreases in the ILL. There are 58 LOWs (57 are in operation) installed in SSTs that contain or are capable of containing greater than 50,000 gal of drainable interstitial Tiquid, and in two DSTs only. The LOWs installed in two DSTs (102-SY and 103-AW Tanks only) are used for special surveillance purposes only.

A thermocouple is a thermoelectric device used to measure temperature. More than one thermocouple on a device (probe) is called a thermocouple tree. In DSTs there may be one or more thermocouple trees in risers in the primary tank. In addition, in DSTS only, there are thermocouple elements installed in the insulating concrete, the lower primary tank knuckle, the secondary tank concrete foundation, and in the outer structural concrete. These monitor temperature gradients within the concrete walls, bottom of the tank, and the domes. In SSTs, there may be one or more thermocouple trees installed directly in a tank, although some SSTs do not have any trees installed. A single thermocouple may be installed in a riser, or lowered down an existing riser or LOW. There are also four thermocouple laterals beneath Tank 105-A in which temperature readings are taken in 34 thermocouples.

In-tank photographs may be taken to aid in resolving in-tank measurement anomalies and determine tank integrity. Photographs help determine sludge and liquid levels by visual examination.

4. INVENTORY AND STATUS BY TANK - COLUMN CALCULATIONS (SINGLE-SHELL TANKS) COLUMN HEADING

Total Waste Solids Volume plus Supernatant liquid.

Supernatant Drainable Liquid Remaining minus Drainable Interstitial. Liquid Supernate is usually derived by subtracting the solids level measurement from the liquid level measurement.

Drainable Drainable Liquid Remaining minus Supernate. 


\section{WHC-EP-0182-57}

Interstitial Drainable Interstitial Liquid is calculated based on the saltcake and sludge volumes, using average porosity values or actual data for each tank, when available.

Total Jet Pumped

Drainable Liquid

Remaining

Pumpable Liquid

Remaining

Sludge

Cumulative total pumped 1979 to date.

Supernate plus Drainable Interstitial.

Drainable Liquid Remaining less undrainable heel volume.

Saltcake

Solids formed during sodium hydroxide additions to waste. sludge usually was in the form of suspended solids when the waste was originally received in the tank from the waste generator. In-tank photographs may be used to estimate the volume.

Results from crystalization and precipitation after concentration of liquid waste, usually in an evaporator. If saltcake is layered over sludge, it is only possible to measure total solids volume. In-tank photographs may be used to estimate the saltcake volume.

Solids Volume Indicates the latest update of any change in the solids Update volume.

Solids Update Indicates the source or basis of the latest solids Source - See volume update.

Footnote

Last Photo Date of latest in-tank photographs taken.

Date

Change Since Last Monthly Report

Indicates any change made since the previous month. Explanation for the change follows the Inventory and Status by Tank section. 
WHC-EP-0182-57

APPENDIX B

TANK FARM CONFIGURATION, STATUS, AND FACILITY CHARTS 


\section{WHC-EP-0182-57}

This page intentionally left blank. 
WHC-EP-0182
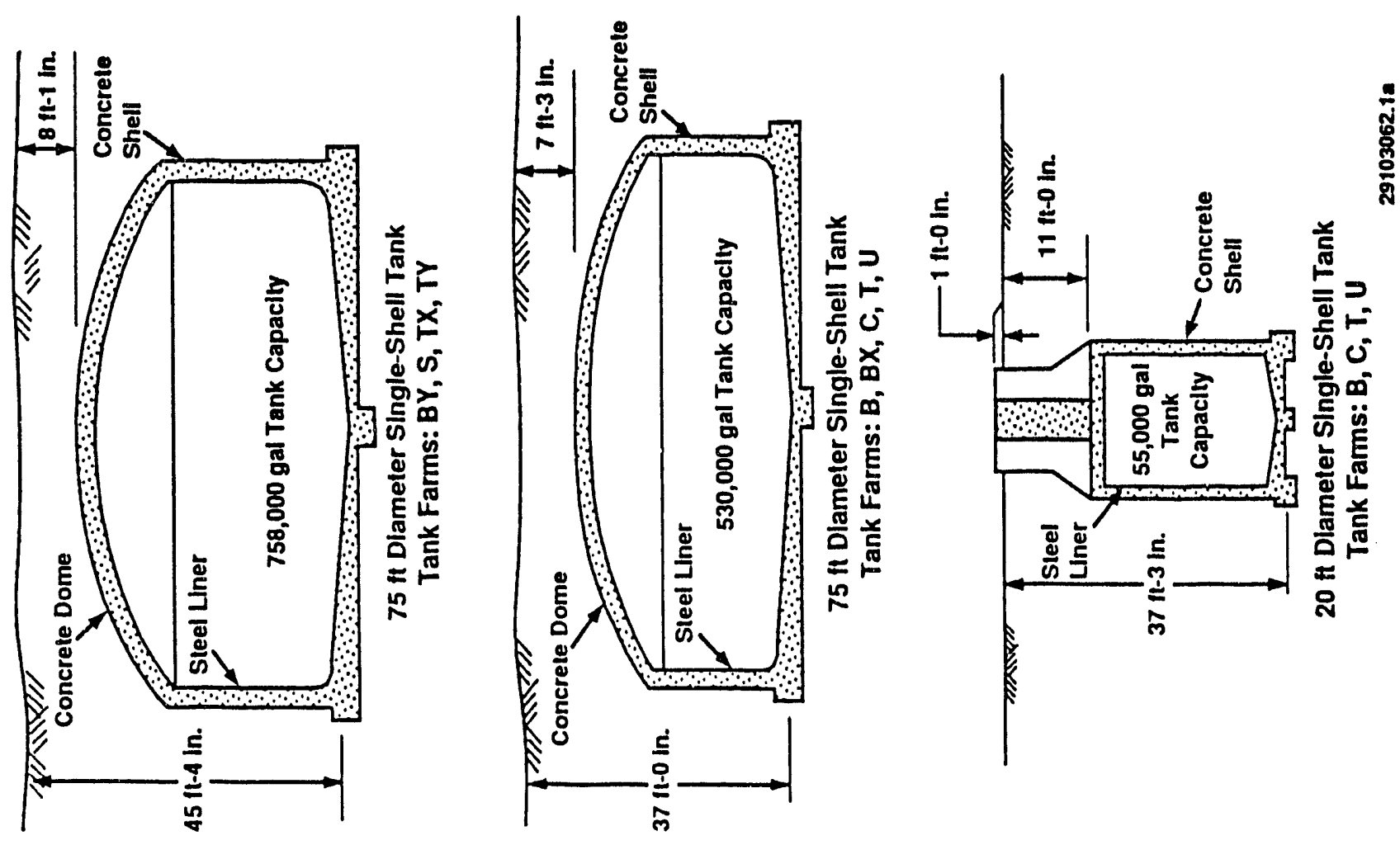

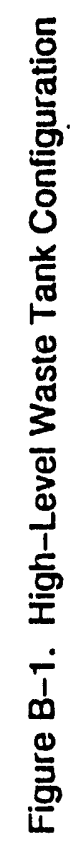
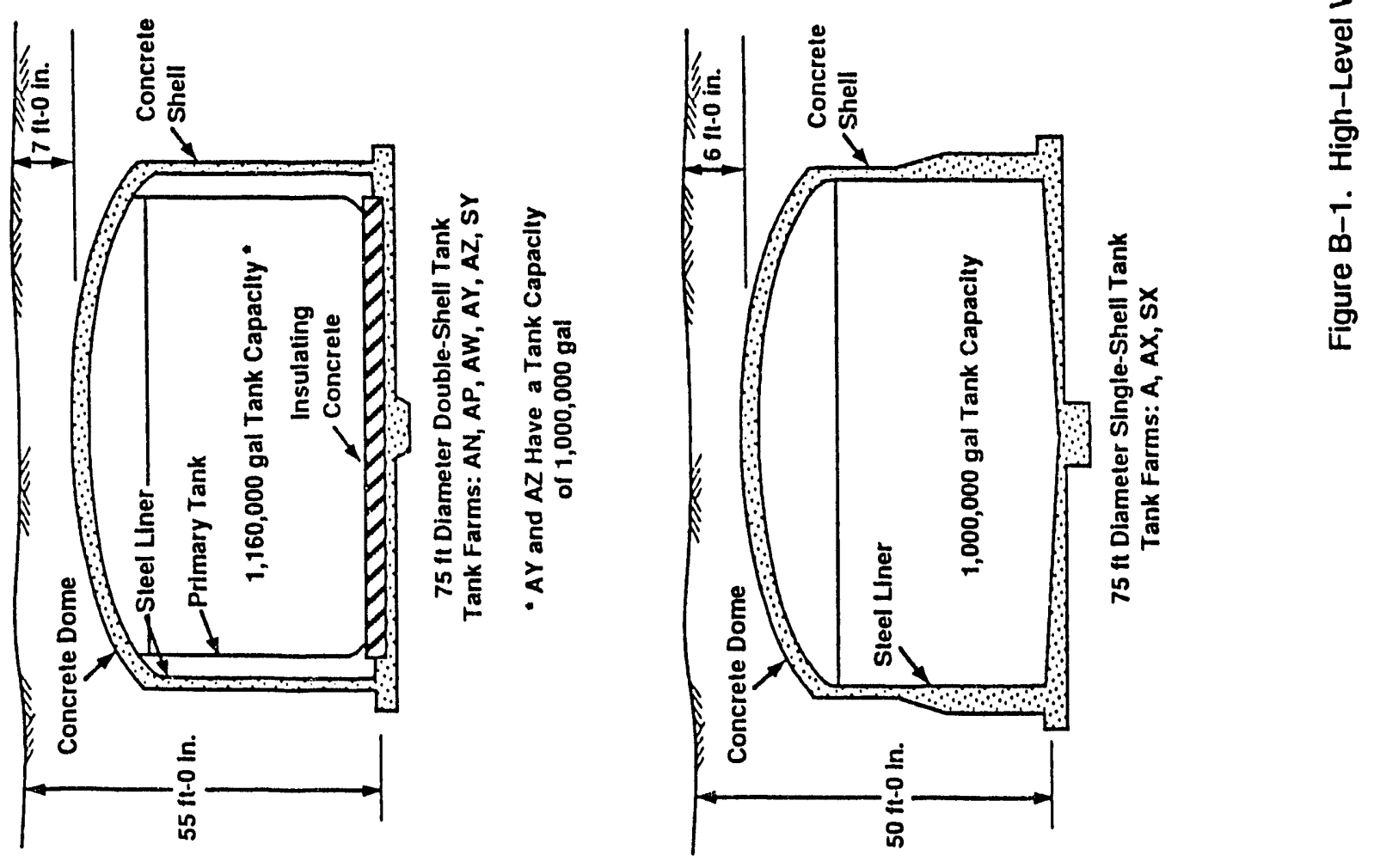


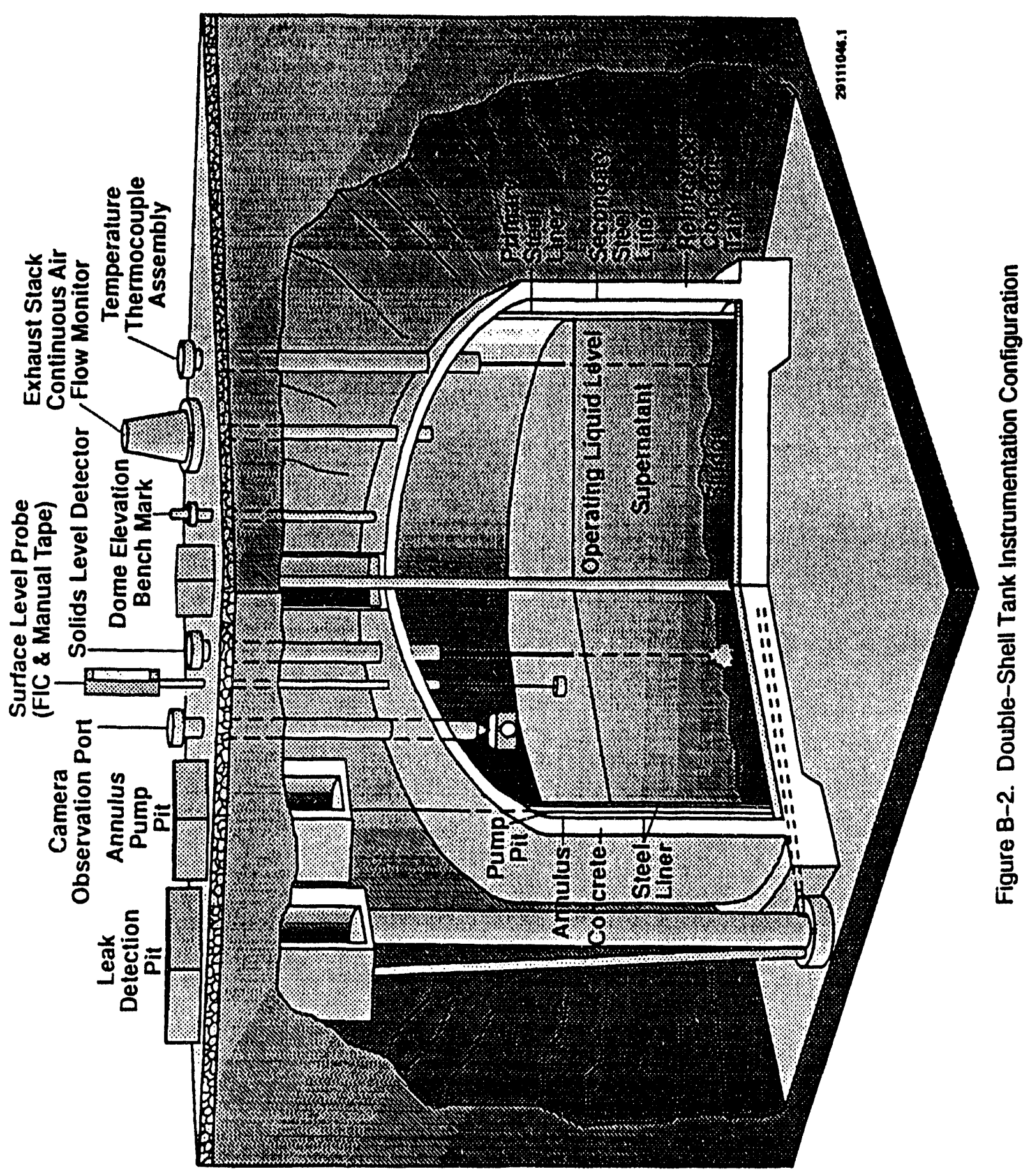




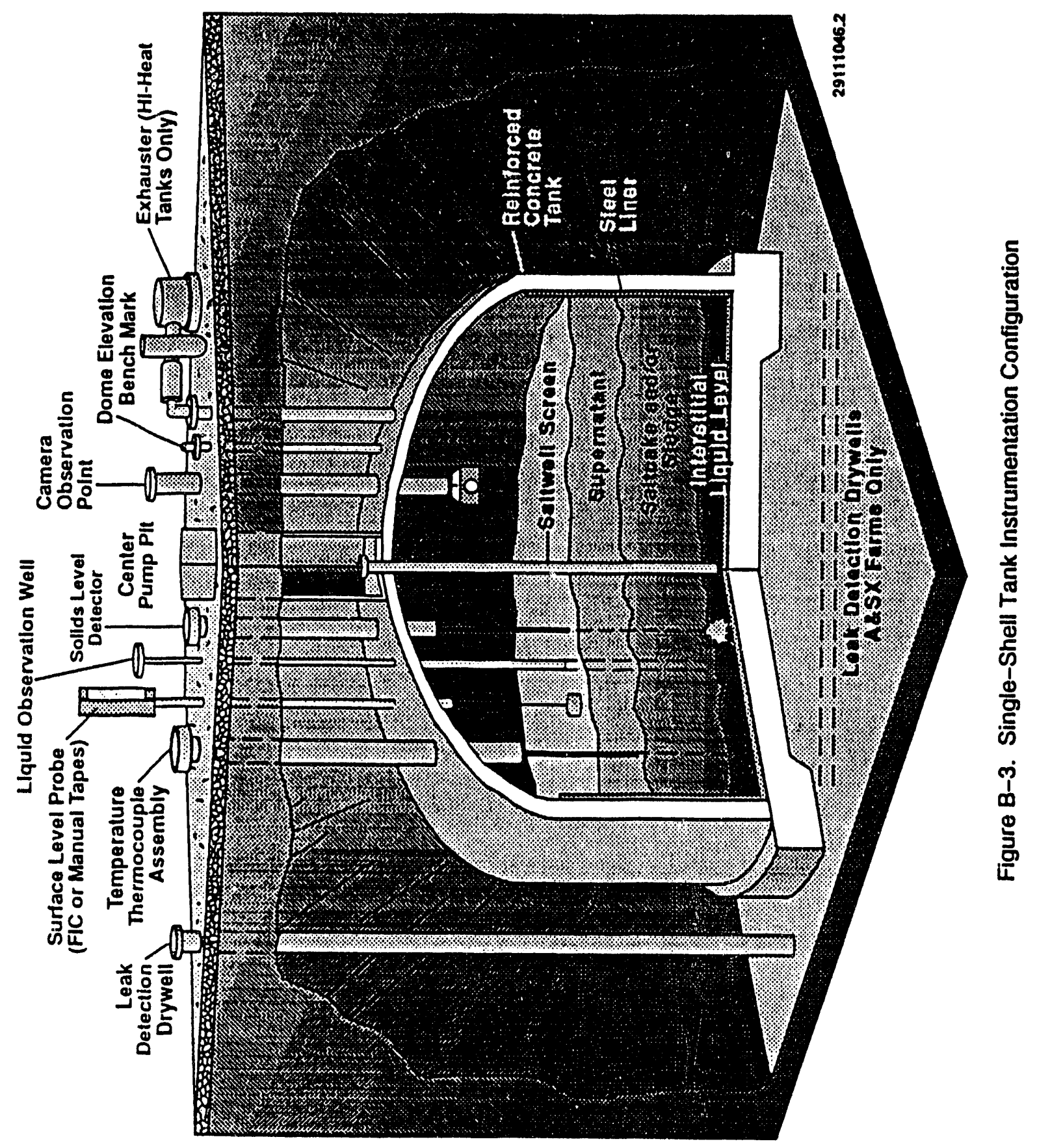




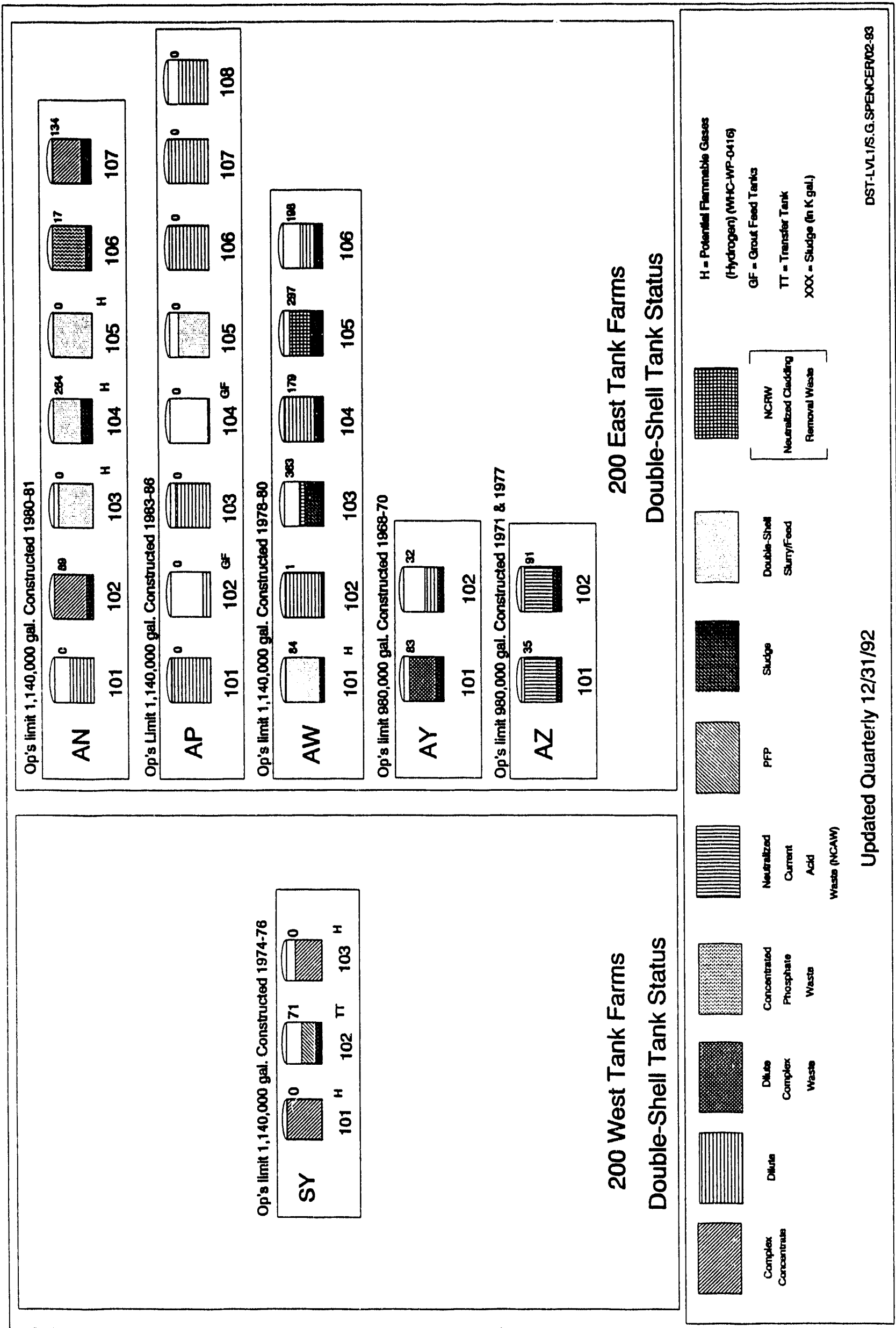


1,000,000 gal. tanks Constructed 1954-55

A

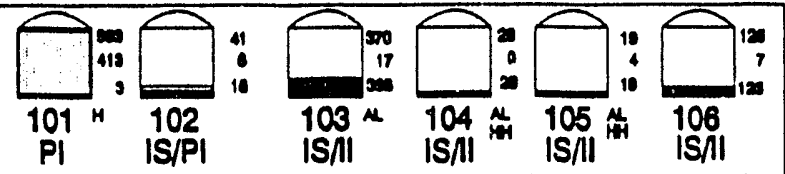

1,000,000 gal. tanks Constructed 1963-64

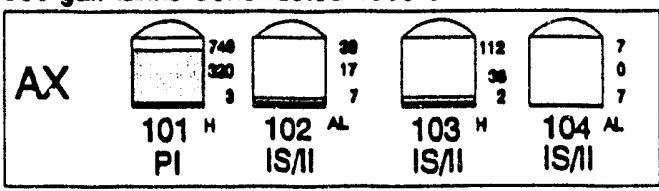

500,000 gal. tanks Constructed 1949-44

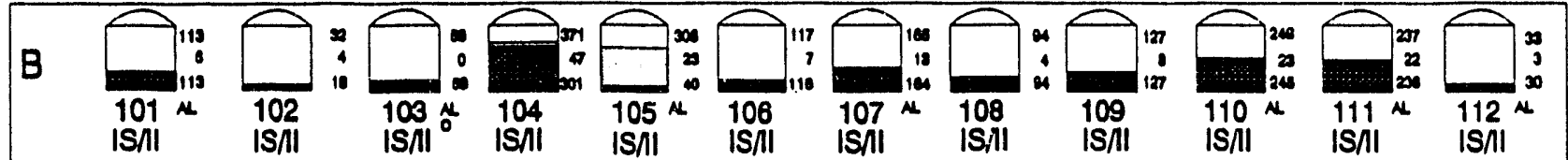

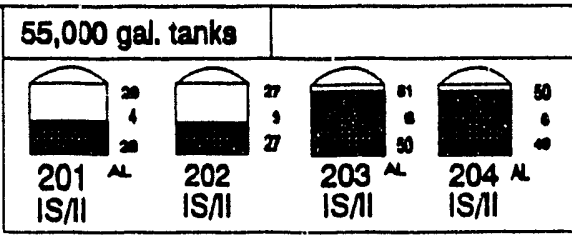

500,000 gal. tanks Constructed 1946-47

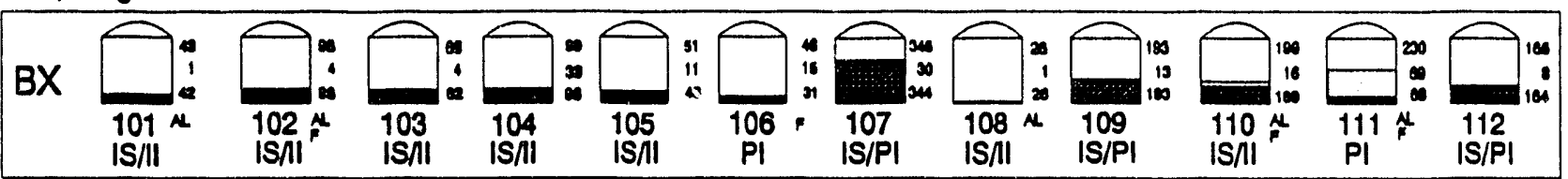

750,000 gal. tanks Constructed 1948-49

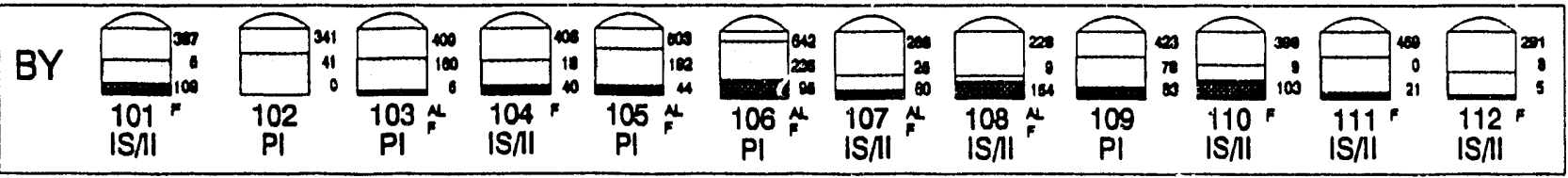

500,000 gal. tanks Constructed 1943-44

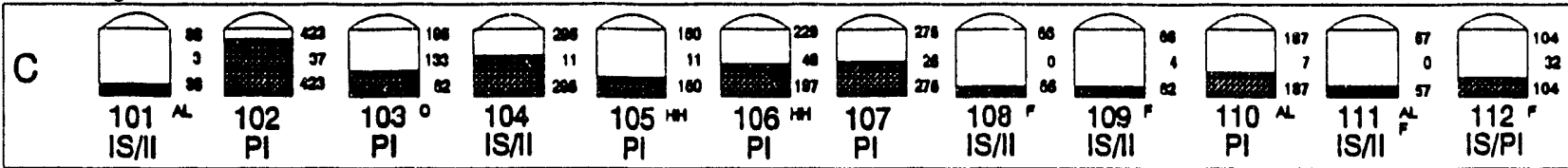

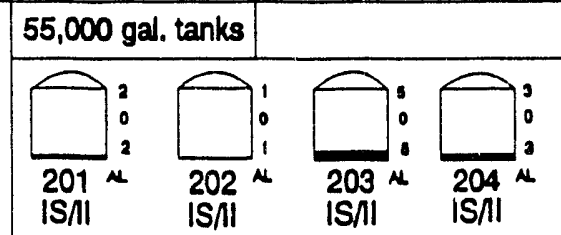

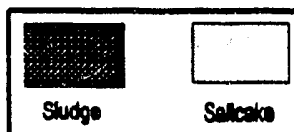

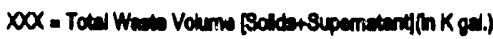

DOX $=$ Total lquids (in K gal.) Dreinabla intersiliel + Supenaton

DOX - suchos (in K geil)

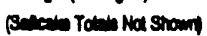

$M=$ Acoumed Laake

$F=$ Formate

micerasos

$\mathrm{O}=$ Oromics

$H=$ Potentid Finmable Gasea

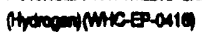

Updated Quarterly 12/31/92
$11=$ intartin isoleced

IS a intertm Stanined

$\mathrm{PI}$ a Puetialy htorm isolated

SSTAULS.C. SPENCERMO-93

Figure B-5. 200E Single-Shell Tank Status 
WHC-EP-0182-57

This page intentionally left blank.

B-8 
750,000 gal. tanks Constructed $1950-51$

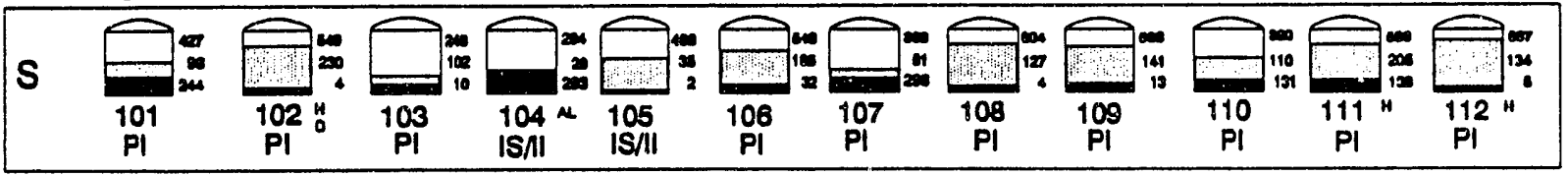

1,000,000 gal. tanks Constructed 1953-54

$S X \sum_{i=12}^{10}$

500,000 gal. tanks Constructed 1943-44

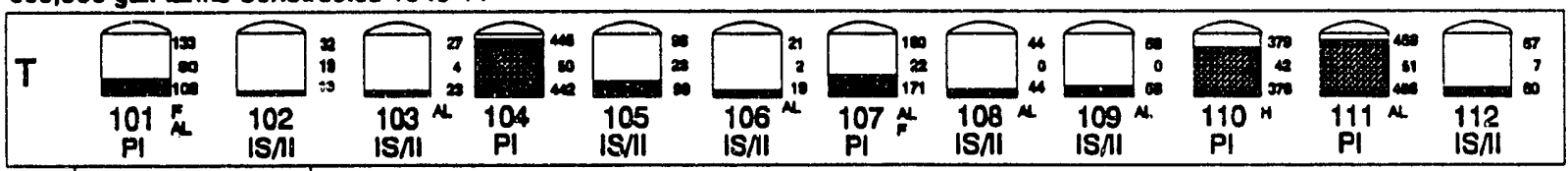

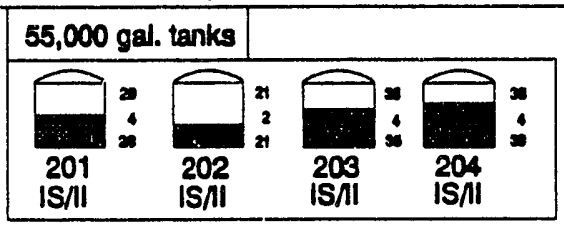

750,000 gal. tanks Constructed 1947.48

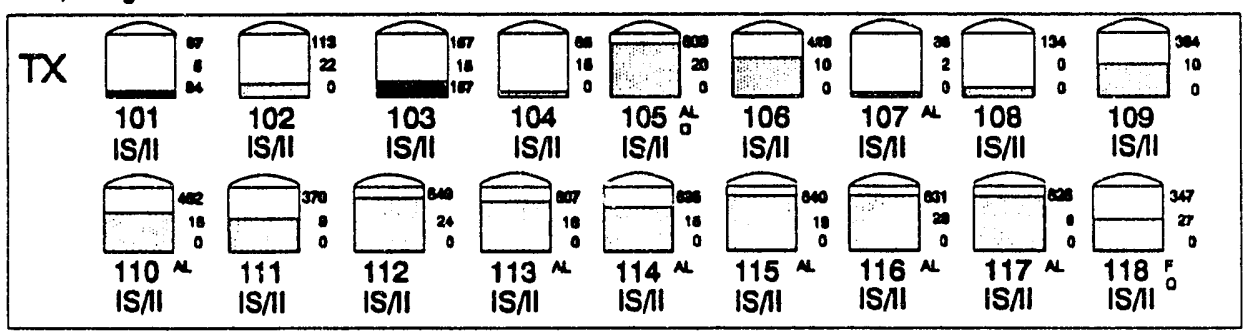

$750,000 \mathrm{gal}$, tanks Constructed 1951.52

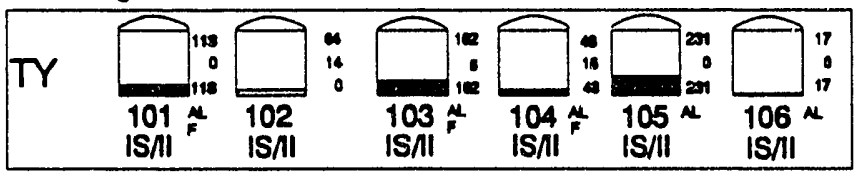

500,000 gal. tanks Constructed 1943-44

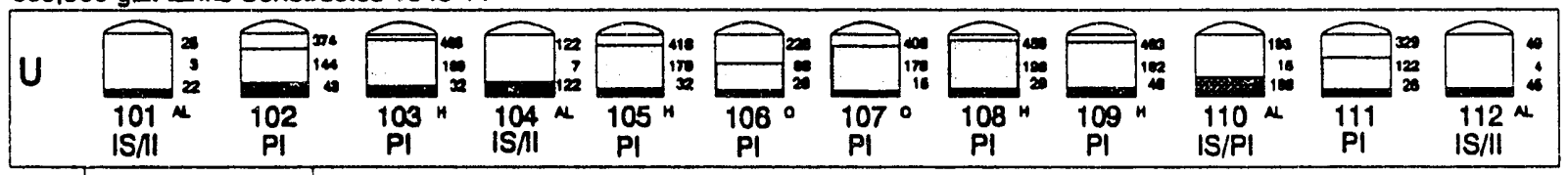

55,000 gal. tanks

\begin{tabular}{|c|c|c|c|}
\hline $\begin{array}{l}201 \\
\text { IS } 11\end{array}$ & $\begin{array}{l}202 \\
\text { IS } M 1\end{array}$ & $\begin{array}{l}203 \\
\text { IS } / 1\end{array}$ & $\begin{array}{l}204 \\
15 / 1\end{array}$ \\
\hline
\end{tabular}

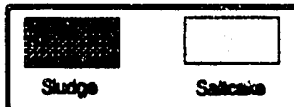

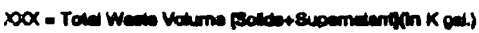
DOX - Tole haviles (in K oed)

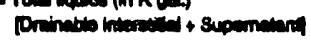

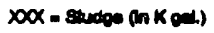

Tom
N - A Neamed Lenter

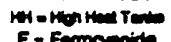

$F$ a Ferrocyanids

O- Oranice

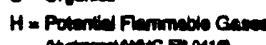
androminuic-erosia

Updated Quarterly 12/31/92
H. Irivenm bolened

IS - Interm Steblized

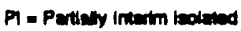

SST-NUS.G. SPENCEROO-P

Fig. B-6. 200W Singie-Sheil Tank Status 
WHC-EP-0182-57

This page intentionally left blank. 


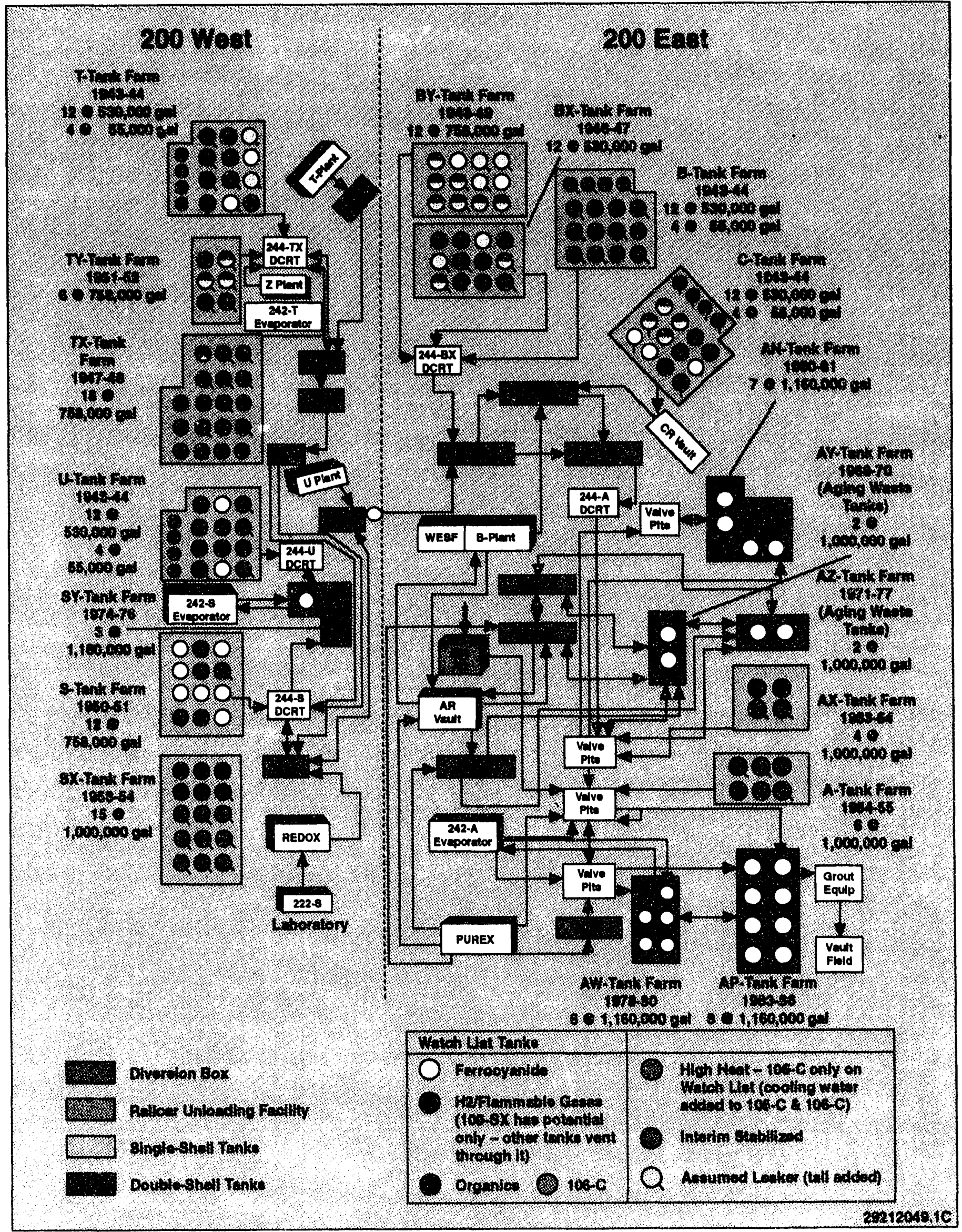

Figure B-7. Tank Farm Facilities - Quick Reference 


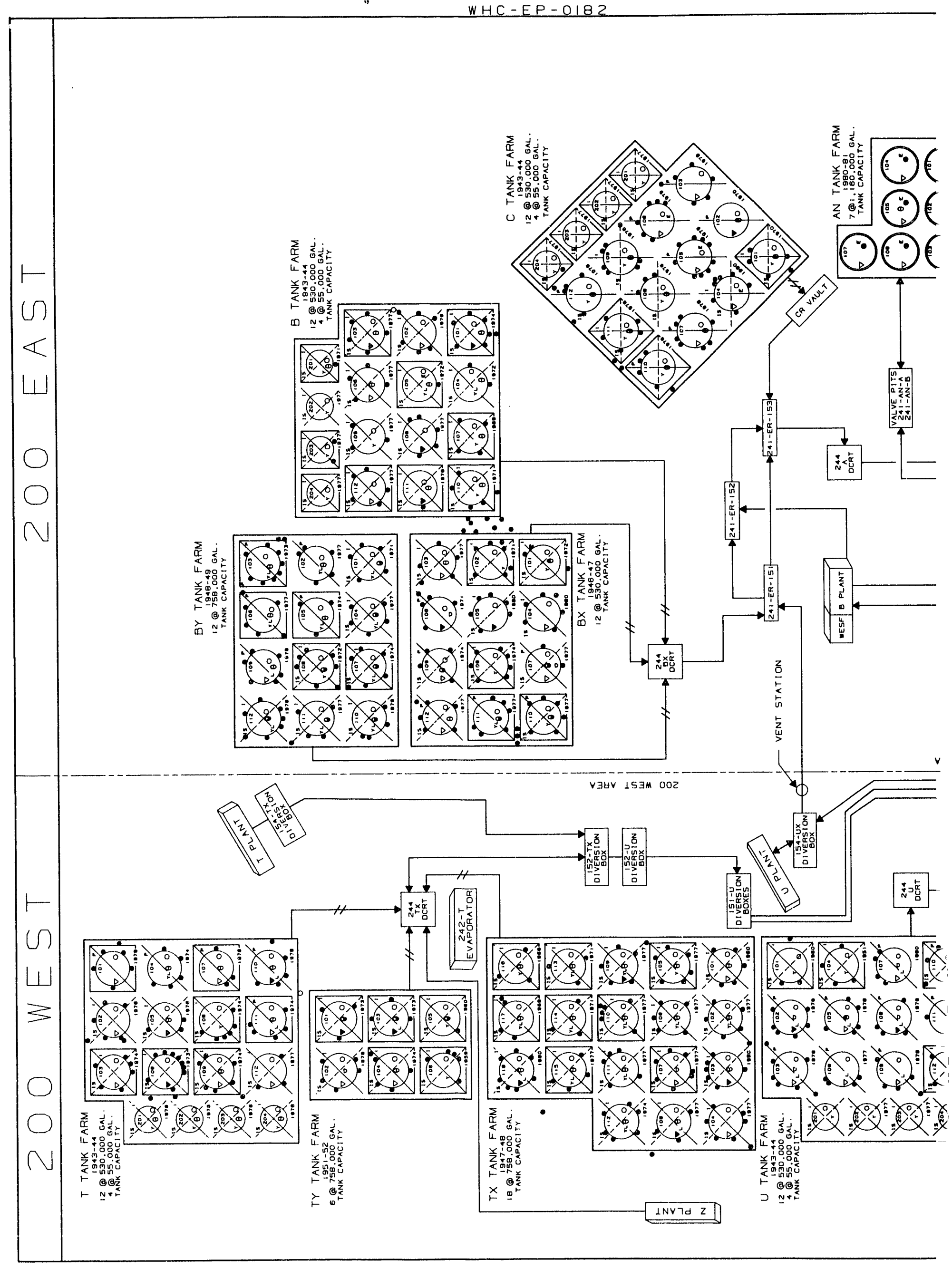




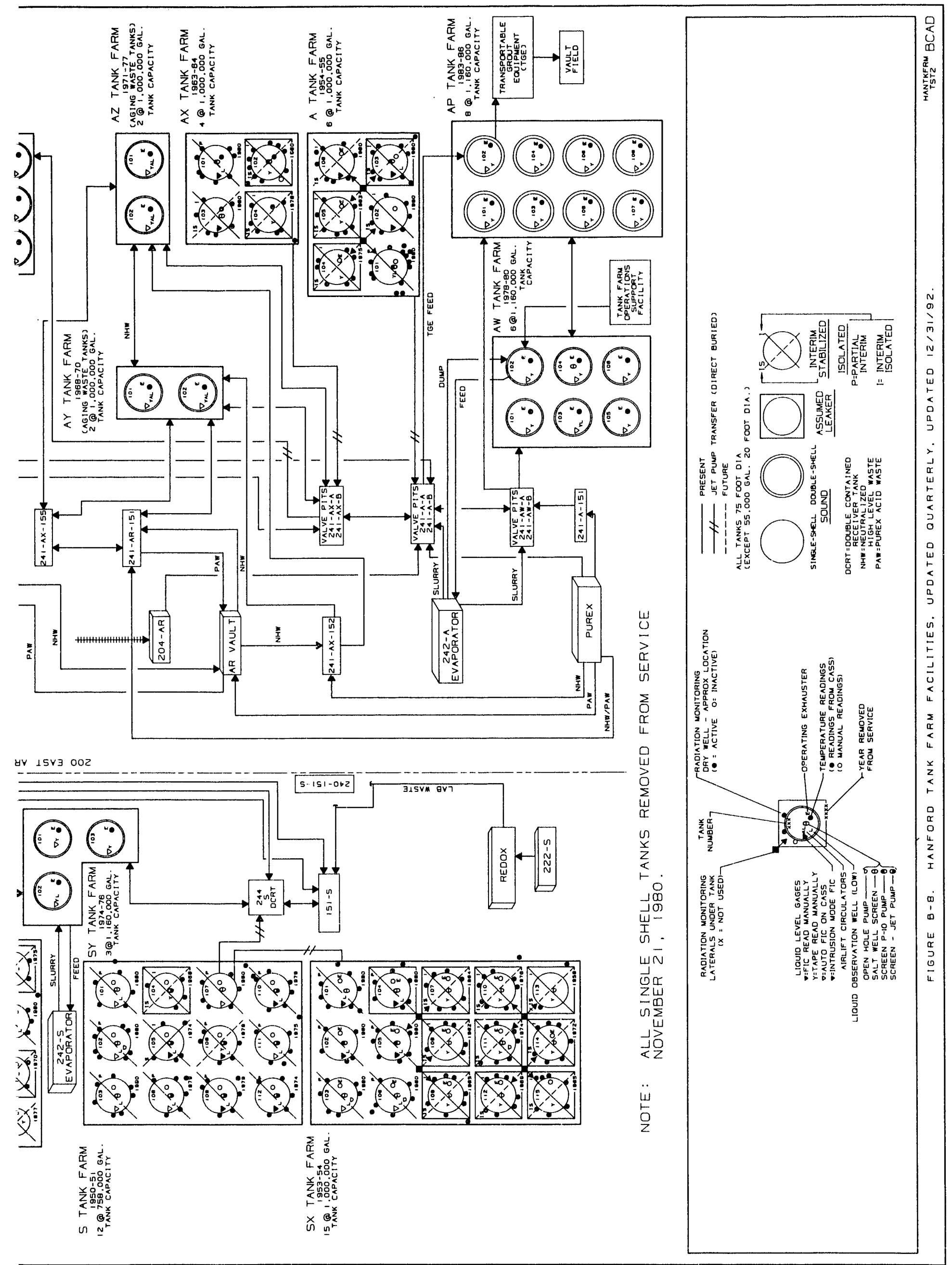


WHC-EP-0182-57

\section{APPENDIX C}

MONTHLY SUMMARY

TANK USE SUMMARY

INVENTORY SUMMARY BY TANK FARM INVENTORY AND STATUS BY TANK 
WHC-EP-0182-57

This page intentionally left blank. 


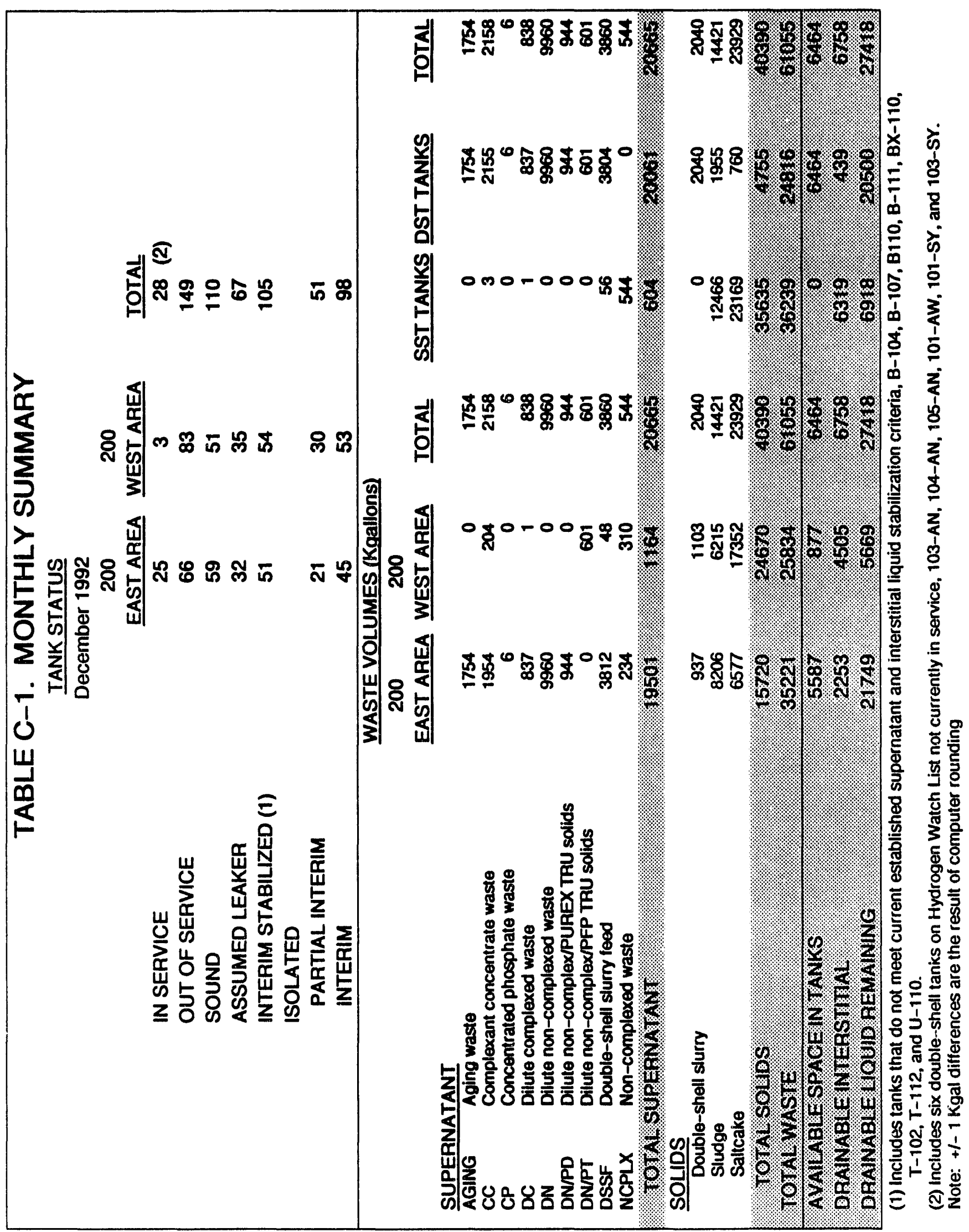


WHC-EP-0182-57

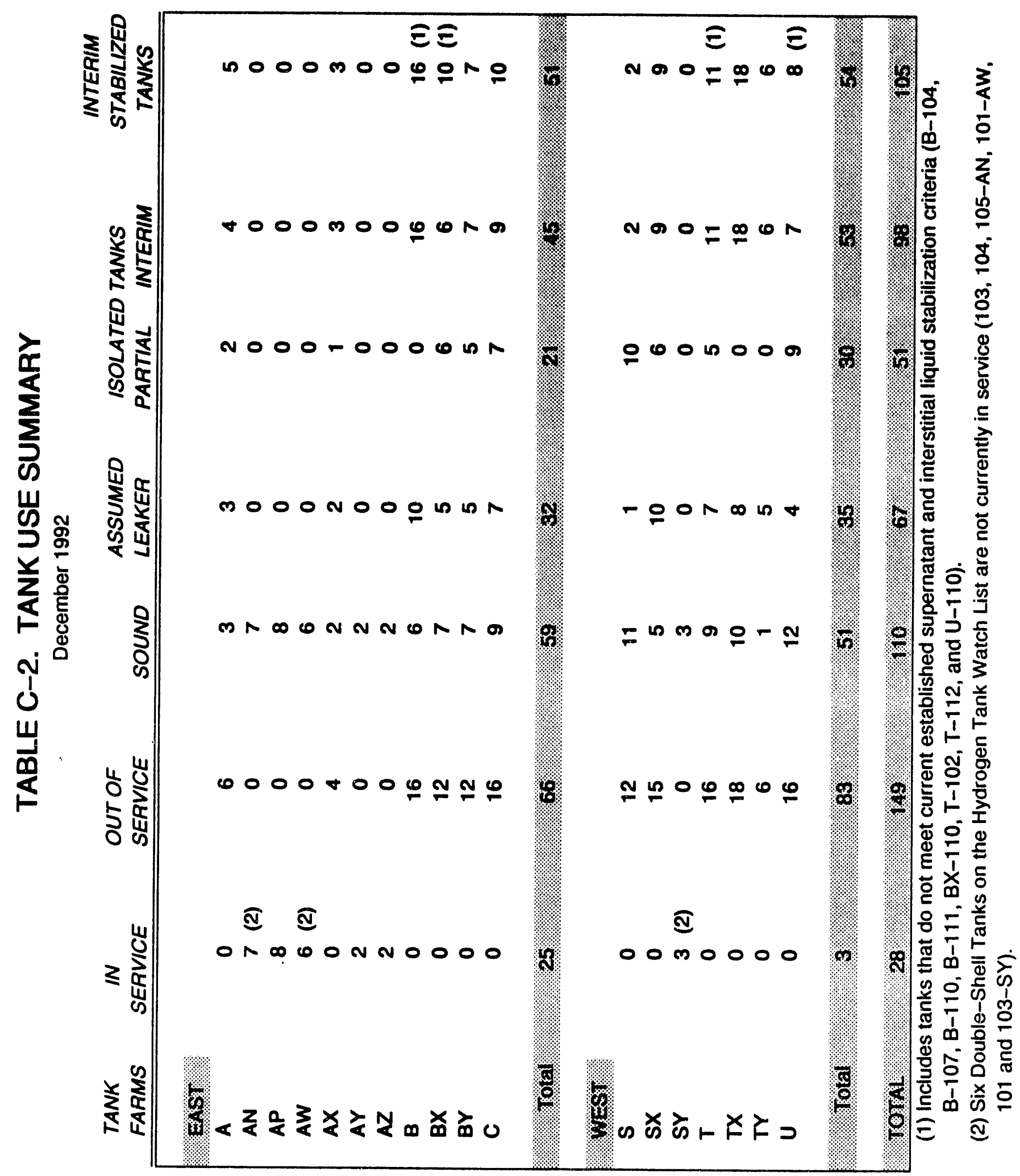




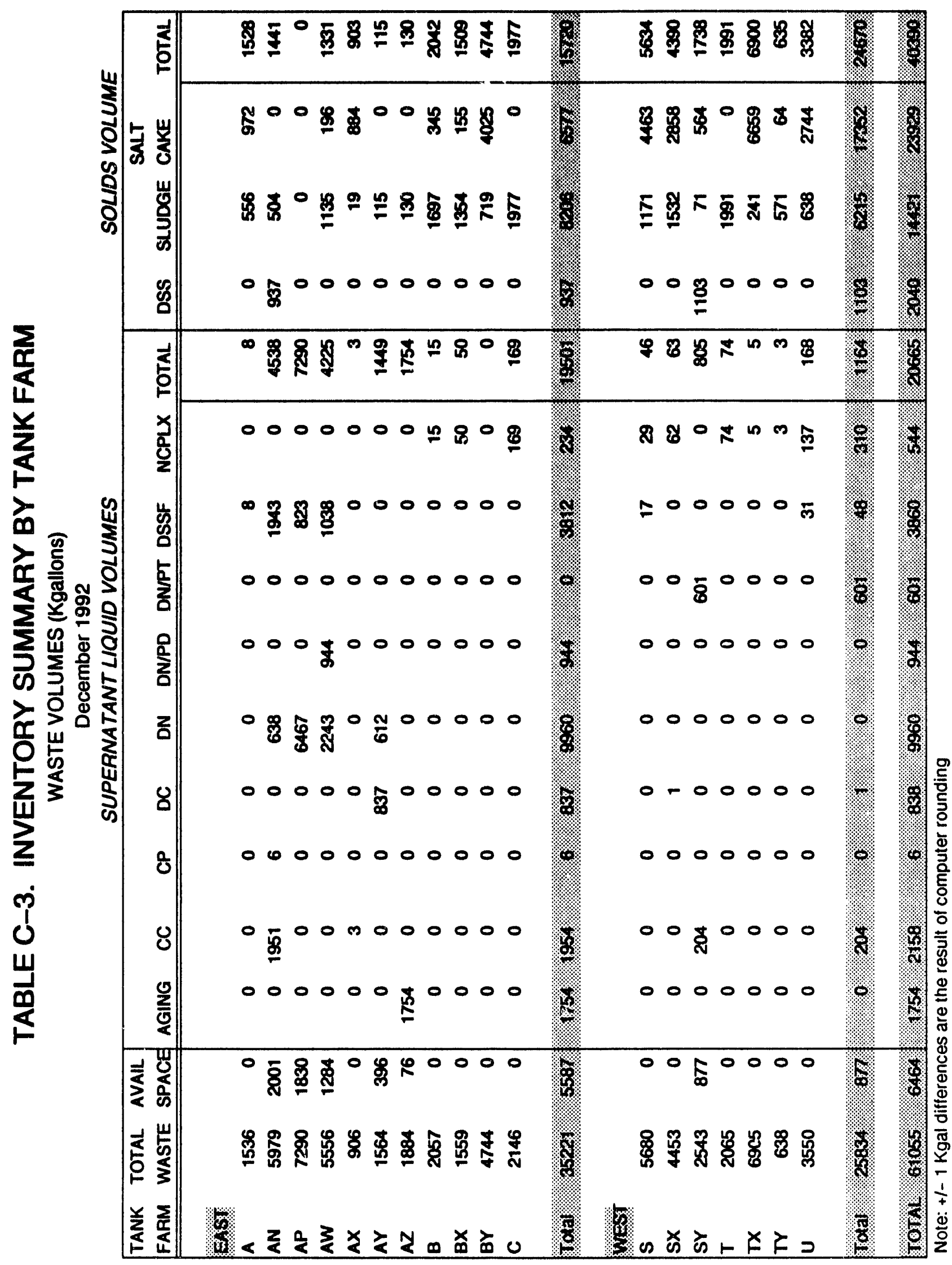




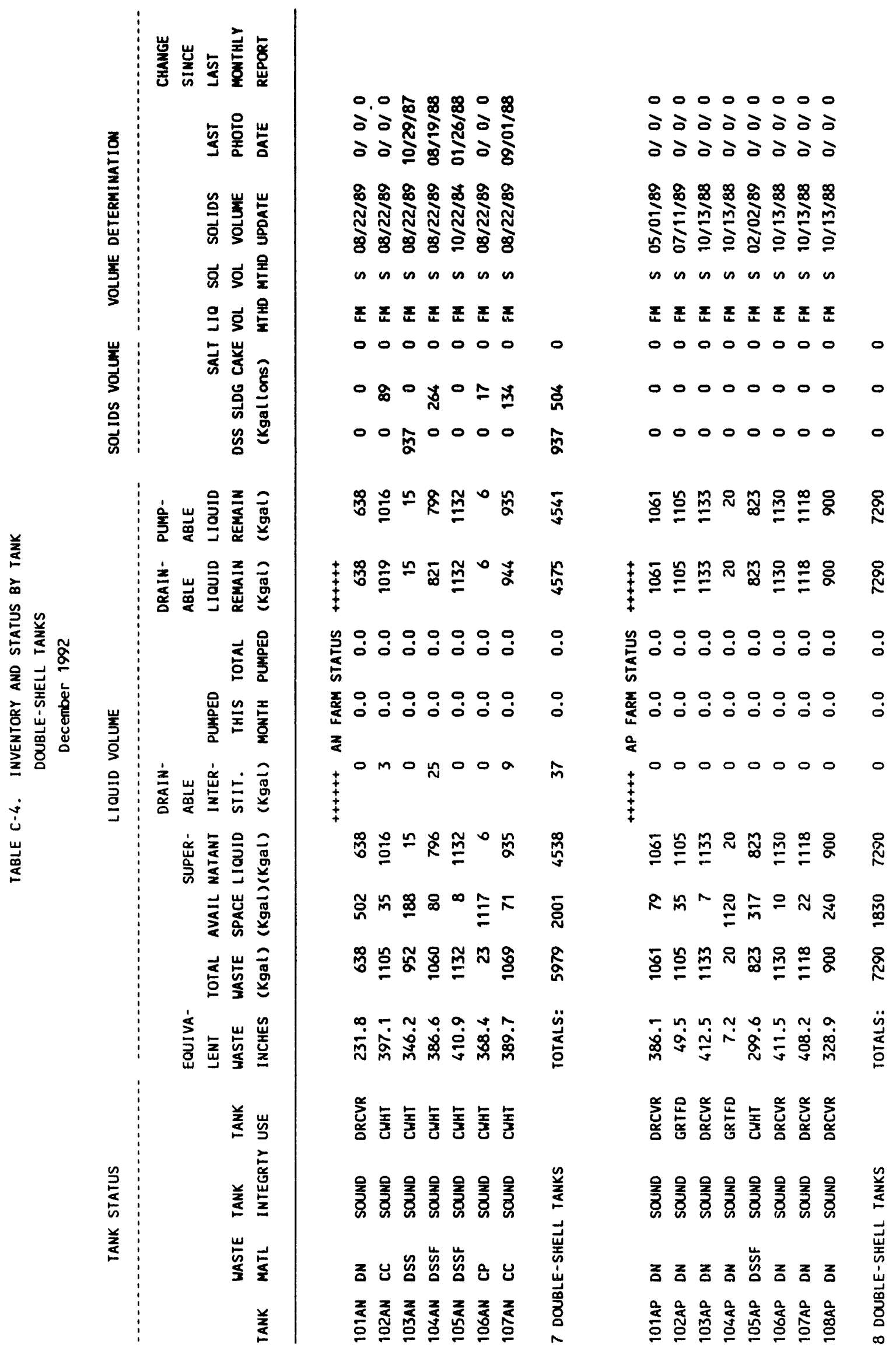




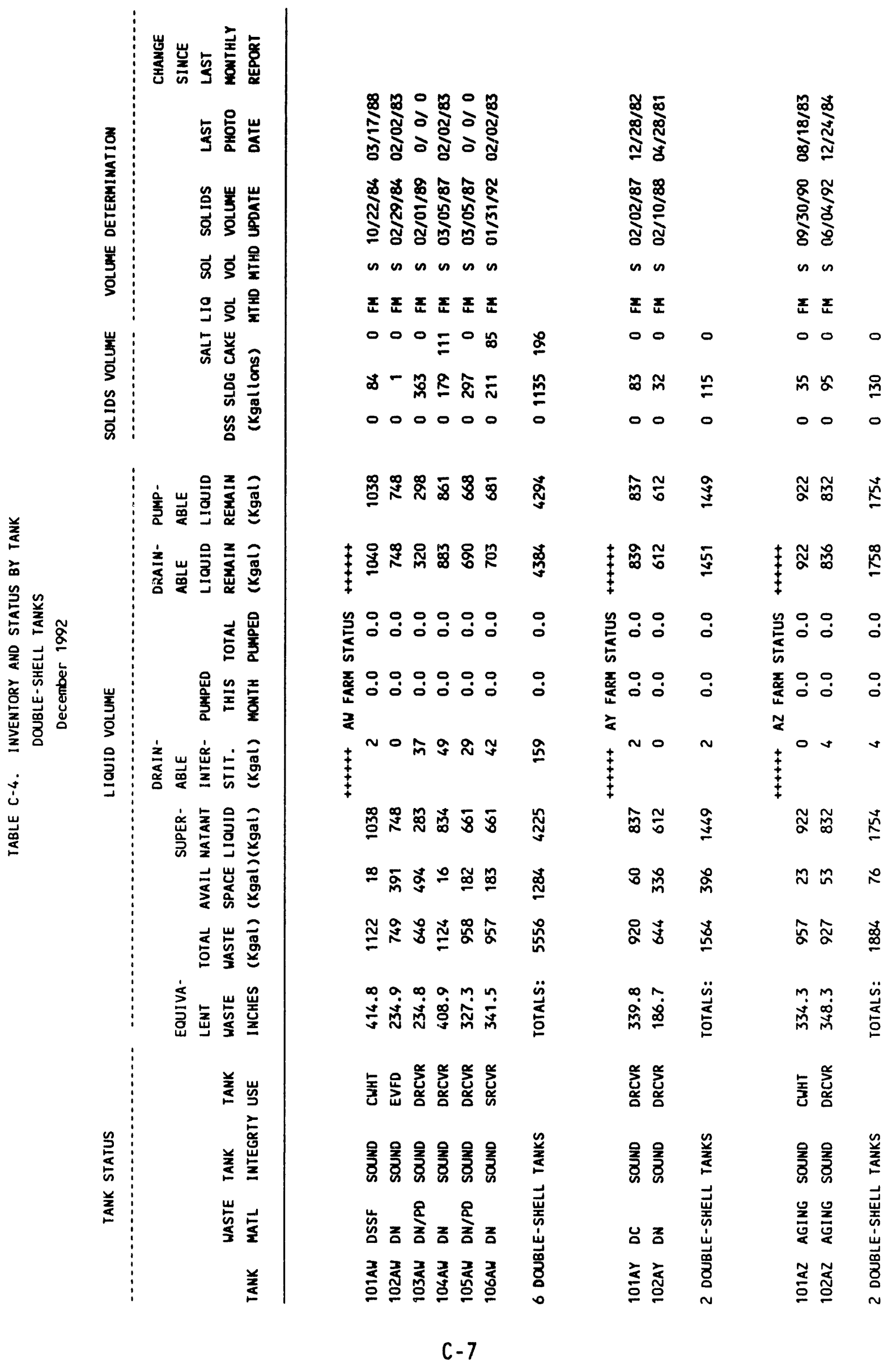




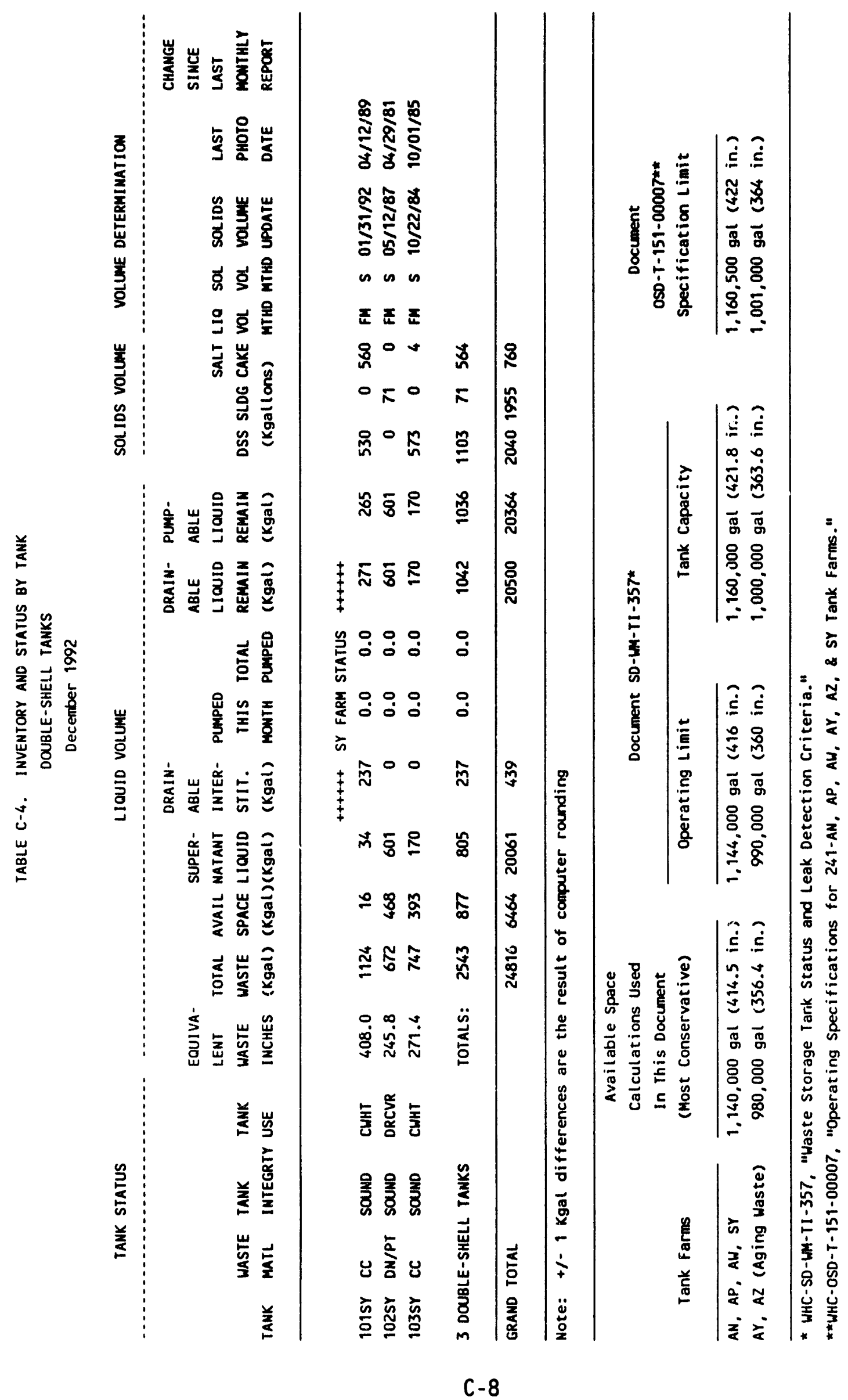




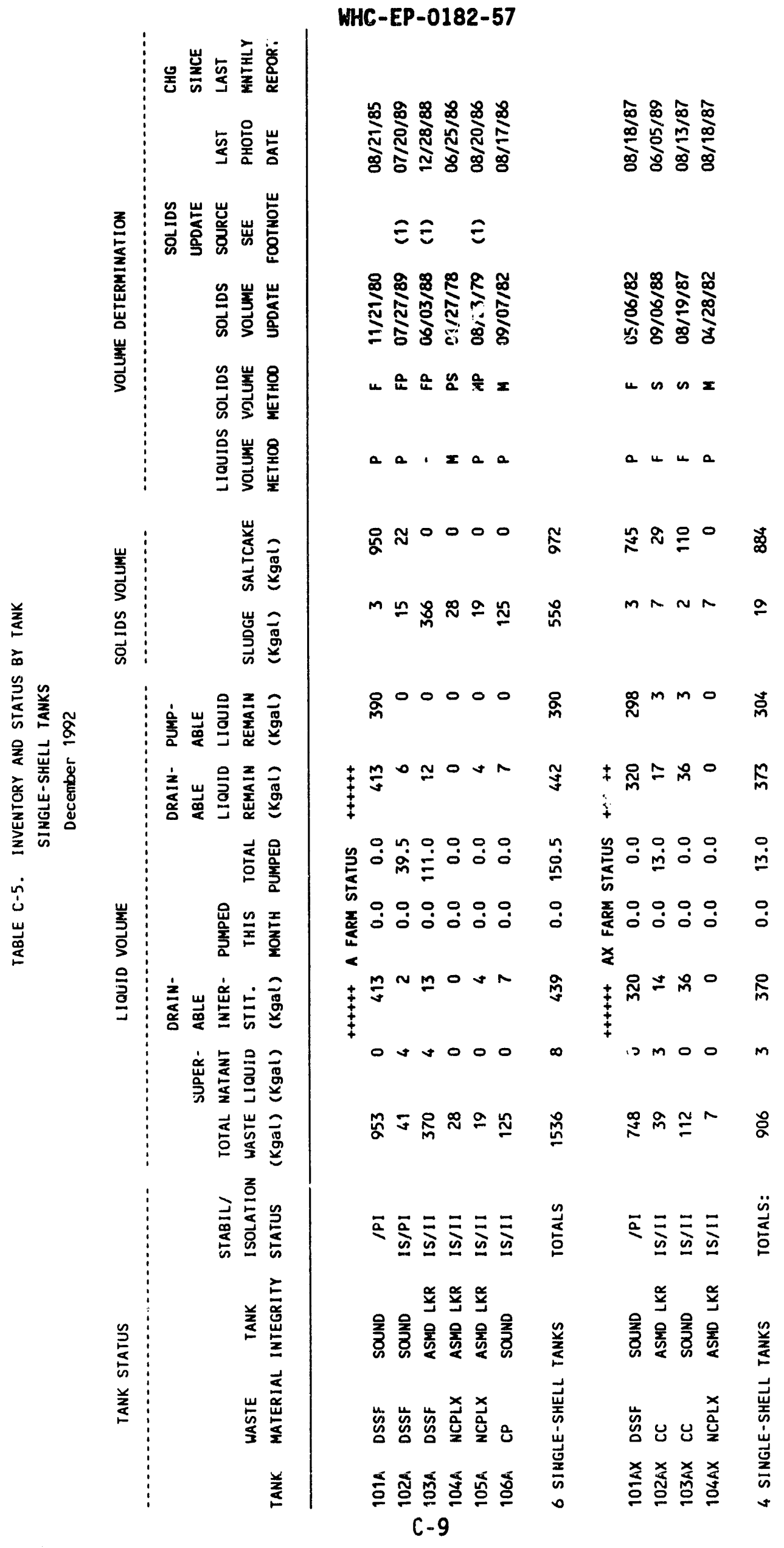




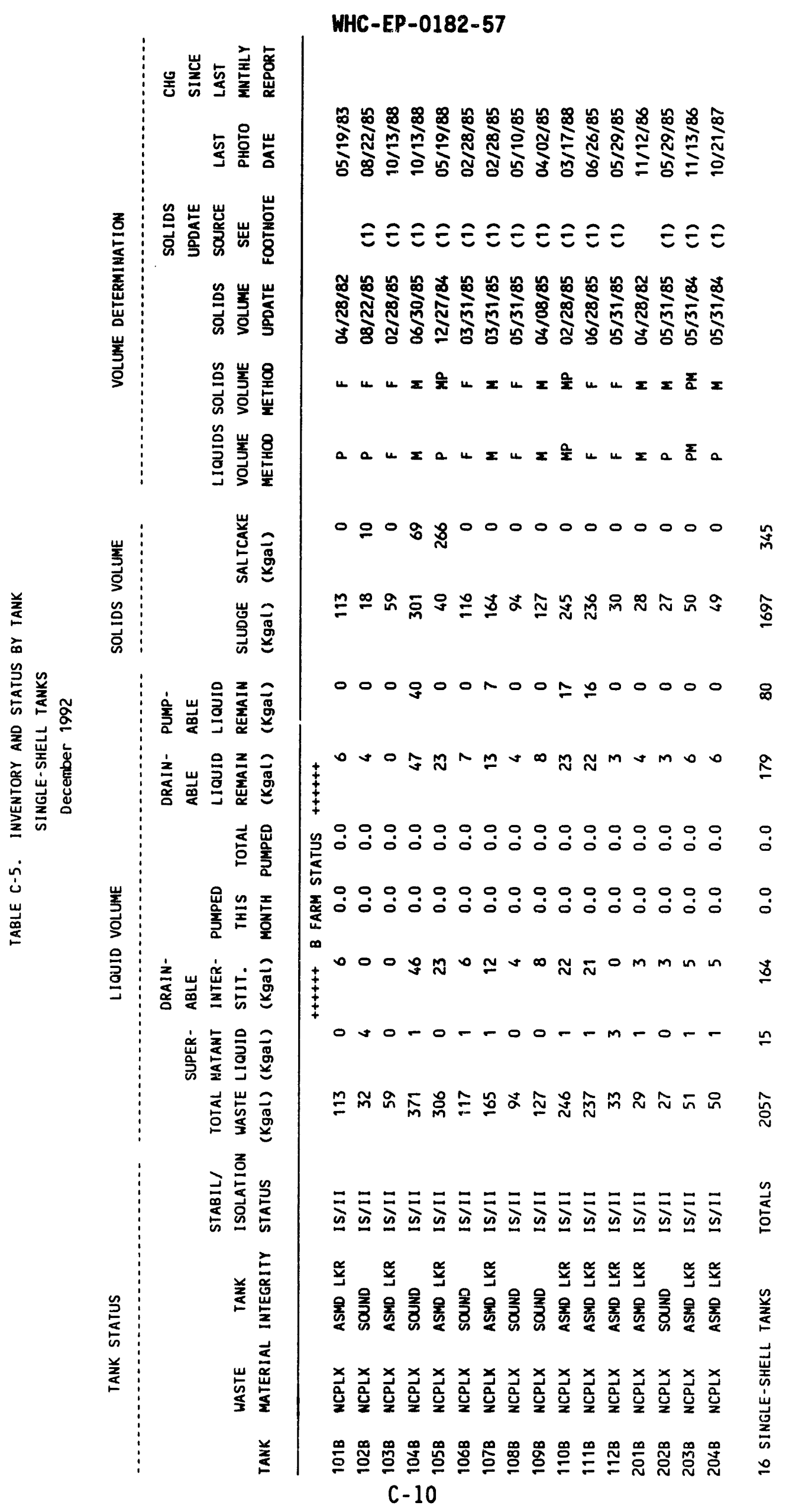




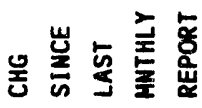

毞 움 음

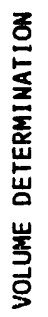

(

岂岕岕岕㟯

崖崖崖

崖 8

熍

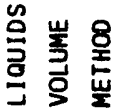

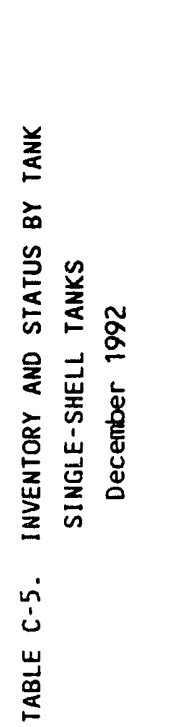

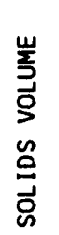

峞

岁 蛋

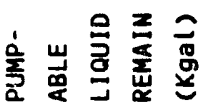

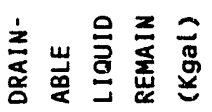

运 鵕

㸚

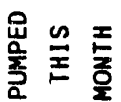

WHC-EP-0182-57

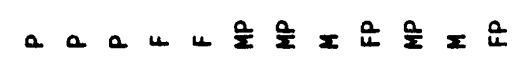

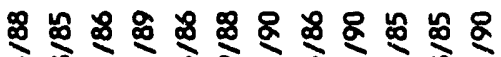

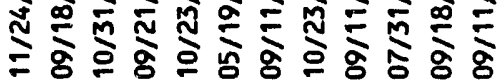

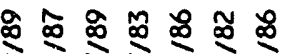

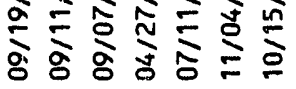

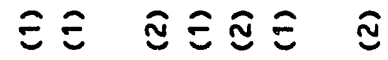

ลิ ลิ

๘

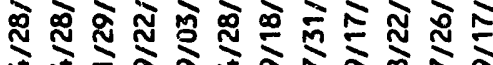

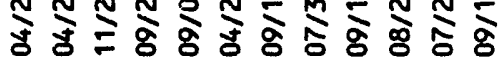

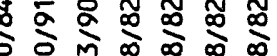

mi mid

X $x$ แ แ 以

$\Sigma \Sigma \Sigma \sum \frac{0}{2} \frac{0}{2} \frac{0}{2}$

a $\frac{a}{2} \frac{a}{2} a a^{2} a$

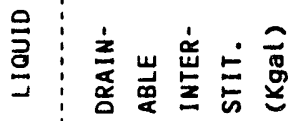

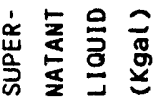

志崖票

$0000 m 00000 \frac{2}{3}$

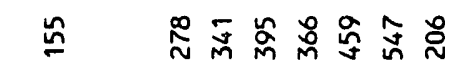

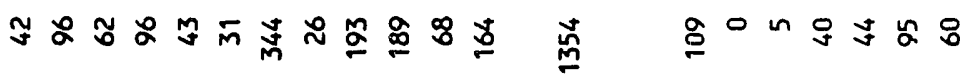

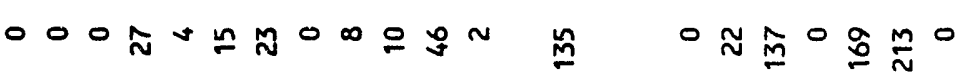

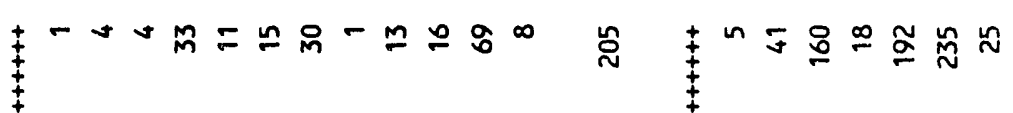

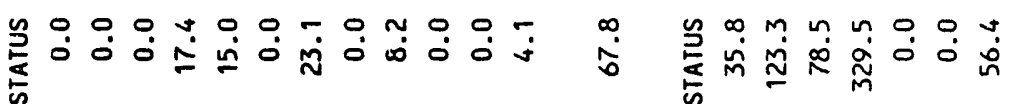

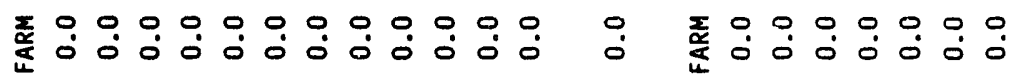
希

tor

㐫 $\stackrel{+}{+}$

- ovm nn $n$ - o o- - -

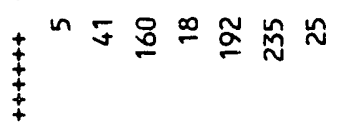

0000000

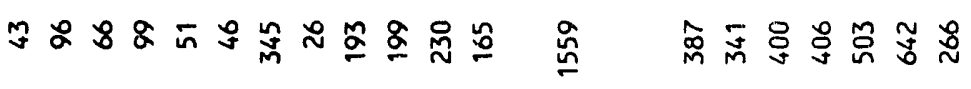

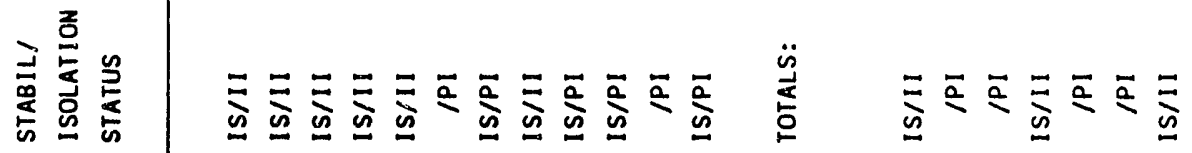

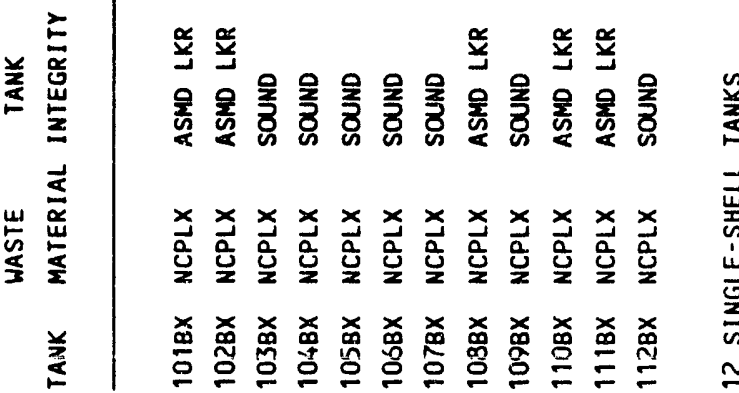

C-11 


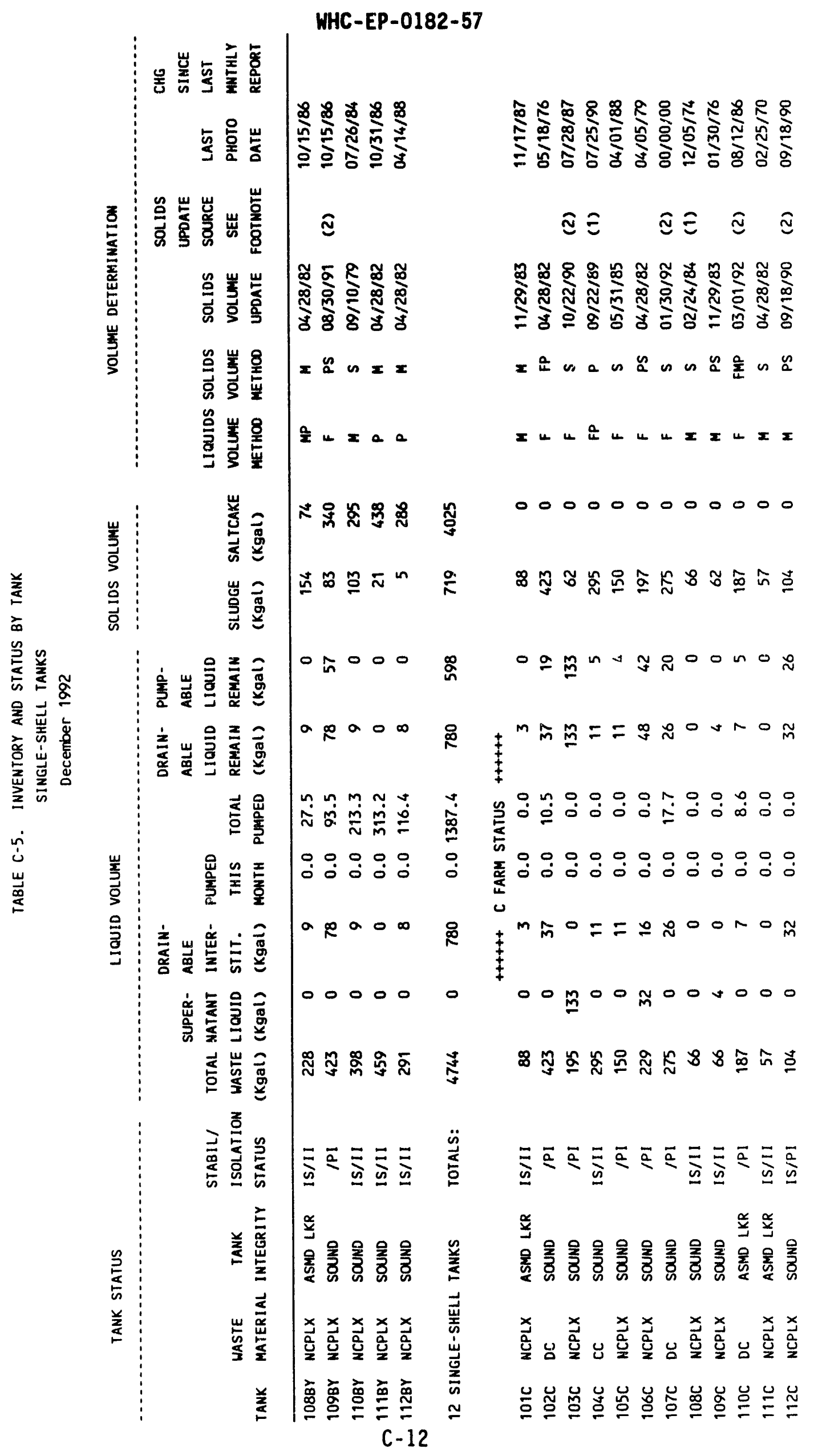




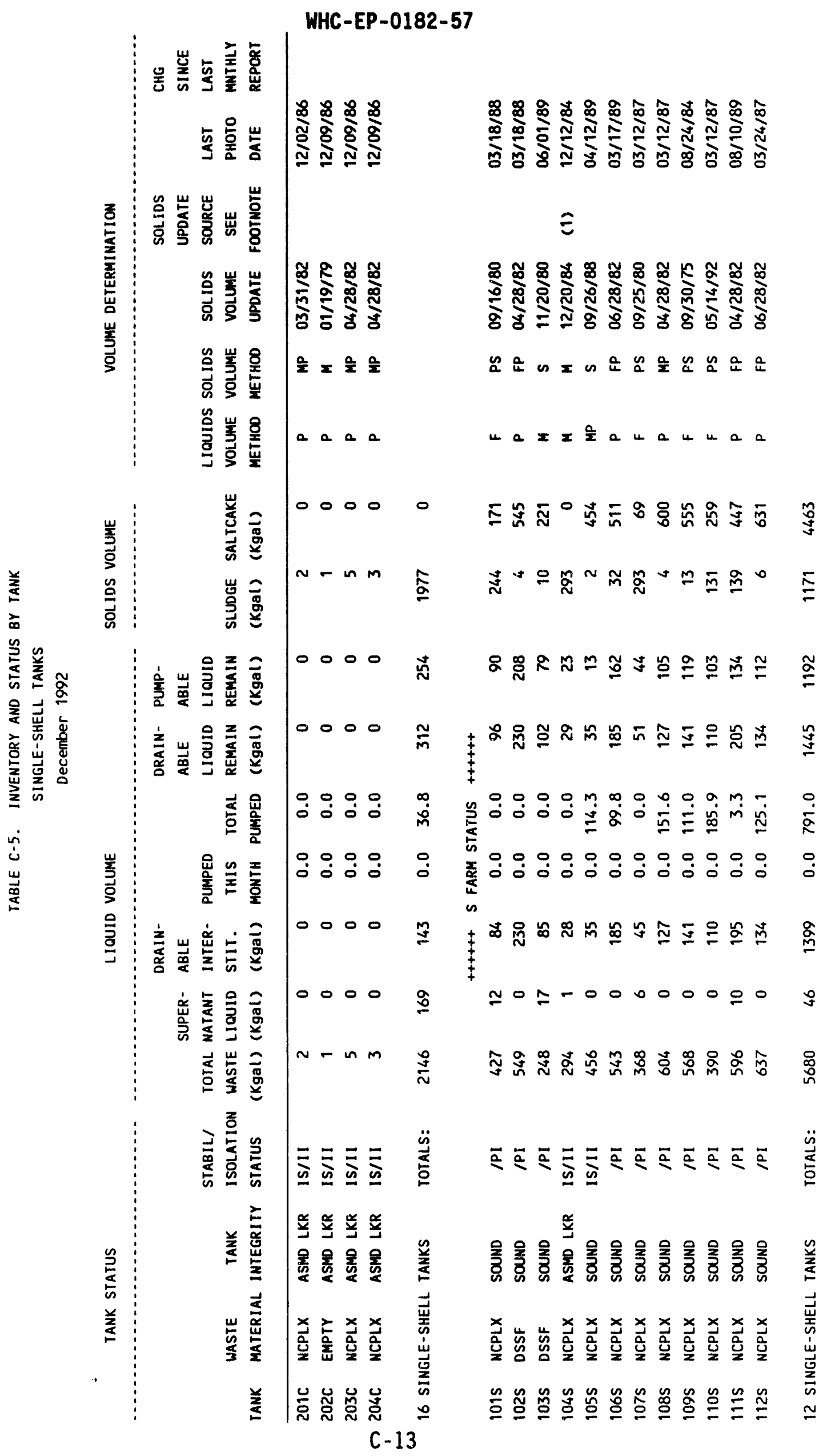




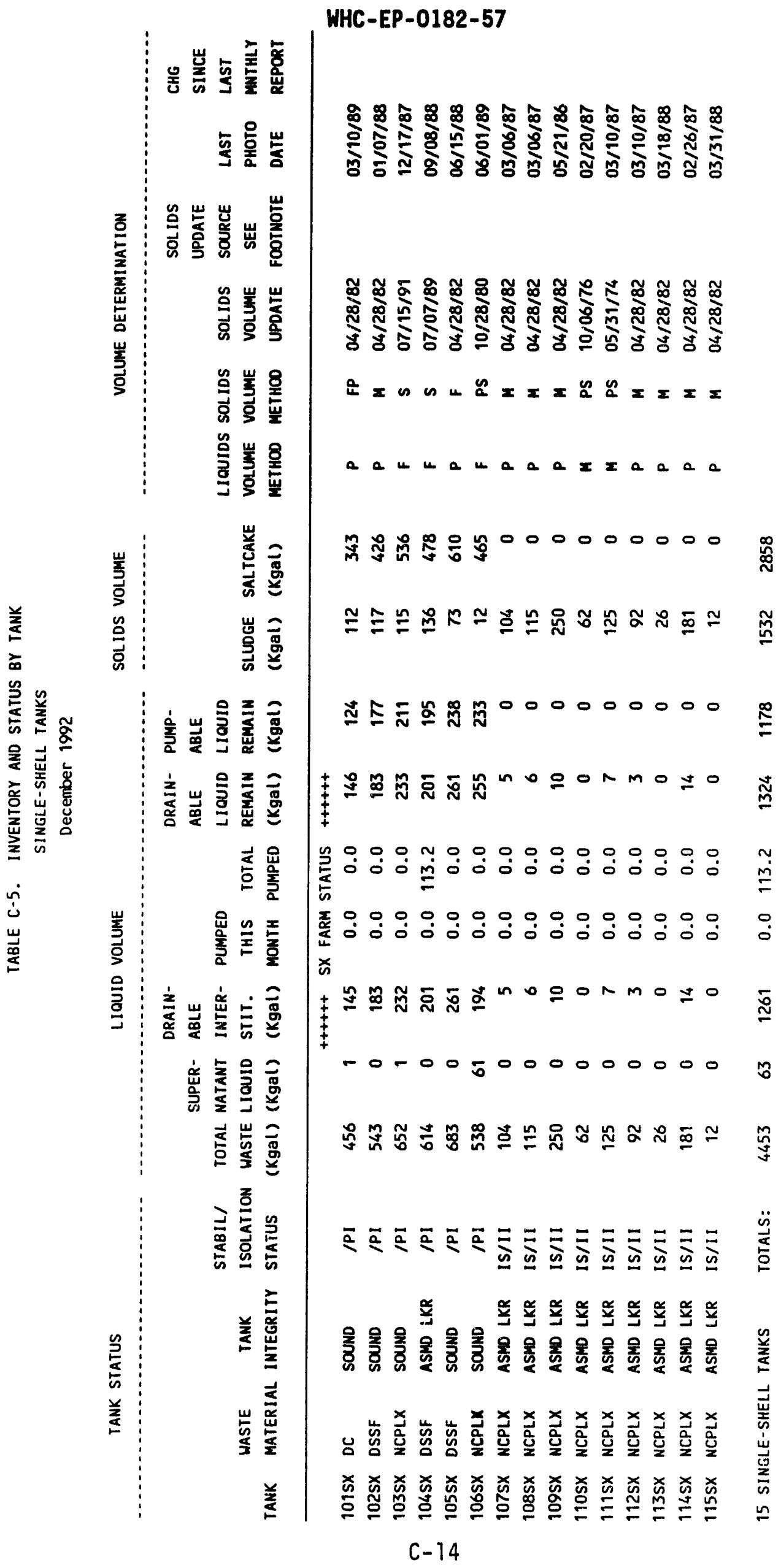




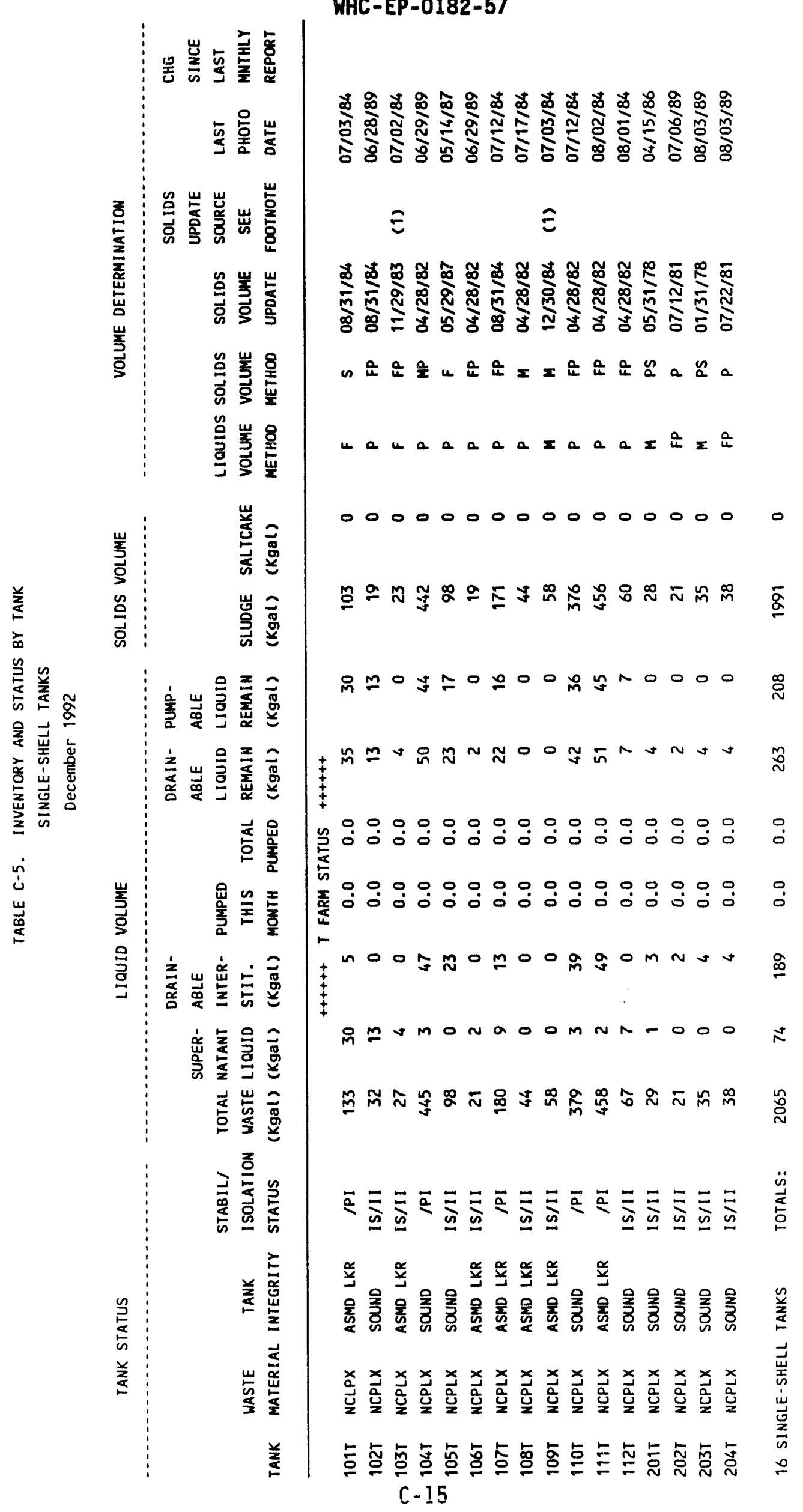




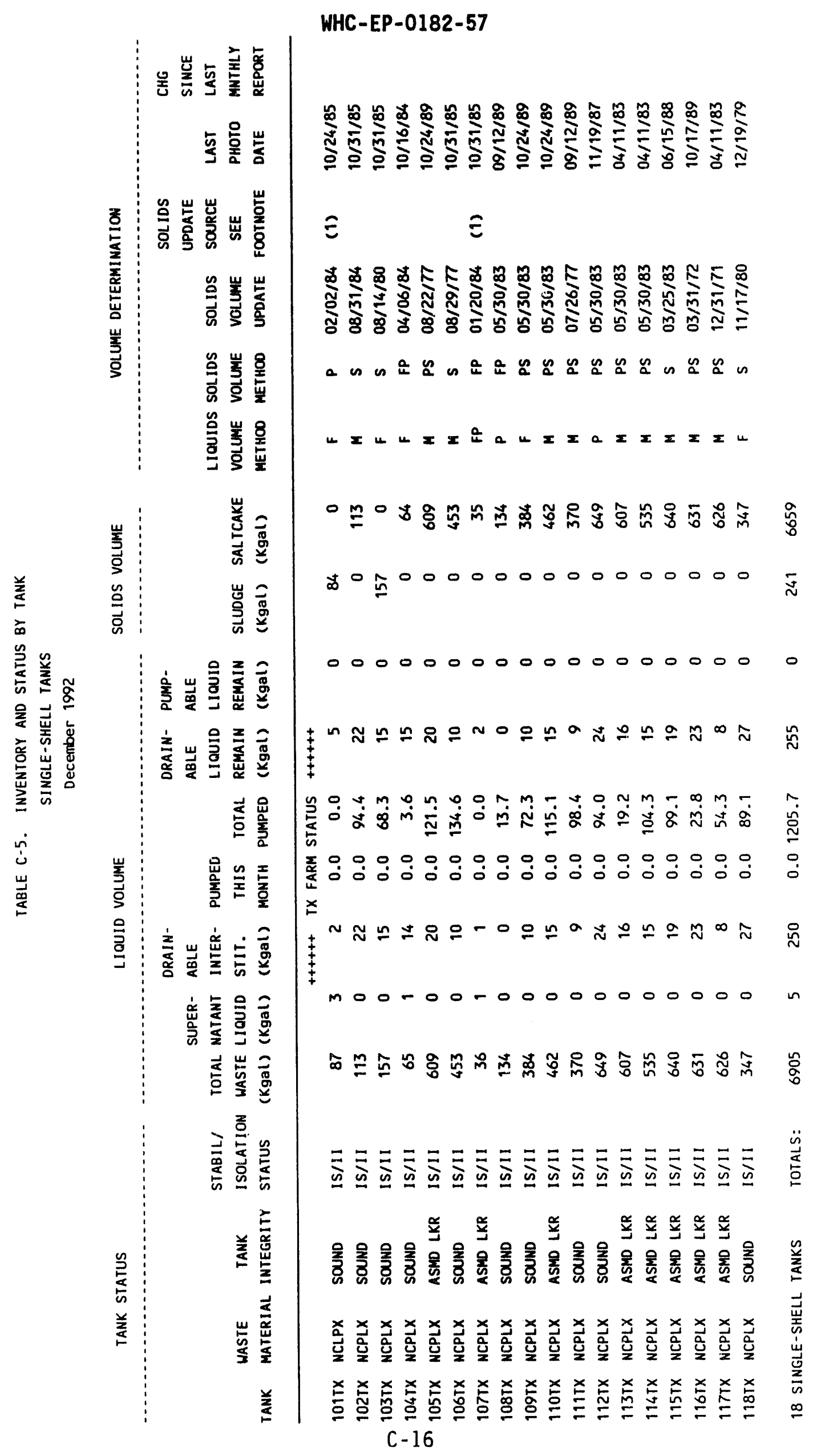




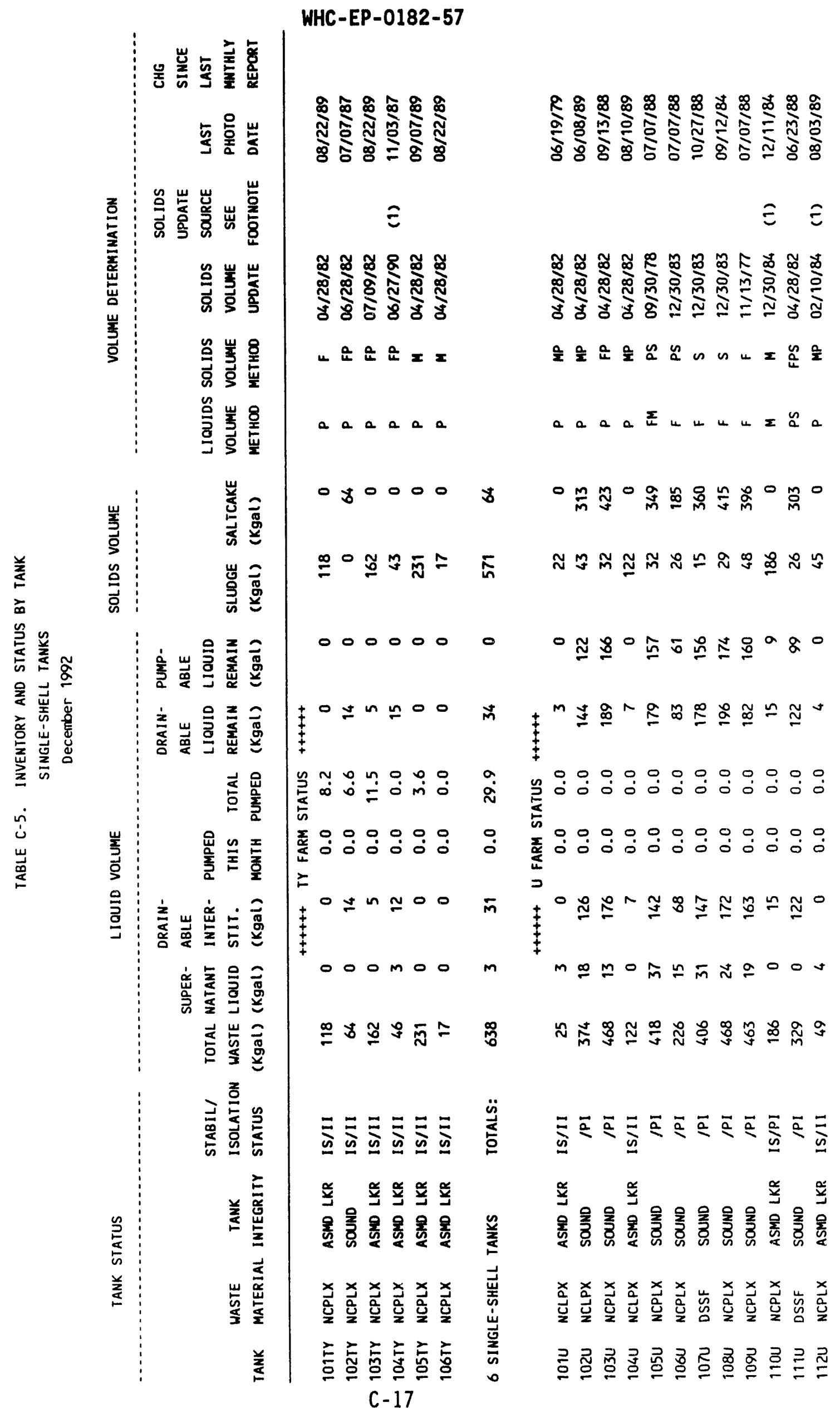




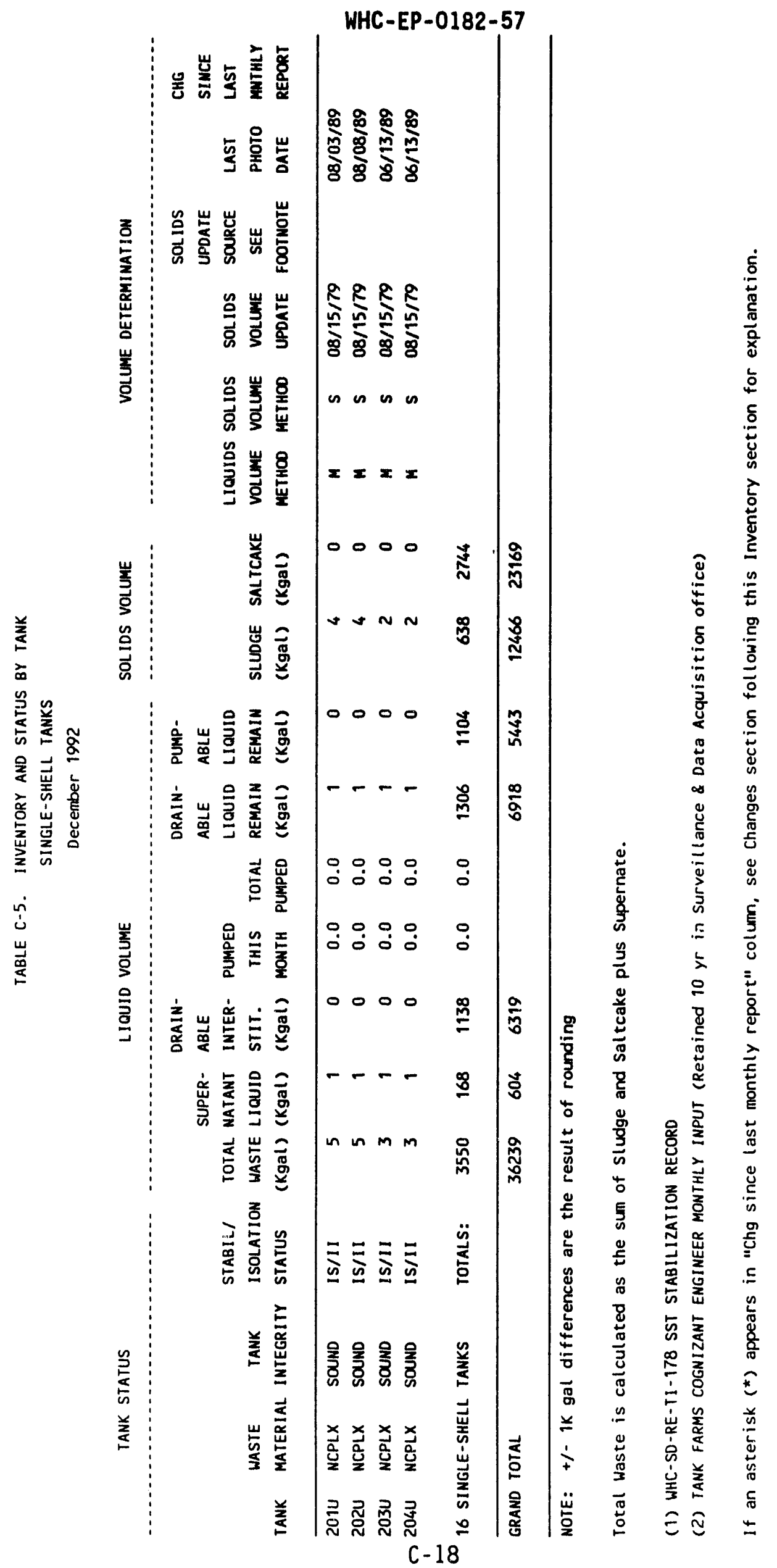


WHC-EP-0182-57

APPENDIX D

PERFORMANCE SUMMARY

D-1 
WHC-EP-0182-57

This page intentionally left blank. 


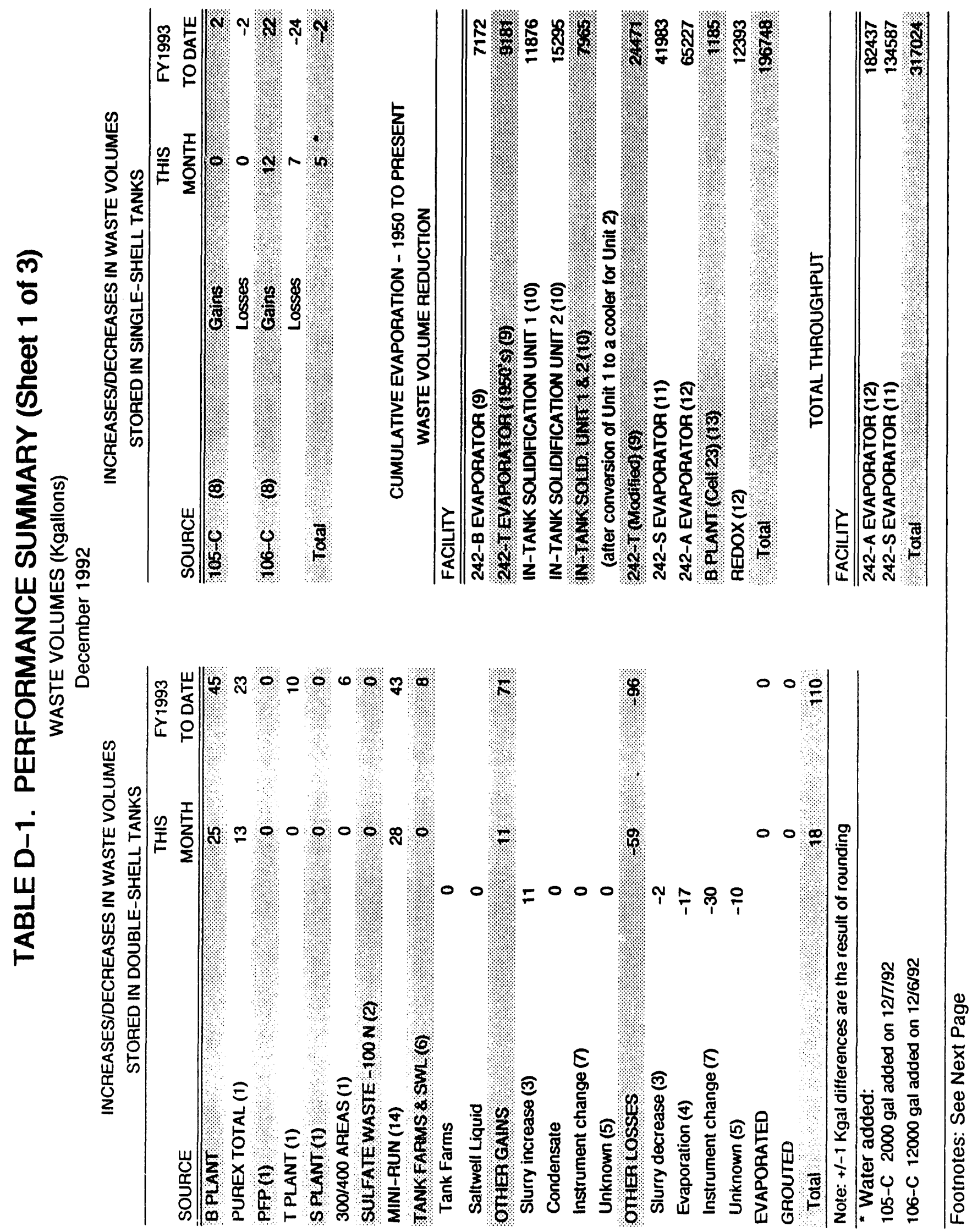


TABLE D-1. Performance Summary (Sheet 2 of 3 )

Footnotes:

\section{INCREASES/DECREASES IN WASTE VQLUMES}

\section{(1) Including Flush}

(2) Sulfate waste is generated from ion exchange backflushing and sand filter clean out, resulting in sulfate waste $\left(\mathrm{Na}_{2} \mathrm{SO}_{4}\right)$.

(3) Slurry increase/growth is caused by gas generation within the waste. The gas which is trapped in the waste expands in the tank causing the surface level and volume to increase. Slurry decrease results from the periodic release of gas in the waste.

(4) Aging waste tanks

(5) Unknown waste gains or losses may be the result of rounding calculations, clean water slowly leaking through a valve, changes in levels (expansion/constraction) because of to ambient temperature changes, different measuring devices being used by Tank Farm operators, transfers taking place during the end of the month, Tank Farm activities such as miscellaneous water additions not associated with facility waste generation, or the addition of water which is added to aging waste tanks and then evaporated off.

(6) Includes Tank Farms miscellaneous flushes (flushes are used to "clean out" pipelines and reduce personnel exposure, reduce potential for waste incompatibility, prevent line plugging, and reduce waste content of potential spills or leaks), and saltwell liquid, which results from pumping of single-shell tanks to double-shell tanks.

(7) Liquid level measurement instrument changes from the automatic FIC to manual tape (and vice versa) result in unusual gains or losses because the manual tape may rest on an uneven crust surface giving a different reading from that of the automatic FIC. These instrument changes are made when the automatic FIC is out of service and the reading from the manual tape is used for reporting purposes. The reported reading reverts back to the automatic FIC when it is repaired.

(8) Water is periodically added to 105-C and 106-C to provide evaporative cooling. Losses due to evaporation are calculated assuming al1 losses are evaporative losses. Drywell monitoring for leak detection is done monthly on tank 105-C. Some drywells are monitored weekly and some are monitored every two weeks on tank 106-C. If there are any indications of a leak from these tanks, the assumption that all losses are due to evaporation will be reevaluated. 


\section{WASTE VOLUME REDUCTION}

(9) Currently inoperative. These evaporator systems (242-B and 242-T) were installed in 1952 in each of the two operating areas to remove water from the waste, and ran for approximately 4 yr after which both units were shut down. The 242-T Evaporator was reactivated in December 1965, and shut down again in April 1976.

(10) Currently inoperative. These two in-tank solidification (ITS) units provided in-tank heating to promote in-tank boiling or evaporation. The ITS Unit l started up March 1965, and ITS Unit 2 started up February 1968. In August 1971, ITS Unit 1 was converted from an evaporator to a cooler for ITS Unit 2. Both units were shut down June 1974.

(11) Currently inoperative. The 242-S Evaporator-Crystallizer was started up November 1973, and shut down March 1980, when its processing campaign was completed. It is in standby mode with no future mission. This evaporator operates under a vacuum, employing evaporative concentration with subsequent crystallization and precipitation of salt crystals.

(12) Currently inoperative. The 242-A Evaporator-Crystallizer was started up March 1977, and shut down Apri1 1989 because of regulatory issues, and has remained shut down for subsequent upgrading. The restart schedule specifies January 4, 1993, as the projected start-up date. This evaporator operates under a vacuum, employing evaporative concentration with subsequent crystallization and precipitation of salt crystals (forming saltcake).

(13) Currently inoperative. Additional concentration of wastes was completed by using the concentrators at REDOX and B Plant. The REDOX concentrator was used from July 1967 to June 1972, while the B Plant concentrator was used from July 1967 to February 1968.

(14) Waste generated for training and testing purposes prior to Evaporator restart 


\section{WHC-EP-0182-57}

This page intentionaliy left blank.

D-6 
WHC-EP-0182-57

\section{APPENDIX E}

\section{LIQUID STATUS AND PUMPABLE LIQUID}

REMAINING IN TANKS 
WHC-EP-0182-57

This page intentionally left blank. 
WHC-EP-0182-57

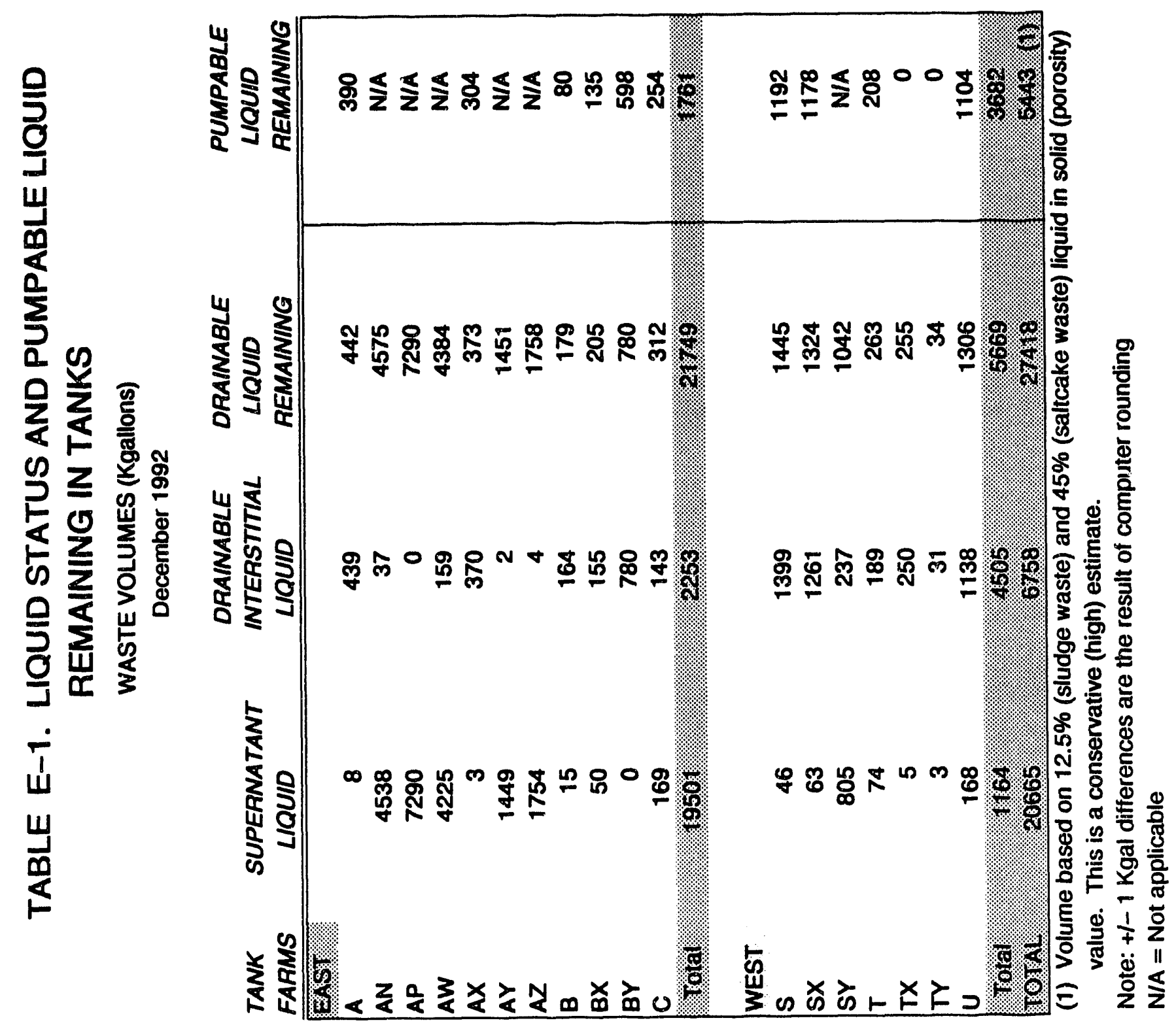


WHC-EP-0182-57

This page intentionalyy left blank.

E-4 
WHC-EP-0182-57

\section{APPENDIX F \\ PUMPING RECORD}

F-1 
WHC-EP-0182-57

This page intentionally left blank. 

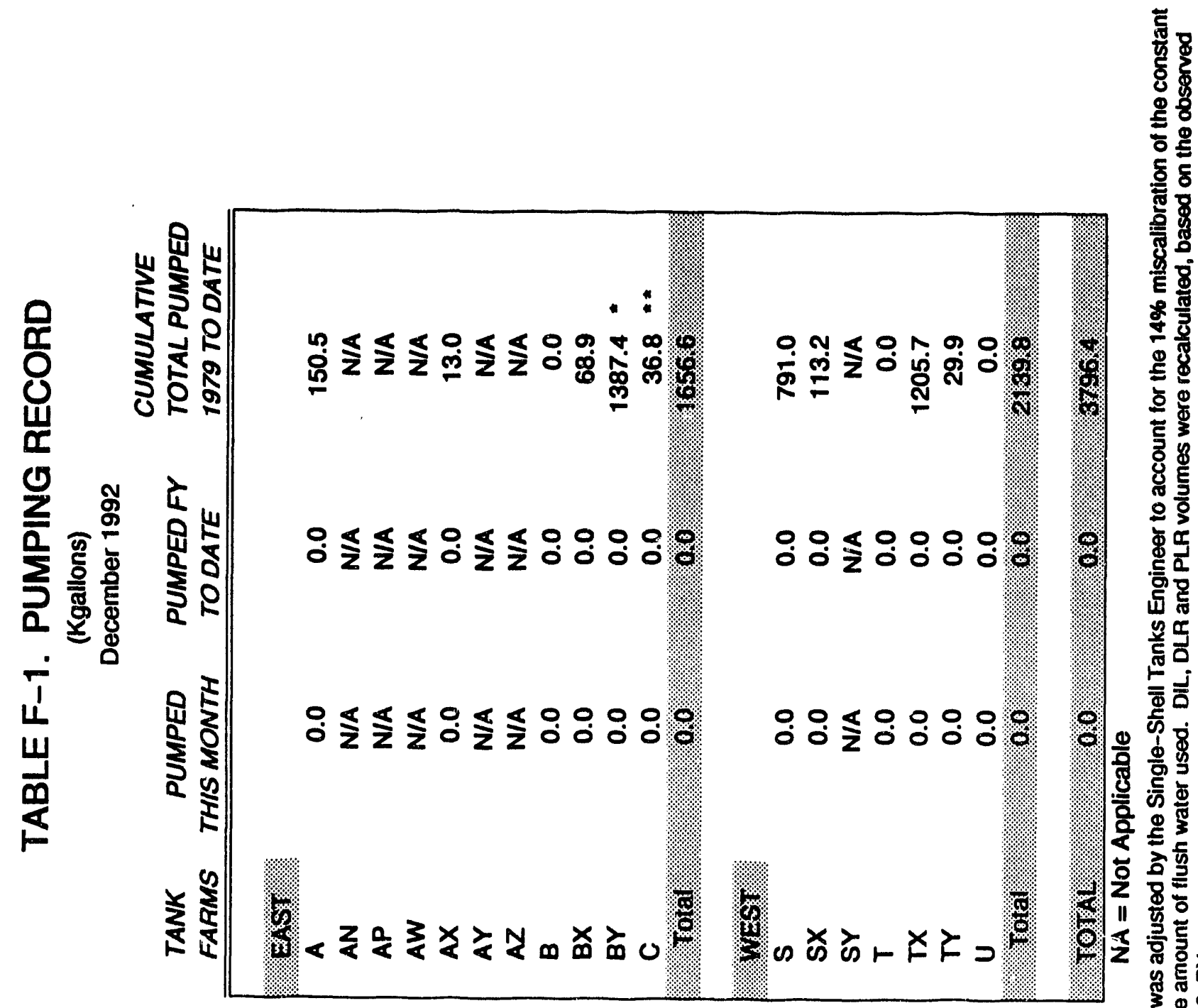


\section{WHC-EP-0182-57}

This page intentionally left blank. 
WHC-EP-0182-57

\section{APPENDIX G}

CATCH TANKS AND SPECIAL SURVEILLANCE FACILITIES

G-1 
WHC-EP-0182-57

This page intentionally left blank. 


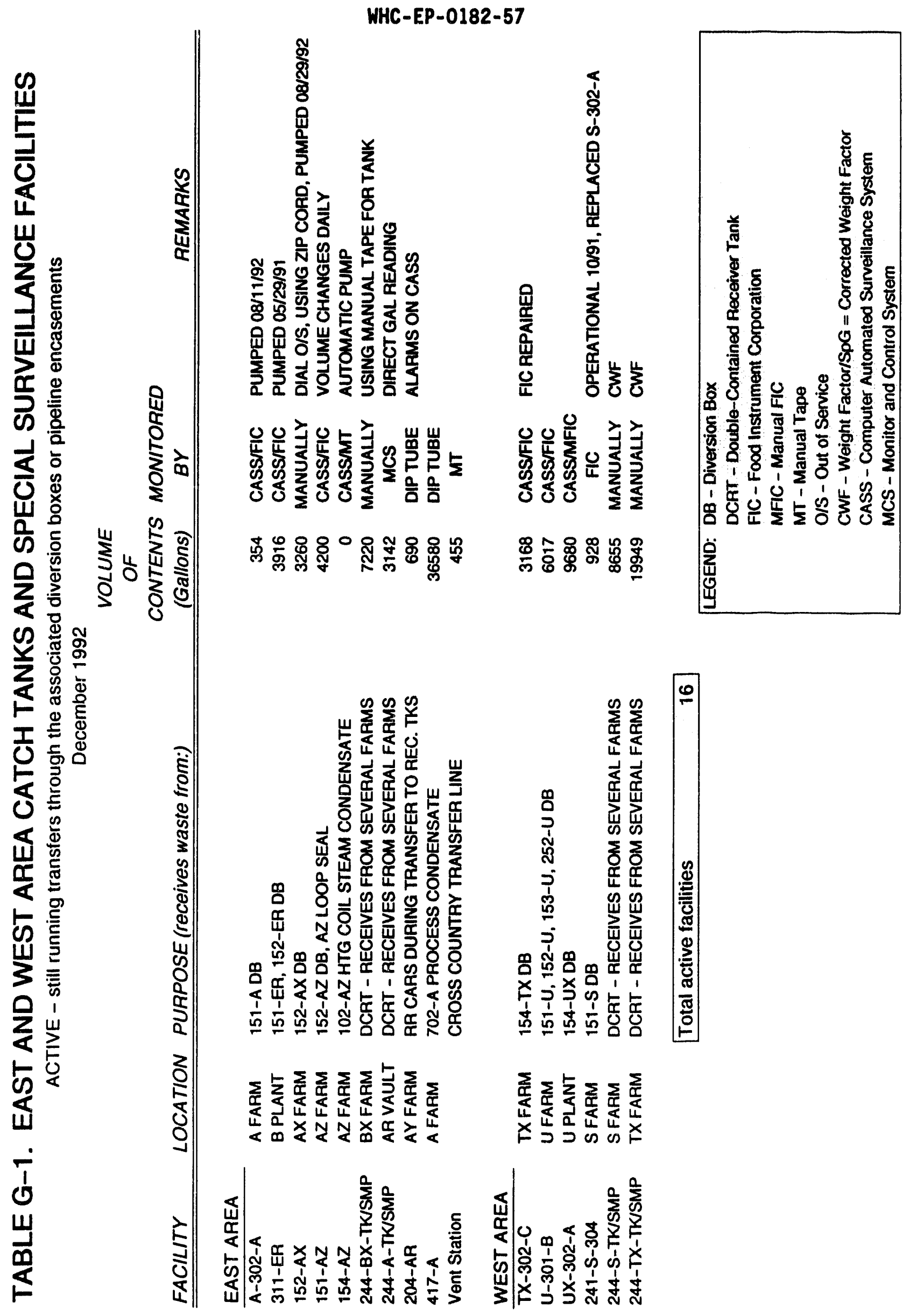


WHC-EP-0182-57
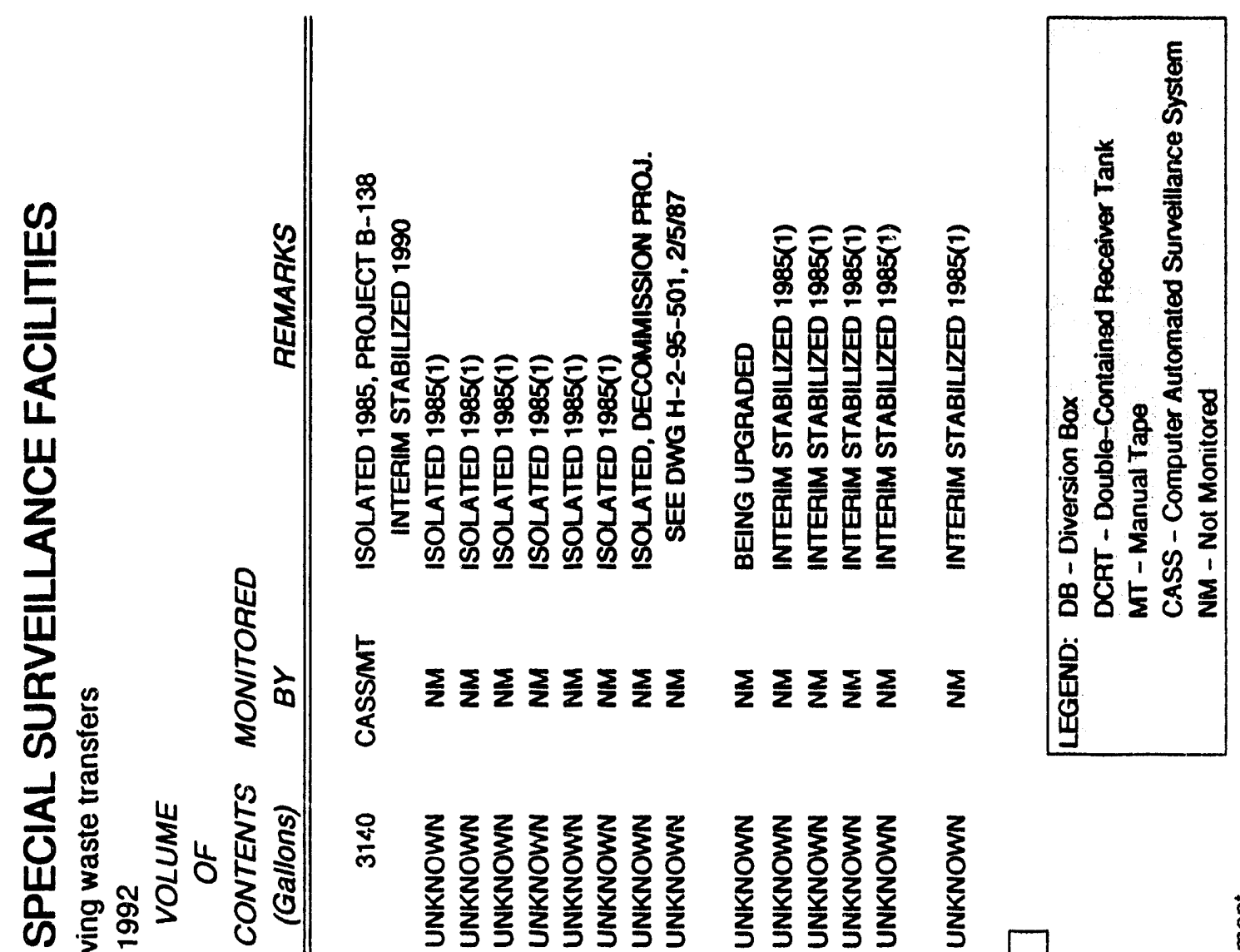

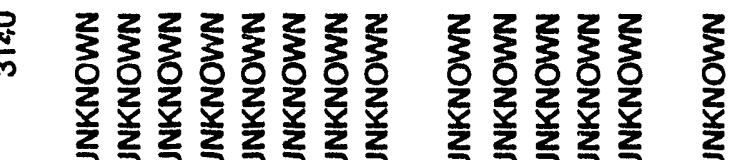

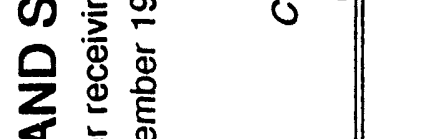

裙

일

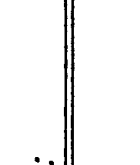

$<$

交

(5)

4

$\frac{1}{\alpha}$

5

岃

i

|

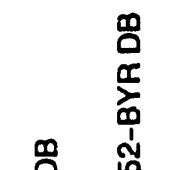

罟

品

กั

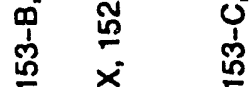

ํํㅁ 总出出

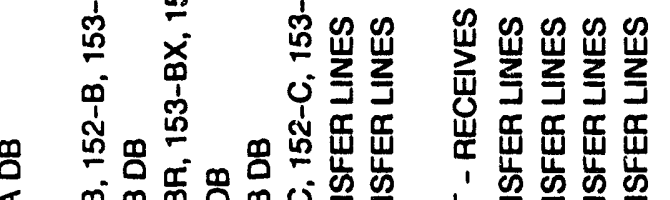

« क ⿴囗十

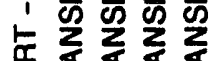

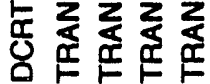

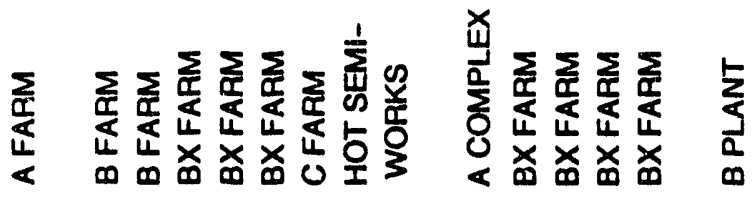

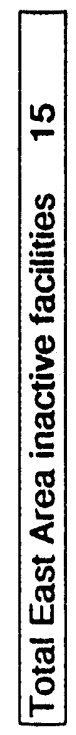

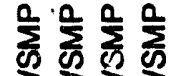

$\frac{1}{5}$

కై

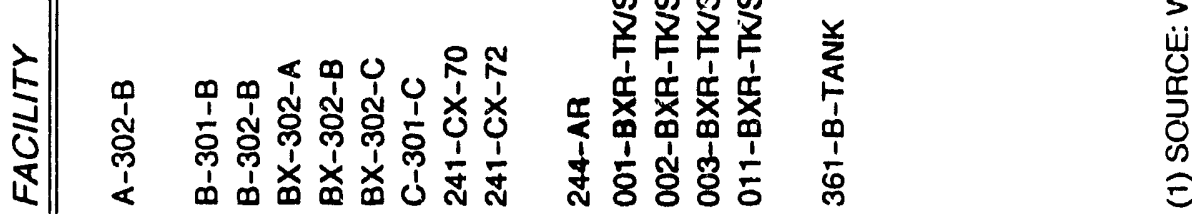


WHC-EP-0182-57

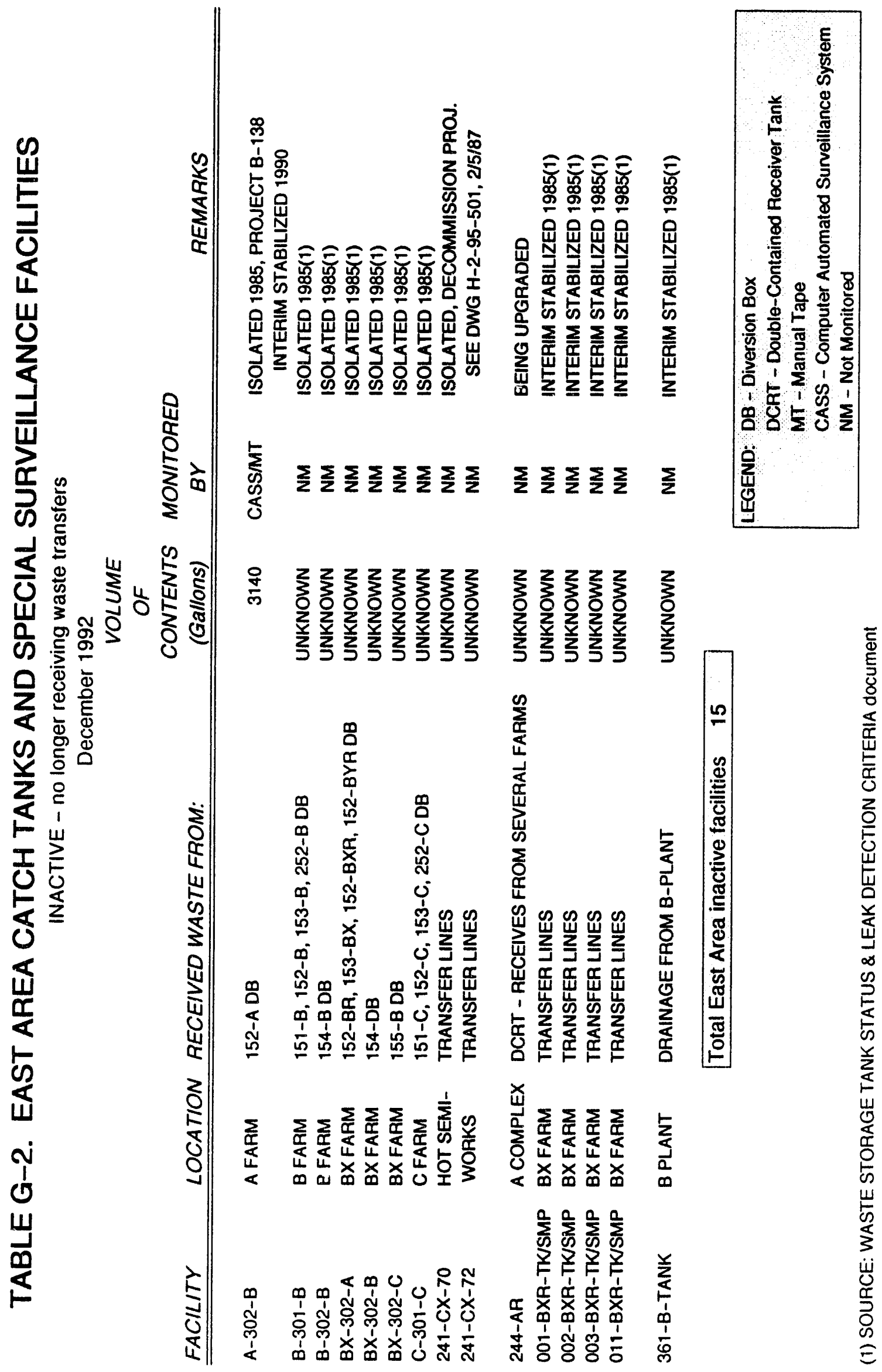


WHC-EP-0182-57

This page intentionally left blank. 
WHC-EP-0182-57

\section{APPENDIX H}

\section{LEAK VOLUME ESTIMATES}


WHC-EP-0182-57

This page intentionally left blank.

$H-2$ 
WHC-EP-0182-57

TABLE H-1. SINGLE-SHELL TANK LEAK VOLUME ESTIMATES (4) (Sheet 1 of 5)

\begin{tabular}{|c|c|c|c|c|c|c|}
\hline \multirow[b]{2}{*}{ Tank No. } & \multirow{2}{*}{$\begin{array}{l}\text { Date Declared } \\
\text { Confirmed or } \\
\text { Assumed Leaker (3) }\end{array}$} & \multirow{2}{*}{$\begin{array}{l}\text { Volume (2) } \\
\text { (Gallons) }\end{array}$} & \multirow{2}{*}{$\begin{array}{l}\text { Associated } \\
\text { KiloCuries } \\
137 \text { cs (11) }\end{array}$} & \multirow{2}{*}{$\begin{array}{l}\text { Interim } \\
\text { Stabilized } \\
\text { Date }\end{array}$} & \multicolumn{2}{|c|}{ Leak Estimate } \\
\hline & & & & & Updated & Reference \\
\hline $\begin{array}{l}241-A-103 \\
241-A-104 \\
241-A-105\end{array}$ & $\begin{array}{l}1987 \\
1975 \\
1963\end{array}$ & $\begin{array}{c}5500 \text { (10) } \\
500102500 \\
100000 \\
277000 \\
2700\end{array}$ & $\begin{array}{l}0.8 \text { to } 1.8 \\
85 \text { to } 760(9)\end{array}$ & $\begin{array}{l}8988 \\
9778 \\
7779\end{array}$ & $\begin{array}{l}1987 \\
1983 \\
1991\end{array}$ & $\begin{array}{l}(a)(a) \\
(b),(c)\end{array}$ \\
\hline $\begin{array}{l}241-A X-102 \\
241-A X-104\end{array}$ & $\begin{array}{l}1988 \\
1977\end{array}$ & $3000\left(\begin{array}{l}(10) \\
(8)\end{array}\right.$ & & $\begin{array}{l}9 / 88 \\
8 / 81\end{array}$ & $\begin{array}{l}1989 \\
1989\end{array}$ & (n) \\
\hline $\begin{array}{l}241-\mathrm{B}-101 \\
241-\mathrm{B}-103 \\
241-\mathrm{B}-105 \\
241-\mathrm{B}-107 \\
241-\mathrm{B}-110\end{array}$ & $\begin{array}{l}1974 \\
1978 \\
1978 \\
1980 \\
1981\end{array}$ & $\left.\begin{array}{r}\bar{z}(8) \\
\bar{z}(8) \\
8000 \\
10000(10) \\
10\end{array}\right)$ & & $\begin{array}{l}3 / 81 \\
2185 \\
12 / 84 \\
3385 \\
12 / 84\end{array}$ & $\begin{array}{l}1989 \\
1989 \\
1989 \\
1986 \\
1986\end{array}$ & $\begin{array}{l}\text { (g) } \\
\text { (d) } \\
\text { (d) }\end{array}$ \\
\hline $\begin{array}{l}241-B-111 \\
241-B-112 \\
241-B-201 \\
241-B-203 \\
241-B-204\end{array}$ & $\begin{array}{l}1978 \\
1978 \\
1980 \\
1983 \\
1984\end{array}$ & $\begin{array}{l}20-0 \\
1200 \\
300 \\
300 \\
400 \\
(10) \\
(10)\end{array}$ & & $\begin{array}{l}685 \\
585 \\
8 / 81 \\
664 \\
6 / 84\end{array}$ & $\begin{array}{l}1989 \\
1989 \\
1984 \\
1986 \\
1989\end{array}$ & (e) \\
\hline $\begin{array}{l}241-8 X-101 \\
241-B X-102 \\
241-8 X-108 \\
241-B X-110 \\
241-B X-111\end{array}$ & $\begin{array}{l}1972 \\
1971 \\
1974 \\
1976 \\
1984\end{array}$ & $\begin{array}{r}70000^{(8)} \\
2500 \\
=-(8)\end{array}$ & 0.58 & $\begin{array}{l}9 / 78 \\
11 / 78 \\
7779 \\
885 \\
\text { N/A }\end{array}$ & $\begin{array}{l}1989 \\
1986 \\
1986 \\
1989 \\
1989\end{array}$ & (8) \\
\hline $\begin{array}{l}241-\mathrm{BY}-103 \\
241-\mathrm{BY}-105 \\
241-\mathrm{BY}-106 \\
241-8 Y-107 \\
241-\mathrm{BY}-108\end{array}$ & $\begin{array}{l}1973 \\
1984 \\
1984 \\
1984 \\
1972\end{array}$ & $\begin{array}{l}<5000 \\
\bar{z}(8) \\
15100 \\
<5000\end{array}$ & & $\begin{array}{l}\text { N/A } \\
\text { N/A } \\
\text { N/A } \\
2 / 85\end{array}$ & $\begin{array}{l}1983 \\
1989 \\
1989 \\
1989 \\
1983\end{array}$ & $\begin{array}{l}\text { (a) } \\
\text { (9) } \\
\text { (9) } \\
\text { (a) }\end{array}$ \\
\hline $\begin{array}{l}241-C-101 \\
241-C-110 \\
24-C-111 \\
241-C-201 \\
241-C-202 \\
241-C-203 \\
241-C-204\end{array}$ & $\begin{array}{l}1980 \\
1984 \\
1968 \\
1988 \\
1988 \\
1984 \\
1988\end{array}$ & $\begin{array}{r}20000(10) \\
2000 \\
5500 \\
5550 \\
450 \\
400 \\
350 \\
350\end{array}$ & & $\begin{array}{l}11 / 83 \\
N / A \\
3 / 84 \\
3 / 82 \\
881 \\
3 / 82 \\
9 / 82\end{array}$ & $\begin{array}{l}1986 \\
1989 \\
1989 \\
1987 \\
1987 \\
1986 \\
1987\end{array}$ & $\begin{array}{l}\text { (d) } \\
\text { (g) } \\
\text { (d) } \\
\text { (d) } \\
\text { (i) }\end{array}$ \\
\hline $241-S-104$ & 1968 & $24000(10)$ & & $12 / 84$ & 1989 & (g) \\
\hline $\begin{array}{l}241-S X-104 \\
241-S X-107 \\
241-S X-108\end{array}$ & $\begin{array}{l}1988 \\
1964 \\
1962\end{array}$ & $\begin{array}{l}6000(10) \\
54000 \text { (10) } \\
2400 \text { to }\end{array}$ & 17 to $140(\mathrm{~m})(\mathrm{q}$ & $\begin{array}{l}\text { N/A } \\
10 / 79 \\
8 / 79\end{array}$ & $\begin{array}{l}988 \\
1983 \\
1991\end{array}$ & 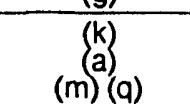 \\
\hline $\begin{array}{l}241-S X-109 \\
241-S X-110\end{array}$ & $\begin{array}{l}1965 \\
1976\end{array}$ & $\begin{array}{r}35000 \\
<10000 \\
5500\end{array}$ & $<40(n)$ & $\begin{array}{l}5 / 81 \\
8 / 79\end{array}$ & $\begin{array}{l}1992 \\
1989\end{array}$ & (n) \\
\hline $\begin{array}{l}241-S x-111 \\
241-S X-112 \\
241-S X-113 \\
241-S X-114 \\
241-S X-115\end{array}$ & $\begin{array}{l}1974 \\
1969 \\
1962 \\
1972 \\
1965\end{array}$ & $\begin{array}{c}500102000 \\
30000 \\
15000 \\
50000\end{array}$ & 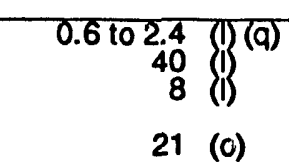 & $\begin{array}{l}7779 \\
7779 \\
1178 \\
7779 \\
9 / 78\end{array}$ & $\begin{array}{l}1986 \\
1986 \\
1986 \\
1989 \\
1992\end{array}$ & 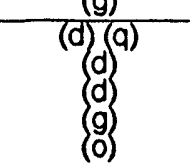 \\
\hline $\begin{array}{l}241-T-101 \\
241-T-103 \\
241-T-106 \\
241-T-107 \\
241-T-108 \\
241-T-109 \\
241-T-111\end{array}$ & $\begin{array}{l}1992 \\
1974 \\
1973 \\
1984 \\
1974 \\
1974 \\
1984\end{array}$ & $\begin{array}{r}7500(10) \\
<1000(10) \\
115000(10) \\
<1000(10) \\
<1000(10) \\
<1000(10)\end{array}$ & 40 (I) & $\begin{array}{l}\text { N/A } \\
11 / 83 \\
8 / 81 \\
N / A \\
11 / 78 \\
12 / 84 \\
N / A\end{array}$ & $\begin{array}{l}1992 \\
1989 \\
1986 \\
1989 \\
1980 \\
1989 \\
1980\end{array}$ & $\begin{array}{l}\text { (p) } \\
\text { (p) } \\
\text { (9) } \\
\text { (1) } \\
\text { (9) }\end{array}$ \\
\hline $\begin{array}{l}241-T X-105 \\
241-T X-107 \\
241-T X-11 \\
241-T X-113 \\
241-T X-114 \\
241-T X-115 \\
241-T X-116 \\
241-T X-117\end{array}$ & $\begin{array}{l}1977 \\
1984 \\
1977 \\
1974 \\
1974 \\
1977 \\
1977 \\
1977\end{array}$ & 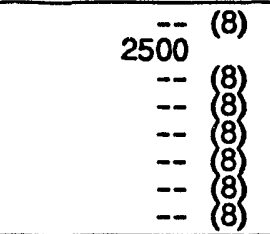 & & $\begin{array}{l}9 / 83 \\
10 / 79 \\
4 / 83 \\
4 / 83 \\
4 / 83 \\
9 / 83 \\
4483 \\
3 / 83\end{array}$ & $\begin{array}{l}1989 \\
1986 \\
1989 \\
1989 \\
1989 \\
1989 \\
1989 \\
1989\end{array}$ & $\begin{array}{l}\text { (g) } \\
\text { (g) } \\
\text { g9 } \\
\text { g9 } \\
\text { g9 } \\
\text { (9) }\end{array}$ \\
\hline $\begin{array}{l}241-T Y-101 \\
241-T Y-103 \\
241-T-104 \\
241-T Y-105 \\
241-T Y-106\end{array}$ & $\begin{array}{l}1973 \\
1973 \\
1981 \\
1960 \\
1959\end{array}$ & $\begin{array}{l}<1000(10) \\
3000 \\
1400 \\
35000 \\
20000\end{array}$ & $\begin{aligned} 0.7 & \text { (1) } \\
4 & \text { (1) }\end{aligned}$ & $\begin{array}{l}8 / 83 \\
2183 \\
1183 \\
2283 \\
11 / 78\end{array}$ & $\begin{array}{l}1980 \\
1986 \\
1986 \\
1986 \\
1986\end{array}$ & (d) \\
\hline $\begin{array}{ll}241-U-101 & \\
241-U-104 & (7) \\
241-U-110 & \\
241-U-112 & (7) \\
\end{array}$ & $\begin{array}{l}1959 \\
1961 \\
1975 \\
1980 \\
\end{array}$ & $\begin{array}{r}30000 \\
55000 \\
5000108100 \quad(10) \\
8500(10) \\
\end{array}$ & $\begin{array}{l}20 \\
0.09 \\
0.05(3)\end{array}$ & $\begin{array}{l}9 / 79 \\
10 / 78 \\
12 / 84 \\
9 / 78 \\
\end{array}$ & $\begin{array}{l}1986 \\
1986 \\
1986 \\
1986 \\
\end{array}$ & (d) (d) \\
\hline 67 Tanks. & & 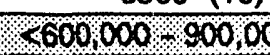 & & & & \\
\hline
\end{tabular}

N/A = not applicable (not yet interim stabilized)

FOOTNOTES: SEE NEXT PAGE 
WHC-EP-0182-57

TABLE H-1. Single-Shell Tank Leak Volume Estimates

(Sheet 2 of 5 )

Footnotes:

(1) Current estimates (see reference b) are that $610 \mathrm{Kgal}$ of cooling water was added to Tank 241-A-105 from November 1970 to December 1978 to aid in evaporative cooling. In accordance with Dangerous Waste Regulations (Washington Administrative Code 173-303-070 (2)(a)(ii), as amended, Washington State Department of Ecology, 1990, 01ympia, Washington), any of this cooling water that has been added and subsequently leaked from the tank must be classified as a waste and should be included in the total leak volume. In August 1991, the leak volume estimate for this tank was updated and moved into compliance with the WAC regulations. Previous estimates excluded the cooling water leaks from the total leak volume estimates because the waste content (concentration) in the cooling water which leaked should be much less than the original liquid waste in the tank (the sludge is relatively insoluble). The total leak volume estimate in this report (10 Kgal to $277 \mathrm{Kgal}$ ) is based on the following (see References).

1. Reference (b) contains an estimate of $5 \mathrm{Kgal}$ to $15 \mathrm{Kgal}$ for the initial leak prior to August 1968.

2. Reference (b) contains an estimate of $5 \mathrm{Kgal}$ to $30 \mathrm{Kgal}$ for the leak while the tank was being sluiced from August 1968 to November 1970.

3. Reference (b) contains an estimate of $610 \mathrm{Kgal}$ of cooling water added to the tank from November 1970 to December 1978 but it was estimated that the leakage was small during this period. This reference contains the statement "Sufficient heat was generated in the tank to evaporate most, and perhaps nearly all, of this water." This results in a low estimate of zero gallons leakage from November 1970 to December 1978.

4. Reference (c) contains an estimate that 378 to $410 \mathrm{Kgal}$ evaporated out of the tank from November 1970 to December 1978. Subtracting the minimum evaporation estimate from the cooling water added estimate provides a range from 0 to $232 \mathrm{Kgal}$ of cooling water leakage from November 1970 to December 1978.

Low Estimate High Estimate

Prior to August 1968

5,000

15,000

August 1968 to November 1970

November 1970 to December 1978

Totals

$$
5,000
$$

30,000

$\frac{0}{10,000}$

$\frac{232,000}{277,000}$

(2) These leak volume estimates do not include (with some exceptions), such things as: (a) cooling/raw water leaks, (b) intrusions (rain infiltration) and subsequent leaks, (c) leaks inside the tank farm but not through the tank liner (surface leaks, pipeline leaks, leaks at the joint for the overflow or fill lines, etc.), and (d) leaks from catch tanks, diversion boxes, encasements, etc. 


\section{WHC-EP-0182-57}

\section{TABLE H-1. Single-She11 Tank Leak Volume Estimates}

(Sheet 3 of 5 )

(3) In many cases, a leak was suspected long before it was identified or confirmed. In 1984, the criteria designations of "suspected leaker," "questionable integrity," "confirmed leaker," "declared leaker," "borderline," and "dormant," were merged into one category now reported as "assumed leaker." See reference (f) for explanation of when, how long, and how fast some of the tanks leaked.

(4) There is an effort currently in progress to reevaluate these leak volume estimates. During the FY 1993 funding reviews, this reevaluation of leak volumes was given a priority which resulted in this activity no longer being funded. The priority versus funding will be reevaluated prior to FY 1994.

(5) The leak volume estimate date for these tanks is before the "declared leaker" date because the tank was in a "suspected leaker" or "questionable integrity" status; however, a leak volume had been estimated prior to the tank being reclassified.

(6) The increasing radiation levels in drywells and laterals associated with these three tanks could be indicative of a continuing leak or movement of existing radionuclides in the soil. There is no conclusive way to confirm these observations.

(7) These four tanks also show slight indications of continuing leaks or movement of radionuclides in the soil.

(8) Methods were used to estimate the leak volumes from these 19 tanks based on the assumption that their cumulative leakage is approximately the same as for 18 of the 24 tanks identified in footnote (10). For more details see reference (g). The total leak volume estimate for these tanks is $150 \mathrm{Kgal}$ (rounded to the nearest $10 \mathrm{Kgal}$ ), for an average of approximately $8 \mathrm{Kgal}$ for each of the 19 tanks.

(9) The total has been rounded to the nearest $50 \mathrm{Kgal}$. Upperbound values were used in many cases in developing these estimates. It is likely that some of these tanks have not actually leaked.

(10) Leak volume estimate is based solely on observed liquid level decreases in these tanks. This is considered to be the most accurate method for estimating leak volumes.

(11) The curie content listed is as listed in the reference document and is not decayed to a consistent date; therefore, a cumulative total is inappropriate. 
TABLE H-1. Single-Shel1 Tank Leak Volume Estimates.

(Sheet 4 of 5 )

References:

(a) Murthy, K.S., et a1, June 1983, Assessment of Single-She 11 Tank Residual Liquid Issues at Hanford Site, Washington, PNL-4688, Pacific Northwest Laboratory, Richland, Washington.

(b) WHC, 1991a, Tank 241-A-105 Leak Assessment, WHC-MR-0264, Westinghouse Hanford Company, Richland, Washington.

(c) WHC, 1991b, Tank 241-A-105 Evaporation Estimate 1970 Through 1978, WHC-EP-0410, Westinghouse Hanford Company, Richland, Washington.

(d) Smith, D. A., January 1986, Single-Shell Tank Isolation Safety Analysis Report, SD-WM-SAR-006, Rev. 1, Westinghouse Hanford Company, Richland, Washington.

(e) McCann, D. C., and T. S. Vail, September 1984, Waste Status Summary, RHO-RE-SR-14, Rockwell Hanford Operations, Richland, Washington.

(f) Catlin, R. J., March 1980, Assessment of the Surveillance Program of the High-Level Waste Storage Tanks at Hanford, Hanford Engineering Development Laboratory, Richland, Washington.

(g) Baumhardt, R. J., May 15, 1989, Letter to R. E. Gerton, U.S. Department of Energy-Richland Operations Office, Single-Shell Tank Leak Volumes, Westinghouse Hanford Company, Richland, Washington.

(h) WHC, 1990a, Occurrence Report "Surface Level Measurement Decrease in Single-She!1 Tank 241-AX-102," WHC-U0-89-023-TF-05, Westinghouse Hanford Company, Richland, Washington.

(i) Groth, D. R., July 1, 1987, Internal Memorandum to R. J. Baumhardt, Liquid Leve1 Losses in Tanks 241-C-201, -202 and -204, 65950-87-517, Westinghouse Hanford Company, Richland, Washington.

(j) Groth, D. R. and G. C. Owens, May 15, 1987, Internal Memorandum to J. H. Roecker, Tank 103-A Integrity Evaluation, Westinghouse Hanford Company, Richland, Washington.

(k) Campbe11, G. D., July 8, 1988, Internal Memorandum to R. K. Welty, Engineering Investigation: Interstitial Liquid Level Decrease in Tank 241-SX-104, 13331-88-416, Westinghouse Hanford Company, Richland, Washington.

(1) ERDA, 1975, Final Environmental Statement Waste Management Operations, Hanford Reservation, Richland, Washington, ERDA-1538, 2 vols., U.S. Energy Research and Development Administration, Washington, D.C.

(m) WHC, 1992a, Tank 241-SX-108 Leak Assessment, WHC-MR-0300, Westinghouse Hanford Company, Richland, Washington.

(n) WHC, 1992b, Tank 241-SX-109 Leak Assessment, WHC-MR-0301, Westinghouse Hanford Company, Richland, Washington. 
TABLE H-1. Single-Shell Tank Leak Volume Estimates.

(Sheet 5 of 5 )

(0) WHC, 1992c, Tank 241-SX-115 Leak Assessment, WHC-MR-0302, West inghouse Hanford Company, Richland, Washington.

(p) WHC, 1992d, Occurrence Report, "Apparent Decrease in Liquid Level in Single She11 Underground Storage Tank 241-T-101, Leak Suspected; Investigation Continuing," RL-WHC-TANKFARM-1992-0073, Westinghouse Hanford Company, Richland, Washington.

(q) WHC-1990b, A History of the 200 Area Tank Farms, WHC-MR-0132, Hestinghouse Hanford Company, Richland, Washington. 
WHC-EP-0182-57

This page intentionally left blank. 


\section{DISTRIBUTION}

Number of copies

OFFSITE - USA

20

U. S. Department of Energy-Headquarters

1000 Independence Avenue, SW

Washington, D. C. 20585

Emile Bernard

$E M-50$

H. Calley

H. Eckart

Teresa Fryberger

Sherry Gibson

A. Griffith

L. Gunn

D. Gupta

J. S. Kang

Kenneth Lang

J. C. Lehr

G. Mellinger

John Morrey

C. O'Dell

C. Pepson

J. C. Tseng

S. Woodbury

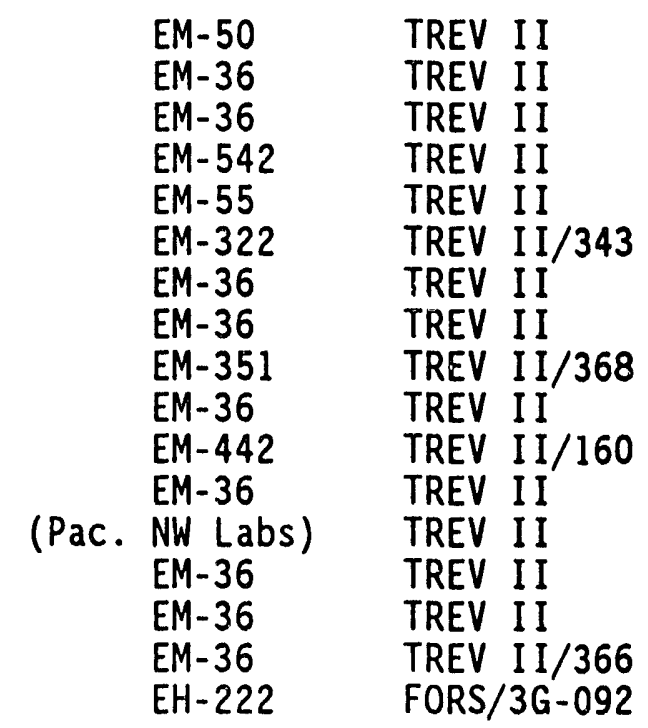

19901 Germantown Rd, Germantown, MD 20585
R. Lasky
EH-32.1
GTN/G-115
J. Psaras
NS-20 GTN
P. Worthington
NS-20 GTN

1

$U$. S. Department of Energy - Oak Ridge Operations office

P. 0. Box 2001

Oak Ridge, TN 37831

W. D. Adams EW-40

U. S. Department of Energy - Savannah River Site

P. 0. Box A

Aiken, SC 29808

C. Anderson

$707-\mathrm{H}$

Michael Chandler

$703-\mathrm{H}$

Mazen Shurrab

$704-\mathrm{H}$

T. C. Temple

L. Sjostrom

W. K. West

$V$. Wheeler

$704-8 \mathrm{H}$

$704-S$ 
WHC-EP-0182-57

Distribution - continued

1

U. S. Department of Energy - Idaho Operations

785 D. 0. E. Place

Idaho Falls, ID 83402

W. Sato

MS-11-18

1

U. S. Environmental Protection Agency

Region 10

712 Swift Boulevard, Suite 5

Richland, WA 99352

P. T. Day

Washington State Department of Ecology

Nuclear \& Mixed Waste Management

719 Sleater-Kinney Road S. E., Suite 200

Lacey, WA 98503-1138

R. Stanley

M. T. Gordon

7601 W. Clearwater \#102

Kennewick, WA 99336

Greta Davis

1

Washington State Department of Health Radiation Protection Section

Industrial Park Building 5, LE-13

01 ympia, WA 98504

A. Conklin

Oregon State Department of Energy 625 Marion St. N.E.

Salem, OR 97310

Janet Franco

$1 \quad$ Oregon State Water Resources Department

Ground Water Hanford Studies

3850 Portland Road

Salem, OR 97310

R. 0. Patt

1 Lawrence Livermore National Laboratory

Box 808, East Avenue

Livermore, CA 94550

B. C. Hudson

$L-221$

Distr-2 
WHC-EP-0182-57

\section{Distribution - continued}

Oak Ridge National Laboratory

P. 0. Box 2009

Oak Ridge, TN 37831-6385

C. Forsberg

MS-6495

T. S. Kness

MS -8088

B1dg 9.08

Chemical Technology Division

P. 0. Box 2008

Emory D. Collins

Los Alamos National Laboratory

F. 0. Box 1663

Los Alamos, NM 87545

Stephen Agnew

Group INC-14

T. Larson

C -346

Sylvia Lee

A. Nuels

C -915

H. Sullivan

$\mathrm{K}-557$

$N-6$

$N-6$

Brookhaven National Laboratory 50 Rutherford

Upton, NY 11973

K. K. Bandyopadhyay, Bldg 475-C

M. Reich, Bldg 475-C

P. D. Kalb, Bldg. 703

Brookhaven National Laboratory 1409 Jan Drive

Wilmington, DE 19803

Michael Streicher

Sandia National Laboratories

1515 Eubank, NE

P. 0. Box 58no

Albuquerque, NM 87185

Scott Slezak, Division 6402

Leon D. Chapman, Program Manager

Industrial Waste Reduction Program

Massachusetts Institute of Technology

77 Massachusetts Avenue

Cambridge, MA 02139

Mujid S. Kazimi

Professor and Head

Department of Nuclear Engineering

Distr-3 
WHC-EP-0182-57

Distribution - continued

BDM International. Inc.

20030 Century Blvd, Suite 101

Germantown, MD 20874

P. Kiang

K. J. Mahoney

2

SAIC

Trevion 1, Suite 300

12850 Middlebrook Rd.

Germantown, MD 20874

J. Bunting

J. R. Pearring

1

102 Windham Road

Oak Ridge, TN 37830

D. 0. Campbe11

1

SAIC

1845 Terminal Drive, Suite 130

Richland, WA 99352

J. Mishima

1

Harvard School of Public Health

665 Huntington Avenue

Boston, MA 02115

M. First

1

Confederated Tribes, Umatilla Indian Reservation

P. 0. Box 638

Pendleton, OR 97801

Rick George

4

West Valley Nuclear Services Co.

P. 0. Box 191

West Valley, NY 14171

S. Ketola

D. K. Ploetz

MS -191

Ram Shukla

Don Stroud

MS -305

\section{General Accounting Office}

P. 0. Box 321

?ichland, WA 99352

C. R. Abraham 
WHC-EP-0182-57

Distribution - continued

Defense Nuclear Facilities Safety Board

625 Indiana Ave, N. W., Suite 700

Washington; D. C. 20004

Dan Burnfield

Lester Clemons

Paul Gubanc

Suite 700

Dermot M. Winters, Geological Engineer

Westinghouse Idaho Nuclear Corporation

P. 0. Box 4000

Idaho Falls, ID 83403-4000

B. Griebenow

MS -5104

A. P. Hoskins

MS -5217

1

C. Abrams

1987 Virginia Drive

Idaho Falls, ID 83404

1

F. Carlson

6965 North, 5th West

Idaho Falls, ID 83401

Joseph J. DiNunno, Engineering Consultant

44 Carriage Lane

Annapolis, MD 21401

1

D. Oakley

40912 th Street, SW, \#310

Washington, D. C. 20024

1

Dr. A. Veletsos

Department of Civil Engineering

Rice University

P. 0. Box 1892

Houston, TX 77252

Westinghouse Materials Company of Ohio

P. 0. Box 398704

Cincinnati $\mathrm{OH} 45239-8704$

David L. Jacoboski

Senior Engineer, Technology Demonstration

Hestinghouse Savannah River Company

P. 0. Box 616

Aiken, SC 29802

P. d'Entremont 
WHC-EP-0182-57

Distribution - continued

Institute for Energy and Environmental Reseach

6935 Laurel Avenue

Takoma Park, MD 20912

Dr. Arjun Makhijani, President

1

SPAR Aerospace Ltd.

20 Avon Meadow Lane, Suite 220

Avon, CT 06001

Peter W. Kruse

Advanced Technology Systems Division

1

Redzone Robotics, Inc.

2425 Liberty Ave

Pittsburgh, PA 15222-4639

David W. White

National Research Council. National Academy of Sciences 2101 Constitution Ave., N. W. Washington D. C. 20418

Robert S. Andrews, Senior Staff officer

Board on Radioactive Waste Management

Converse Consultants

18 W. Mercer Street, Suite 300

Seattle, WA 98119

David Stanley

1

Brown \& Caldwell

100 W. Harrison

Seattle, WA 98119

Hal Cooper

1

BOVAY Northwest Inc.

660 Swift, Suite D

Richland, WA 99352

T. J. MCLaught in

Omega Environmental Technology

655 Montgomery Street, Suite 1000

San Francisco, CA 94111

Mike Bailey 
WHC-EP-0182-57

\section{Distribution - continued}

GEC ALSTHOM Engineering Systems

P. 0. Box 1274

Richland, WA 99352

J. W. Riddington,

Vice President, Nuclear Marketing

1

T. S. Elleman

North Carolina State University

Department of Nuclear Energy

P. 0. Box 7909

Raleigh, NC 27606

1

Mike Lingle

Stone \& Webster

7677 E. Berry Ave

Englewood, CA 80111

1

Bryant Mather

Corps of Engineers

WESSV - Z

3909 Halls Ferry Rd

Vicksburg, MS 39180-6199

1

Paul Shewmon

Prof. Metallurgical Engineer

Ohio State University

2477 Lytham Road

Columbus, $\mathrm{OH} 43220$

\section{Waste Management External Advisory Committee Members}

Dr. Frank L. Parker

Professor of Environmental and Water Resources Engineering Vanderbilt University

P. 0. Box 1596, Station B

Nashville, TN 37235

Dr. Bruce R. Kowalski

Professor of Chemistry, Co-director of Center for Process

Analytical Chemistry

University of Washington

Chemistry Department, B1dg 10

Seattle, WA 98195

Dr. Greg R. Choppin

Professor of Chemistry

Florida State University

Department of Chemistry, B-164

Tallahassee, FL 32306 
WHC-EP-0182-57

\section{Distribution - continued}

Dr. Chester Grelecki

President, Chief Scientist

Hazards Research Corporation

200 Valley Road

Mt. Arlington, NJ 07856

Dr. Alfred Schneider

MIT Department of Nuclear Engineering

Room 24-1098

77 Massachusetts Avenue

Cambridge, MA 02139

Dr. Gary Powers

President

Design Science, Inc.

163 Witherow Road

Sewickley, PA 15143

1

Ames Laboratory

7 Spedding $\mathrm{Hall}$

Iowa State University

Ames, IA 50011

Bill Haas

1

RKK Ltd.

16404 Smokey Pt. B1vd, Suite 303

Arlington, WA 98223

Chris Reno

1

MACTEC

8320 Centerbrook Place

Alexandria, VA 22308

Stan Blacker

1

Engineering-Science, Inc.

1955 Jadwin Ave, Suite 470

Richl and, WA 99352

Matt Sakach

1 EBASCO Services, Inc.

1201 Jadwin Avenue, Suite 202

Richland, WA 99352-3429

F. J. Young

1

MTL Systems, Inc.

3481 Dayton-Xenia Road

Dayton, $\mathrm{OH}$ 45431-0299

E. McDaniel

Distr-8 


\section{WHC-EP-0182-57 \\ Distribution - continued}

1

Nuclear Consulting Services, Inc. 7000 Huntley Road

P. 0. Box 29151

Columbus, $\mathrm{OH} 43229$

Dr. J. Louis Kovach

$1 \quad$ Battelle Laboratories

$505 \mathrm{King}$ Avenue

Columbus, $\mathrm{OH}$ 43201-2693

Rob Taylor Jr., P.E.

Rm 13-6-016

1 Portland General Electric Co.

121 S. W. Salmon St.

Portland, OR 97204-2991

Wayne Lei, 3WTCBR05

\section{OFFSITE - FOREIGN}

1

British Nuclear Fuels Ltd

Risley Warrington

Cheshire WA3 6AS

United Kingdom

Howard A. Edwards

1

Ricardo Hitec Ltd

Club street Works, Bamber Bridge

Preston, PR5 6FN

United Kingdom

P. K. J. Smith

1

Telerobot

Consorzio Telerobot

Via Hermada 6

16154 Genova, Italy

Bruno Sessarego

1

SGN

1 , rue des Herons, Montigny-C-Bretonneux

78132 Saint-Quentin-en-Yvel ines Cedex,

France

Serge Merlin 
WHC-EP-0182-57

\section{Distribution - continued}

2

CEA - Saclay

DCC/DIR

$\mathrm{Ba}^{\prime} \mathrm{t} 121$

91190 GIF/Yvette Cedex

France

G. Baudin

R. Atabek

1

Hans Wal ischmiller GmbH

D-7778 Markdorf/Bodensee

Germany

Wolfgang Wal ischmiller

ONSITE

24

U. S. Department of Energy-Richland Operations Office

G. E. Bishop

$R 2-62$

K. W. Bracken

A5- 16

G. J. Bracken

R4-02

S. T. Burnum

A5-16

N. R. Croskrey

$\mathrm{R} 1-30$

R. C. Cullison

A5 -55

J. J. Davis

A5-16

L. Erickson

A5-16

R. E. Gerton

A4-02

W. F. Hendrickson

A4-02

R. D. Hildebrand

A5- 55

P. E. LaMont

A5 -16

B. L. Nicoll

A5-16

T. Nobie

A4-02

R. H. Pestes

A5-16

L. E. Petersen

A5 -10

G. W. Rosenwald

A4-02

G. H. Sanders

A5-16

J. B. Sullivan

A. $5-10$

A. D. Toth

R2-62

W. R. Wresinski

A5 -16

J. K. Yerxa

A5 - 15

Reading Room

Al -65

\section{Kaiser Engineering Hanford}
C. J. Denson
E6-51
J. E. Fasso Jr.
E2-80
D. J. Shrimpton
E6-21

Stone \& Webster Engineering Co.

E. L. Richards

A4-35

Distr-10 
WHC-EP-0182-57

Distribution - continued

1

MACTEC

J. Janus

G6-18

21

Pacific Northwest Laboratories

D. B. Baird

K7 - 54

D. W. Bennett

K5-22

S. A. Bryan

P7 -25

L. L. Burger

P7 -25

J. F. Fletcher

K7-97

L. K. Holton Jr.

P7 -43

J. Janata

K2-12

B. M. Johnson

$\mathrm{K} 1-78$

$E$. O. Jones

P8-38

L. G. Morgan

P7 -35

B. E. Opitz

K6-81

M. S. Peffers

K7-94

W. G. Richmond

P7 -41

R. D. Scheele

P7-25

P. A. Scott

P7- 19

J. C. Spanner

K2-05

D. Strachan

$K 2-38$

K. L. Steinmaus

K6-84

R. S. Wegeng

K7-97

P. D. Whitney

K7-34

T. W. Wood

$\mathrm{K} 6-47$

Westinghouse Hanford Company
A. T. Alstad
R1-49
R. P. Anantatmula
R2-11
J. D. Anderson
J. N. Appel
I. J. Austin
H. Babad
N3-11
S4-58
T4-01
R2-08
A. D. Bates
T6- 07
P. K. Bhatia
S4-58
T. J. Bander
$\mathrm{HO}-33$
L. L. Barry
Rl-67
G. D. Bazinet
D. L. Becker
L4-71
H5-57
D. B. Bechtold
T6-50
K. H. Bergsman
L6-24
M. V. Berriochoa
B3 -30
D. L. Bjorklund
S6-01
R. J. Bl anchard
R1-17
S1 -57
D. C. Board
H5- 49
R2-11
G. L. Borsheim
R1 -49
V. C. Boyles
L5- 65
H. R. Brager
D. R. Bratzel
L5-31
R. G. Brown
R2-14 
R. K. Brown

J. G. Burk, Jr.

J. H. Bussell

J. A. Caggiano Jr

K. G. Carothers

J. W. Carey

R. J. Cash

G. Christensen

J. C. Conner

F. M. Coony

W. L. Cowley

N. R. Croskrey

G. M. Crumme1

J. M. Cruse

D. S. Cunn ingham

L. T. Cunningham

J. I. Dearing

C. Defigh-Price

T. A. Demitruk

D. R. Dickinson

E. C. Ladd

R. A. Dodd

M. R. Duncan

G. L. Dunford

W. S. Dunnivant

J. A. Eacker

D. R. Ellingson

M. F. Erhart

S. D. Estey

W. G. Farley

K. 0. Fein

R. A. Flores

L. A. Fort

K. D. Fowler

G. L. Fox, Jr.

G. T. Frater

J. R. Freeman-Pollard

J. C. Fulton

K. A. Gasper

G. J. Gauck

R. L. Gilchrist

D. A. Gilles

S. D. Godfrey

D. J. Green

J. M. Grigsby

V. W. Hall

C. S. Haller

D. G. Hamrick

K. L. Hampsten

B. M. Hanion (30)

J. M. Hanson

H. D. Harmon

J. P. Harris III

J. M. Henderson
H5- 68

B3- 25

L7 -06

H5- 29

R1 -51

SO-01

R2-32

H4 -21

H5-31

H6- 07

H5-31

R] -5$]$

R1-51

L5- 63

SO-02

R1-51

H5-72

R2-31

H5-36

L5-31

R1- 19

R1-51

L6-10

R1-51

R2-88

R1-51

B5- 35

R2-11

R2-11

H5- 32

H5-34

So- 12

B2-20

R2-11

L5-01

R1-51

H6- 03

R2-31

R2-08

R2-07

L5- 63

S2-70

R1-51

H5-53

H4 -62

B1-59

R2-18

R1-51

L4 - 71

R1-80

R2 - 40

R2-52

S4-55

S4-55 
WHC-EP-0182-57

\section{Distribution - continued}

D. W. Hendrickson

N. A. Hertelendy

E. G. Hess

M. C. Higginson

J. G. Hili

B. M. Hisaw

J. D. Hopkins

B. K. Horsager

R. D. House

J. H. Huber

J. L. Huckaby

L. L. Humphreys

J. E. Hysjulien

J. E. Irwin

M. N. I I lam

G. D. Johnson

J. L. Juette

L. J. Julyk

R. A. Kirkbride

N. W. Kirch

W. L. Knecht

E. M. Koellermeier

G. M. Koreski

A. G. Krasopoulos

M. Kummerer

M. J. Kupfer

J. E. Langdon

D. L. Lenseigne

D. C. Lini

P. J. Mackey

G. T. MacLean

M. K. Mahaffey

R. M. Marusich

V. D. Maupin

T. B. McCall

J. D. McCormack

K. S. McCullough

M. E. McDonald

T. E. Mensinger

N. J. Milliken

W. J. Millsap

G. J. Miskho

J. R. Mobley

K. L. Morris

J. P. Mullally

L. D. Muhlestein

A. F. Noonan

T. W. Oden

P. C. Ohl

D. B. Pabst

R. B. Pan

I. G. Papp

G. L. Parsons

M. A. Payne
R4-03

H5- 09

R3-09

A4 -25

R2-12

R1 -62

R2-11

S4-57

R2 - 83

RI -49

R2-11

R2 -50

SO-09

B1 -59

R3-08

R2 -78

G6-56

H5-56

S4-58

R2-11

HO- 34

R4-40

R1-51

A5-55

H5-32

H5- 49

S2-96

R2-75

H5- 49

B3-15

S4 - 58

L4-73

H5-32

R1-51

HO- 33

โ5-31

H5-34

R1-62

T3-10

H5-34

H5- 68

R2 - 50

R2-18

H5- 09

S4-57

N1-28

R2 - 12

D2-29

R1-30

B2-35

H5- 53

R3-45

S4-57

R2-50 


\section{Distribution - continued}

T. B. Powers

$\mathrm{HO}-31$

R. K. P'Pool

$\mathrm{T} 1-30$

J. G. Propson

R2-18

T. E. Rainey

R. E. Raymond (2)

R1-49

R. W. Reed

R1-80

I. E. Reep

$R 1-51$

M. A. Rezvani

R2-08

D. Richardson

H5 -55

J. H. Roecker

R2-31

G6-02

L. Ruffin

J. A. Ryan

R2-95

C. C. Scaief

H5- 55

F. A. Schmorde

L7 -06

J. S. Schofield

R2-88

R1-51

C. P. Schroeder

L7-06

D. D. Scott

K. V. Scott

L6-10

R. A. Shea

H5-52

J. E. Shapley

SO- 02

T. N. Shaw

$\mathrm{N} 1-83$

E. M. Sheen

S4-55

E. J. Shen

L7-05

S4-58

P. K. Shen

HO-39

A. T. Shook

S2-01

A. B. Sidpara

A2-91

S. G. Spencer

S. M. Stahl

E. G. Stephan

R1 -30

H5-31

A3-74

R. R. Stickney

R1-49

T. L. Strathman

SO- 14

J. N. Strode

RI -51

K. C. Strong

M. J. Sutey

R2-12

R1 -49

D. G. Sutherland

L4 -72

J. P. Summerhays

R2 - 88

L. M. Swanson

H5 -34

J. F. Thompson

H5-71

J. D. Thomson

R1 -30

S. R. Tifft

H6-26

J. A. Tilden (2)

L6- 10

H. Toffer

HO- 38

T. T. Tran

L7 -05

J. B. Truitt

H5-56

R. E. Vandercook

S6-07

R. J. Van Vleet

H5-32

J. A. Voogd

J. R. Weber

R4-03

G6-46

D. L. Wegener

R1 -62

R. K. Welty

R1 -80

G. T. Wells

H6-26

K. A. White

Rl-51

D. D. Wodrich

$B 1-59$

B. D. Zimmerman

LO-06 
WHC-EP-0182-57

Distribution - continued

$\begin{array}{ll}\text { Docket File (2) } & \mathrm{H} 5-36 \\ \text { 272-AW Shift Office } & \mathrm{S} 5-04 \\ \text { Central Files (2) } & \mathrm{L} 8-04 \\ \text { Tank Farms Info Center } & \mathrm{R} 1-28 \\ \text { Information Release } & \\ \text { Administration (3) } & \mathrm{R} 1-08 \\ \text { Env Data Mgmt Center } & \mathrm{H} 4-22\end{array}$

Distr-15 

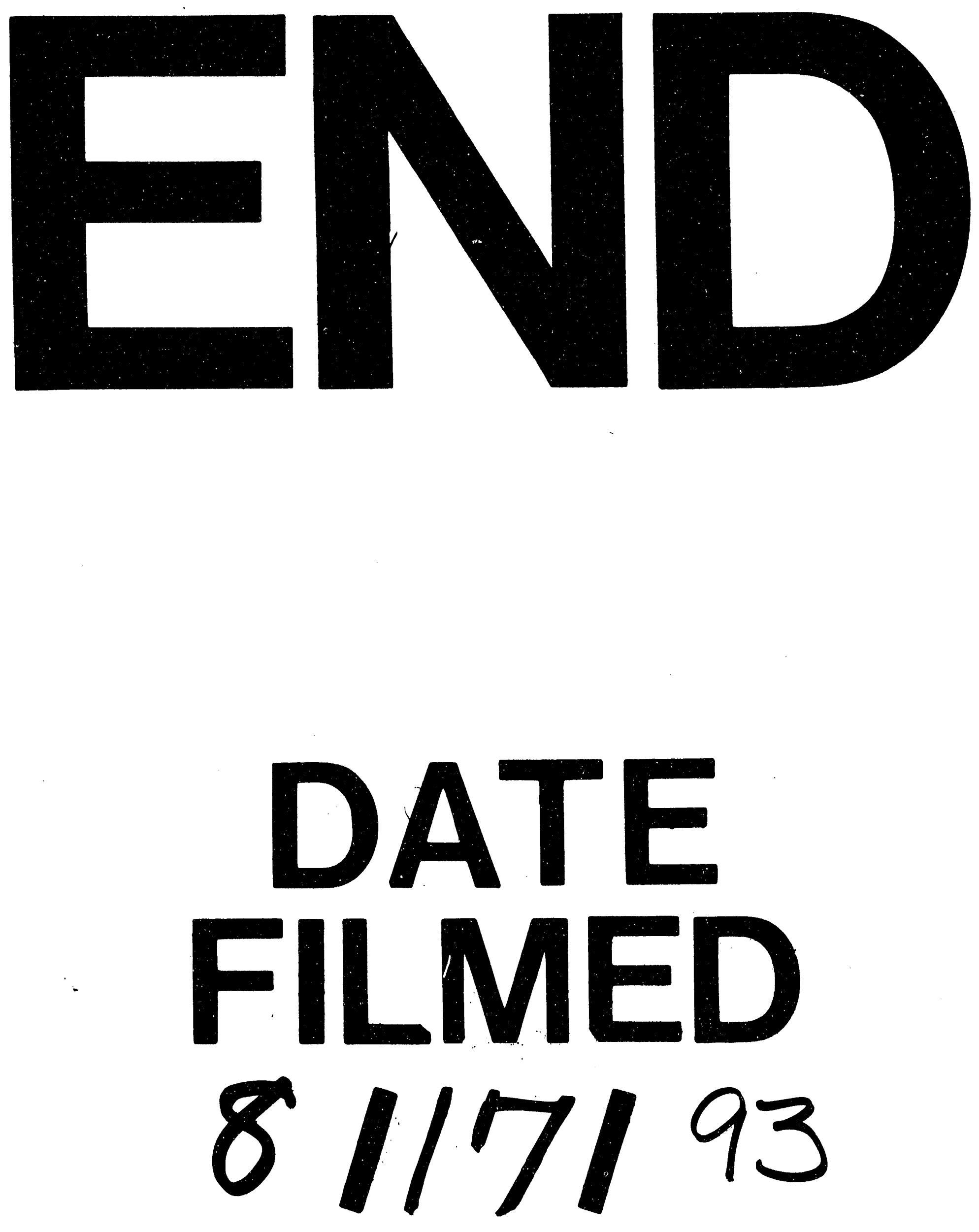

1 